\title{
The plant diversity in Bukit Timah Nature Reserve, Singapore
}

\author{
B.C. Ho ${ }^{1}$, H.K. Lua ${ }^{2}$, Bazilah Ibrahim ${ }^{1}$, R.S.W. Yeo ${ }^{1}$, P. Athen ${ }^{1}$, P.K.F. Leong ${ }^{1}$, Ali \\ Ibrahim $^{1}$, S.L. Koh ${ }^{1}$, Hassan Ibrahim ${ }^{3}$, S. Lindsay ${ }^{4}$, L.L. Chin ${ }^{1}$, W.W. Seah ${ }^{1} \&$ D.J. \\ Middleton ${ }^{{ }^{*}}$
}

${ }^{1}$ Singapore Botanic Gardens, National Parks Board,

1 Cluny Road, 259569 Singapore

*david_middleton@nparks.gov.sg

${ }^{2}$ National Biodiversity Centre, National Parks Board,

1 Cluny Road, 259569 Singapore

${ }^{3}$ International Biodiversity Conservation, National Parks Board,

1 Cluny Road, 259569 Singapore

${ }^{4}$ Native Plant Centre, Horticulture \& Community Gardening,

National Parks Board, Pasir Panjang Nursery,

100K Pasir Panjang Road, 118526 Singapore

\begin{abstract}
The plant diversity of Bukit Timah Nature Reserve (BTNR) is relatively well studied due to concerted effort over several decades, particularly as part of the worldwide system of ecological plots set up by the Center for Tropical Forest Science (CTFS), now called the Forest Global Earth Observatory. Publications arising from previous works have set baseline data for the species diversity, suggested that the forest resilience was greater than would be expected in such a small forest fragment, but that there was low recruitment of primary forest tree species into the secondary forest. In order to assess the overall vascular plant diversity, and to compare the diversity of the various forest types within BTNR to each other, 52 plots were set up, each $20 \times 5 \mathrm{~m}$, along nine different transects that covered the full range of topography and forest types, primary, old secondary and maturing secondary forests, within the reserve. The vascular plant diversity within each plot was recorded. In total, 1250 species in 148 families were recorded, including an additional 167 species newly listed for BTNR. The primary forest had the highest number of species not found in the other forest types. It nevertheless had a very large overlap with species in the old secondary forest but not with the maturing secondary forest.
\end{abstract}

Keywords. conservation, plots, primary forest, recruitment, secondary forest, transects

\section{Introduction}

The plant diversity and ecology of the Bukit Timah Nature Reserve (BTNR) is arguably better known than any other patch of forest in Singapore (Wong, 1987; Swan, 1988; Corlett, 1990; Corlett, 1995; Tan et al., 1995; Wee, 1995a, 1995b; Wee \& Haji Mohamed, 1995; LaFrankie et al., 1996; Lum et al., 2004; Turner \& Chua, 2011; Chua et al., 2013; Ngo et al., 2016), yet still yields species new to science (LeongŠkorničková \& Boyce, 2015), new records of species previously unknown in 
Singapore (Chen et al., 2018; Ho et al., 2018; Khoo et al., 2018; Lim et al., 2018), and rediscoveries of species thought to be extinct in Singapore (Ho et al., 2018). The botanical importance of this forest is also reflected in the type specimens of the 37 taxa and two hybrids that were first described from plants collected on Bukit Timah and because it is the only place in the world where Hanguana triangulata Škorničk. \& P.C.Boyce is known to occur (Leong-Škorničková \& Boyce, 2015; Niissalo \& Leong-Škorničková, 2017).

The 163 ha nature reserve consists of about 48 ha of unlogged primary forest and the rest is a patchwork of secondary forest, the oldest parts of which have been regenerating since the 1950s. The history of BTNR, leading to the vegetation zones seen today, is described by Davison \& Chew (2019). The primary forest patch has been classified as 'coastal hill dipterocarp forest' by a number of authors (Wong, 1987; Corlett, 1995; Symington et al., 2004) and forms the largest remaining patch of primary forest in Singapore, which, together with some patches in the Central Catchment, constitutes only $0.28 \%$ of the original forested area of Singapore (Yee et al., 2011).

In 1993, a plot of 2 ha $(200 \times 100 \mathrm{~m})$ within the primary forest was established by the Center for Tropical Forest Science (CTFS), currently known as Forest Global Earth Observatory (ForestGEO) (LaFrankie et al., 2005). This 2-ha plot was re-censused in 1995, 2003, 2008 and 2012 (Ngo et al., 2016). An additional plot of 2 ha was set up in mature secondary forest adjacent to the primary forest plot in 2003 . Between 2005 and 2008, the same team extended the survey to the entire BTNR to include all trees with at least $30 \mathrm{~cm}$ dbh (see Khoo et al., 2018). These are part of a much wider system of plots in various forest types around the world to study forest ecology and dynamics by the CTFS-ForestGEO. On Bukit Timah, it has been possible to study dynamics within the 2-ha plots by measuring all trees in various size classes of at least $1 \mathrm{~cm} \mathrm{dbh}$, allowing the calculation of basal area changes, and increases and reductions of species over time. Due to the small size of the forest on Bukit Timah, one would expect there to be species loss, higher numbers of invasive species, higher numbers of pioneer tree species, lower numbers of animal dispersed species, and a reduction of overall biomass over time, features typically associated with forest fragmentation (see Ngo et al., 2016). However, Ngo et al. (2016) reported that the tree diversity and forest structure of Bukit Timah was proving to be remarkably resilient and that there was no evidence of collapse or dramatic transformation. They concluded that observed changes in relative tree species diversity since 1993 had largely been driven by the effects of droughts in 1997 and 2009 rather than due to the effects of forest fragmentation.

The loss of individuals and taxa due to forest fragmentation is not spread evenly between the different plant life-forms (trees, lianas, herbs etc.) in a forest (Pasion et al., 2018). Niissalo et al. (2017) noted that in forests suffering species loss due to fragmentation, trees are the least threatened of life forms, whilst epiphytes are the most threatened with an estimated $63 \%$ of species recorded for Singapore now extinct. They also observed that despite the apparent resilience of plant species overall in Singapore, the very high percentage of threatened species in Singapore (Davison et al., 2008) suggests that extinction debt, delayed yet anticipated extinctions due to past events, 
still exceeds the actual extinctions. They found that even though the secondary forest surrounding much of the primary forest in Singapore has became mature, such that it would appear that the forest has recovered well, the primary forest herbs they studied were nevertheless confined to the primary forest fragments (Niissalo et al., 2017). Chua et al. (2013) also reported that recruitment of primary forest tree species into an almost contiguous plot of mature secondary forest (after 56 years of growth) on Bukit Timah was extremely low. They did note, however, that there was no appreciable difference in soil quality between the primary and old secondary forests they studied, thereby reducing at least one barrier to future recruitment into the secondary forest. Despite appearances, the primary forests in Singapore remain very fragmented.

Turner \& Chua (2011) published a checklist of the vascular plants of Bukit Timah, along with voucher information and/or references to back up the record for most species. They listed 1047 species in all, providing a valuable baseline for further survey work within BTNR.

With this background, this study sets out to survey the plant diversity along transects in both primary and secondary forests. In doing so, we shall compare the species diversities between the transects and compare the various forest types present. We shall also compare the overall species diversity found in BTNR to the species list of Turner \& Chua (2011), updating the taxonomy and adding to the list as necessary (Appendix I).

\section{Materials and Methods}

The comprehensive biodiversity survey of BTNR has been introduced by Chan \& Davison (2019a). To explore the diversity of the different forest types in BTNR, nine transects were established. These transects covered the primary (P: 27 plots), old secondary (O: 20 plots) and maturing secondary (M: 5 plots) forest types in BTNR and were set up along existing trails and stream courses (see Fig. 1). The background and criteria to distinguish these forest types are described by Chan \& Davison (2019a), accounting for deviations from ideal botanical sampling methodology, and further details of the floristics within each type are given by Chan \& Davison (2019b). The forest types in each of the nine transects are as follows:

1. South View Path (SVP) - P, O

2. Taban Stream (TBS) - O

3. Cave Path-Catchment Path (CCP) - O

4. Tiup Tiup Stream (TTS) - P

5. Jungle Fall Path (JFP) - P, O

6. Jungle Fall Stream (JFS) - P, O

7. Main Road Path (MRP) - P

8. Fern Valley Stream (FVS) - P, O

9. Lasia Track (LST) - M 
Description of the Transects

1. South View Path (SVP01-SVP06) - The transect (6 plots) follows the trail from near the foot of BTNR, rising along the side of the hill overlooking Taban Valley until reaching a high point at South View Hut. The vegetation is largely old secondary forest with the occasional remnant primary forest tree exemplified by the large buttressed Parishia insignis Hook.f. between plots SVP01 and SVP02. The main canopy is at 20 to $30 \mathrm{~m}$ high and there is significant regeneration of large saplings to pole-sized trees, dominated by Streblus elongatus (Miq.) Corner and Palaquium gutta (Hook.) Baill. The forest becomes more or less of a primary nature near the junction with the Taban Loop trail with large dipterocarp trees on either side of the trail, before becoming old secondary forest again towards South View Hut.

2. Taban Stream (TBS07-TBS09) - The Taban Stream transect ( 3 plots) begins near the start of Taban Loop, although there is no obvious surface water on the sloping forest floor. Two or three old terraces that were cut into the higher slope are still present. Large granite boulders and weathered rocks occur at mid-transect where surface water begins to appear from small tributaries. The forest at the beginning is degraded primary forest with several large mature trees, before a transition into old secondary forest with saplings and young sub-adult trees in the understorey dominated by Streblus elongatus and Palaquium gutta where the stream flows under a bridge of the Taban Loop trail. The old secondary forest continues after the wooden bridge where evidence of past human impact is seen in old wells and small open patches of introduced shrubbery. Nonetheless, the forest structure remains relatively intact.

3. Cave Path-Catchment Path (CCP10-CCP17) - This transect (8 plots) begins along the final stretch of Cave Path trail where a small stream trickles down the slope onto a cement platform beside the trail. The trail hugs the side of the hill before joining the Catchment Path that descends from the hill slope. The old secondary forest along Cave Path is partially open and the crowns of old trees (e.g. Campnosperma auriculatum Hook.f. and Streblus elongatus) form the mid-canopy layer. Small patches of Dicranopteris sp. occur in forest gaps along the trail. The forest sharply changes into degraded primary forest with the occurrence of large Castanopsis lucida (Nees) Soepadmo and Shorea curtisii Dyer ex King trees just before the junction with the Catchment Path. The transect continues downwards along the Catchment Path through old secondary forest, with a dense understorey of shrubs and saplings and the occasional relict primary forest tree, before gradually flattening out at the foot of BTNR within mature secondary forest consisting of pioneer tree species with a low canopy height of up to 20 metres plus a few larger trees.

4. Tiup Tiup Stream (TTS18-TTS21) — The transect (4 plots) begins from the Ngadiman Bridge over a normally dry stream bed along the now-closed Tiup Tiup Path. The stream bed descends steeply down a valley, with no surface water present until midway down the transect. From this point, a flowing stream with a sandy-muddy bottom appears where the valley floor widens slightly from plot TTS19. 


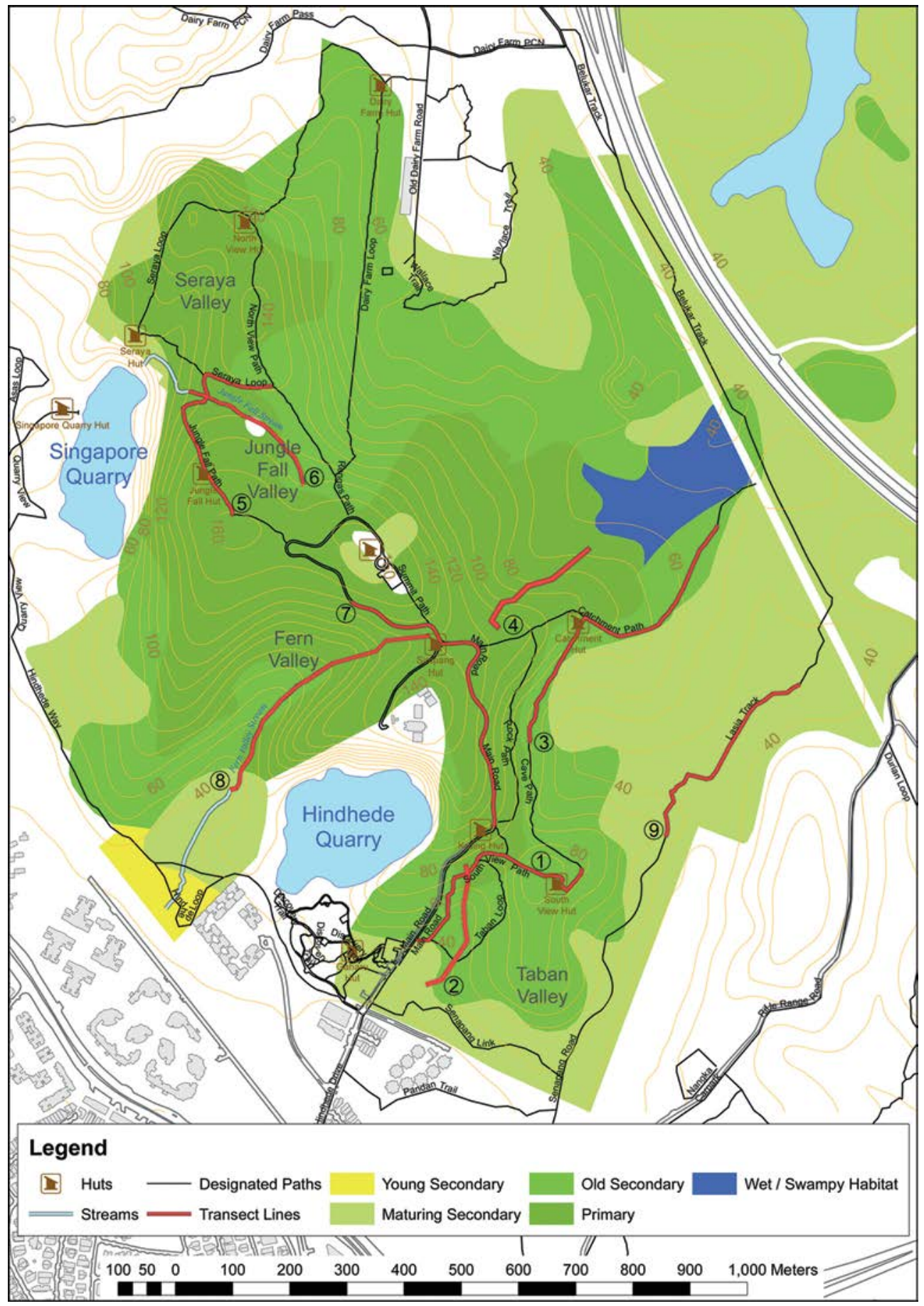

Fig. 1. Map of Bukit Timah Nature Reserve delineating the different forest types and the locations of the nine selected transects studied: numbered 1 to 9 (Source: NParks). 
Rocks and boulders line the valley bottom, where the stream occasionally disappears and re-emerges, until the valley flattens out towards the end of the transect. The transect mostly runs through primary forest with emergent trees up to $50 \mathrm{~m}$ tall along the valley slopes. The canopy along the stream is somewhat lower and more open with a denser understorey due to the increased light levels. Beyond plot TTS19, the forest becomes a somewhat mature secondary forest with large Campnosperma auriculatum and Ixonanthes reticulata Jack trees forming part of the main canopy.

5. Jungle Fall Path (JFP22-JFP27) - This transect (6 plots) is along a trail that traverses the valley of Jungle Fall Stream, beginning from the end of the western spur of the main summit where the path splits from the disused Hampstead Path. From the top of the valley, the trail descends steeply to the bottom of the valley through primary forest and passes close to the quarry cliff edge to the west. At the valley bottom, the trail crosses over Jungle Fall Stream flowing westwards towards Singapore Quarry cliff. The trail continues up the steep valley slope before becoming more gentle along the eastern side of the valley, ending at the junction with North View Path. The transect is contained almost entirely entirely within the primary forest ofthe trail, dominated by Shorea curtisii, with the main canopy generally at 30-40 m high with the occasional emergent reaching 50-60 m.

6. Jungle Fall Stream (JFS28-JFS32) - The transect (5 plots) begins at the source of the Jungle Fall Stream, marked by an old well at the base of a steep slope northwest of the summit. The V-shaped valley is steep-sided and was likely formed over a long period by the stream. The forest at the beginning of the transect comprises primary forest to the west, with a canopy of about $40 \mathrm{~m}$ high, and a denser old secondary forest with a lower canopy to the east. The rattan-dominated old secondary forest covers one-third of the transect along the valley before gradually merging into primary forest downstream. Two small old concrete dams along the lower stream remain as reminders of past disturbance, before the stream passes under the Jungle Fall Path, continuing about $50 \mathrm{~m}$ further and draining over the steep cliffs of the Singapore Quarry.

7. Main Road Path (MRP33-MRP40) - This transect (8 plots) follows BTNR's oldest path, which is paved and suitable for, albeit restricted, vehicular access to the summit. The transect begins just below the slope of the summit to the north and winds downhill past Fern Valley to the south, then past the junction with Quarry Path and continues down the side of the steep hill slope, ending just before Keruing Hut. The transect is entirely within primary forest dominated by Shorea curtisii, the main canopy at $30-40 \mathrm{~m}$ high and occasionally with emergents of up to $50 \mathrm{~m}$ high. The main canopy comprises a diverse range of trees including the families Burseraceae, Fabaceae, Fagaceae, Malvaceae and other Dipterocarpaceae. There are gaps from old and more recent tree falls, mainly at the beginning of the transect where the forest is more open than in the lower half of the transect after the junction with Quarry Path. The understorey is generally sparse except where there are old gaps with an increase in sapling density and vegetative regrowth. 
8. Fern Valley Stream (FVS41-FVS47) — This transect (7 plots) begins near the start of Fern Valley not far from the junction of MRP and the Quarry Path. The transect follows the valley. The valley descends rather rapidly with steep sides with an area of old secondary forest to the northeast in the upper third of the transect. Mid-way through the valley, the transect enters a boulder-strewn area which continues for most of the remainder of the transect before abruptly flattening out near the base of BTNR. The stream appears from under the boulders towards the end of the valley bottom where it forms a small constant flow through the narrow flat area. The forest here is almost entirely pristine primary forest with visibly more bryophytes and ferns along the damp and humid boulder field. The exceptions are the old secondary forest near the beginning, and the maturing secondary forest at the end of the transect which is partly within the final plot FVS47 with the pioneer Campnosperma auriculatum as the main canopy tree and remnants of ornamental plants from former village cultivation along the plain.

9. Lasia Track (LST48-LST52) - This trail (5 plots) passes entirely through maturing secondary forest that has established on former rural village areas, abandoned possibly in the 1970s. This forest is dominated by native pioneer trees including Campnosperma auriculatum, Claoxylon indicum (Reinw. ex Blume) Hassk., Ficus vasculosa Wall. ex Miq., Macaranga gigantea (Rchb.f. \& Zoll.) Müll.Arg. and Prunus polystachya (Hook.f.) Kalkman, as well as other remnants of fruit trees cultivated in the past by villagers. The transect begins near the foot of BTNR's slopes and then follows the Lasia Stream which winds along a low-lying area to the Rifle Range forest. The topography is generally flat with gentle undulations from the lower slopes of BTNR to the northwest. The forest is younger than the adjacent old secondary forest of Taban Valley and the Cave Path/Catchment Path and does not abut any primary forest area.

\section{Sampling Methods}

Every $50 \mathrm{~m}$ along each transect, a plot of 0.01 ha $(20 \times 5 \mathrm{~m})$ was established. The number of plots per transect ranged from three to eight (detailed above), and transect length ranged from $200 \mathrm{~m}$ to $570 \mathrm{~m}$. The plots were sequentially placed on alternate sides of the transect (see Fig. 2) until all transects were completely sampled. The plots were numbered sequentially regardless of a change in transect.

The surveys were carried out from May 2015 to July 2017. Each plot was surveyed completely for all plants before moving on to the next plot. A brief description of the forest and habitat was recorded at the commencement of each plot survey.

\section{Identification}

In this study, all vascular plants, including trees, shrubs, climbers, herbs, epiphytes, ferns and lycophytes, found within each plot were recorded. Any species that was readily identifiable in the field was recorded in a notebook but not collected unless fertile. Samples of individuals that required further verification and identification were collected for later study in the Herbarium of Singapore Botanic Gardens. All plants were named to the best of our knowledge by the authors of this paper, by the 
taxonomists of Singapore Botanic Gardens, by other NParks colleagues and by visiting specialists. The correct name was established by reference to available literature and by matching with authoritatively named herbarium specimens. Representative vouchers are deposited in the Herbarium of Singapore Botanic Gardens (SING).

\section{Statistical Analysis}

The dissimilarity of each of the 52 plots to every other plot using non-metric multidimensional scaling (NMDS) was calculated. The NMDS was conducted using the Bray-Curtis dissimilarity index at the species level using presence-absence data, implemented with the vegan package. A stress value of 0.20 and below was deemed as acceptable for interpretation of the ordination on a two-dimensional plot (Clarke, 1993). A graphical representation of the NMDS plot was performed using the ggplot2 package.

All statistical analyses were performed using R version 3.4.3 (R Core Team, 2016) and the package vegan 2.4-6 (Oksanen et al., 2018).

\section{Results}

A total of 52 plots covering an area of 0.52 ha of forest vegetation was surveyed between May 2015 and July 2017. The Species Accumulation Curve does not reach a plateau for the 52 plots (Fig. 3).

The total number of species recorded in this study was 839 in 126 families, including 42 species that were either juveniles or sterile that could not be named but were included in the NMDS analyses as unique species (See Appendix I). Of the species found, four were lycophytes, 51 were ferns and two were gnetophytes, while the rest of the 782 species were angiosperms. Thirteen species were confidently identified as representing either new species records for Singapore (Passiflora quadriglandulosa Rodschied, Scindapsus lucens Bogner \& P.C.Boyce and Tectaria nayarii Mazumdar) or rediscoveries of species listed in Davison et al. (2008) as nationally extinct (Aglaia palembanica Miq., Bolbitis sinuata (C.Presl) Hennipman, Calamus ornatus Blume., Claoxylon longifolium (Blume) Endl. ex Hassk., Dapania racemosa Korth., Dioscorea kingii R.Knuth, Ficus rosulata C.C.Berg, Lasianthus reticulatus Blume, Ryparosa hullettii King and Senegalia kekapur (I.C.Nielsen) Maslin, Seigler \& Ebinger). These have been reported separately (Ho et al., 2018).

Turner \& Chua (2011) compiled a list of 1047 species of vascular plants from BTNR. When those lacking vouchers for verification are excluded, along with nomenclatural updates from recent revisions and following APG IV (2016), 959 species in 137 families remain. An additional 124 species in 55 families (including seven new families: Amaranthaceae, Capparaceae, Lentibulariaceae, Linderniaceae, Malpighiaceae, Muntingiaceae and Plantaginaceae) have been reported elsewhere for BTNR (King, 1890; Ridley, 1900; 1901; Keng, 1990; van Steenis, 1977; 


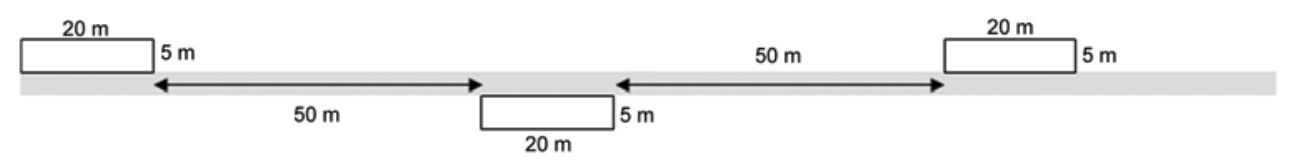

Fig. 2. Sampling method.

Ali Ibrahim et al., 1997; Keng et al., 1998; Ng et al., 2014; Niissalo et al., 2014; de Kok, 2015; Leong-Škorničková \& Boyce, 2015; Niissalo et al., 2016; Leong et al., 2017; Niissalo et al., 2017; Ho et al., 2018; Khoo et al., 2018; Leong et al., 2018; Lim et al., 2018; Niissalo et al., 2018; Rodda \& Lai, 2018; Turner \& Kumar, 2018; Ascher et al., 2019), including some published before 2011 that were omitted by Turner \& Chua (2011) (see Appendix II). In our study, an additional 167 species and four families (Cardiopteridaceae, Flagellariaceae, Heliconiaceae and Musaceae) that were not previously listed for BTNR have been recorded from at least one of the 52 plots (Appendix III). Of the species previously reported for BTNR, 454 were not found in any of our plots, although 108 of these species are already believed to be nationally extinct (Davison et al., 2008; Chong et al., 2009) and, of the rest, some were collected outside the sampling plots or are known to be present elsewhere in BTNR. When all records are combined, a total of 1250 species of vascular plant in 148 families have now been recorded in BTNR, excluding 43 plants that cannot be adequately named (Table 1). The current study captured $64 \%$ of these 1250 species, or $70 \%$ of the nationally extant 1142 species in BTNR. All 55 species of ferns and lycophytes captured in the plots were already known from BTNR except for Palhinhaea cernua (L.) Franco \& Vasc. However, 57 species, or 51\%, of the ferns and lycophytes previously reported for BTNR, including nine nationally extinct species, were not found in this study. This is much higher than for flowering plants where the comparable figure is $35 \%$ of the species.

The number of species found within and shared between each forest type, namely primary forest $(\mathrm{P})$, old secondary forest $(\mathrm{O})$ and maturing secondary forest (M), was also calculated (Fig. 4). In the primary forest, 227 unique species out of the total 839 identified species were found, while for the old secondary and maturing secondary forests the figures were 124 and 29 unique species, respectively. Figure 4 shows the numbers of species shared between any two forest types plus the 68 species that were found in all three forest types. It is noticeable that these 68 species include only two species of Lauraceae, which were otherwise diverse in both the primary (20 species) and old secondary forests (15 species). Most members of the Lauraceae in our plots occur uniquely in either primary or old secondary forest. They could be a good indicator for these forest types. Figure 4 shows a relatively high number of species $(354+68=422$ species $)$ that were found in both primary and old secondary forests. The maturing secondary forest had the highest number of non-native species (27) compared to old secondary and primary forests (13 and 12, respectively). Clidemia hirta (L.) D.Don and Ficus punctata Thunb. were the only non-native species found in all three forest types. Although maturing secondary forest was 


\section{Species Accumulation Curve}

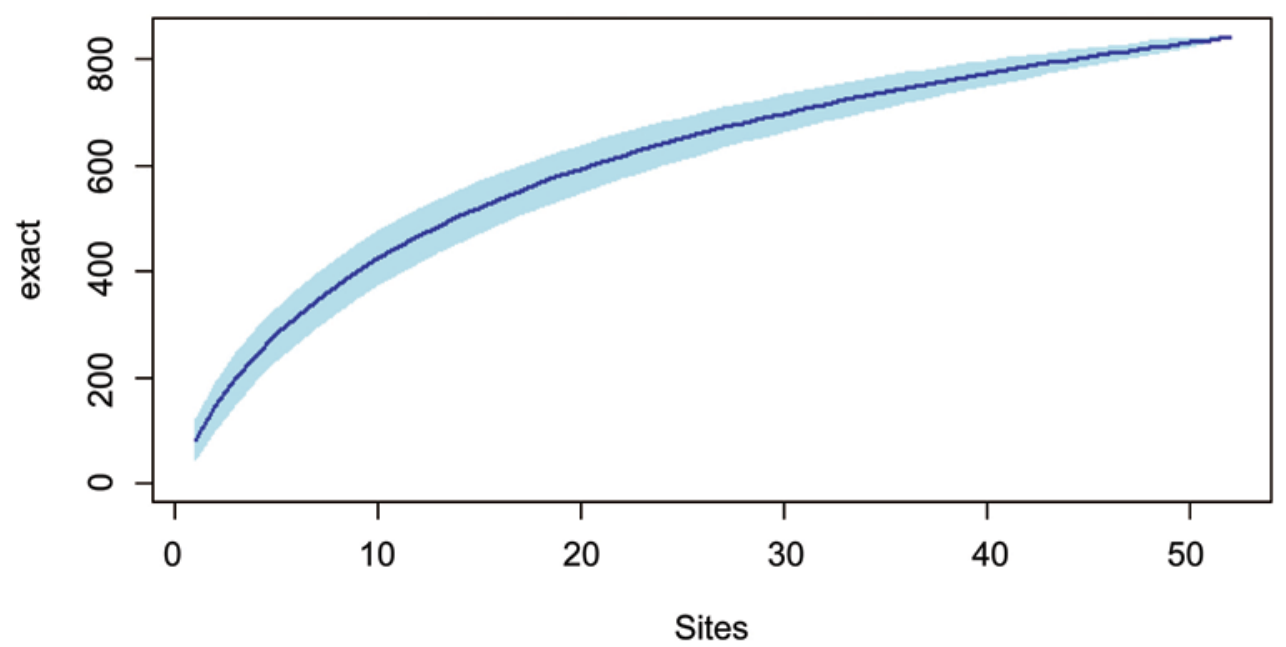

Fig. 3. The Species Accumulation Curve (SAC) presents the rate at which plant species were found within the current study. The mean SAC and its standard deviation are derived from random permutations of the data, or subsampling without replacement (Gotelli \& Colwell, 2001).

under-represented in our study, the limited plot numbers already show observable differences in species composition and structure.

Dipterocarpaceae, an important component of rain forests in Southeast Asia, were represented by 15 species in our plots, missing three species that were reported by Turner $\&$ Chua (2011). Of these 15 species, 13 and 6 of them respectively were found within the primary and old secondary forests, while none was recorded for the maturing secondary forest.

Among the nine transects, MRP had the highest species richness (334 spp.), followed by JFS (322 spp.), while LST had the lowest (134 spp.) (see Table 2). TTS, JFS and MRP, had the highest number of Dipterocarpaceae species, at seven apiece. Of the 839 species recorded in this study, 39.1\% were recorded from only one of the transects. Transect FVS, which consists of a mixture of $\mathrm{P}$ and $\mathrm{O}$ forest types, had the highest number of species only known from that transect at $22.3 \%$, followed by LST ( $\mathrm{M}$ forest type) at $20.9 \%$. On the other hand, JFP (P and $\mathrm{O}$ forest types) had the lowest number of unique species amongst the sampled plots (Table 2). Almost $60 \%$ of the species captured in our study occurred in only one or two transects, whilst fewer than $20 \%$ of the species were found in five or more transects (Table 3 ).

Figure 5 shows the non-metric multidimensional scaling (NMDS) that was conducted using the Bray-Curtis dissimilarity index at the species level. Using this method, the plots that had more similar species composition, using presence-absence data, are closer together and, conversely, those that were more dissimilar are further 
Table 1. The number of families and species of vascular plants for BTNR from past and present records. Family classification follows APG IV (including retroactively for older publications). Species numbers exclude all dubious records. The numbers in square brackets denote additional species recorded from specimens that cannot be adequately named but represent distinct morphospecies. The additional published records include those omitted by Turner \& Chua (2011) or published afterwards (see Appendix II for details). The new records in this study include those species not previously reported for BTNR in any published studies (see Appendix III for details).

\begin{tabular}{lllll}
\hline & $\begin{array}{l}\text { Turner \& Chua } \\
\text { (2011) }\end{array}$ & $\begin{array}{l}\text { Additional pub- } \\
\text { lished records }\end{array}$ & $\begin{array}{l}\text { New records in } \\
\text { this study }\end{array}$ & Total \\
\hline Families & 137 & 7 & 4 & 148 \\
Species & $959[1]$ & 124 & $167[42]$ & $1250[43]$ \\
\hline
\end{tabular}

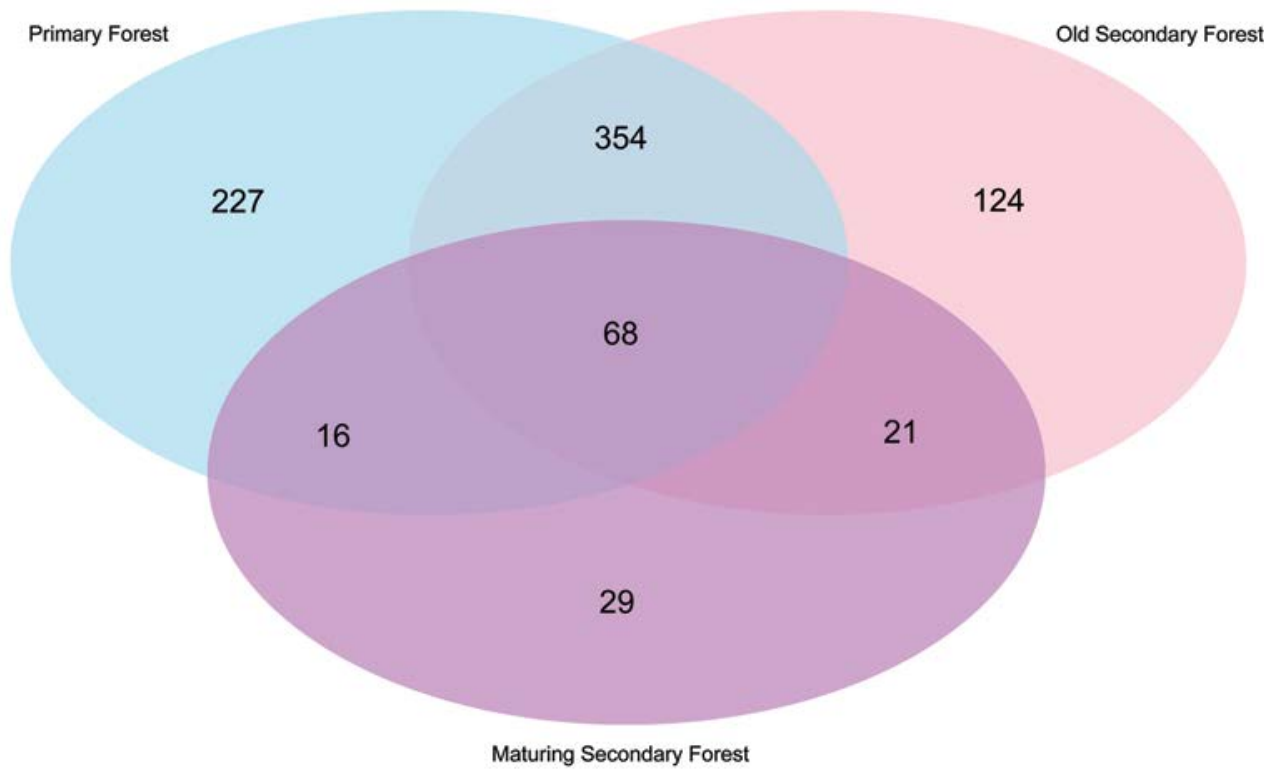

Fig. 4. Venn diagram of the number of plant species found only in each forest type and number of plant species encountered in two or more forest types, indicated by the overlaps in the Venn diagram. A total of 839 species were sampled from the 52 plots, including 27 plots in primary forest, 20 plots in old secondary forest and 5 plots in maturing secondary forest.

apart. The results are rather inconclusive except that the plots from LST (plots 48 to 52), all of which were in maturing secondary forest, were distinct from the others. In addition, FVS plots 44, 45 and 46, all of which were from primary forest, were also rather distinct from the rest of the plots but were more similar to the old secondary forest plots from FVS than to all other primary forest plots except for TTS21. 
Table 2. Summary of the nine transects to show the number of plots sampled for each transect, the forest types ( $\mathrm{P}=$ Primary; $\mathrm{O}=$ Old Secondary; $\mathrm{M}=$ Maturing Secondary), the species richness, and the number of species only known from one transect in the current study. In total, 839 species in 127 families were captured.

\begin{tabular}{llllllc}
\hline Transect name & Abbrev. & $\begin{array}{l}\text { Sampled } \\
\text { Plot }\end{array}$ & $\begin{array}{l}\text { Forest } \\
\text { type }^{1}\end{array}$ & $\begin{array}{c}\text { Spp. } \\
\text { Richness }\end{array}$ & $\begin{array}{c}\text { Spp. in one transect } \\
\text { no. }\end{array}$ & $\begin{array}{l}\text { \% } \\
\text { South View Path }\end{array}$ \\
Taban Stream & SVP & 6 & P/O & 284 & 33 & 11.6 \\
Cave Path-Catchment & TBS & 3 & O & 193 & 26 & 13.5 \\
Path & CCP & 8 & O & 268 & 30 & 11.2 \\
Tiup Tiup Stream & TTS & 4 & P & 228 & 28 & 12.3 \\
Jungle Fall Path & JFP & 6 & P/O & 288 & 25 & 8.7 \\
Jungle Fall Stream & JFS & 5 & P/O & 322 & 51 & 15.8 \\
Main Road Path & MRP & 8 & P & 334 & 42 & 12.6 \\
Fern Valley Stream & FVS & 7 & P/O & 291 & 65 & 22.3 \\
Lasia Track & LST & 5 & M & 134 & 28 & 20.9 \\
\hline & Total & 52 & & 839 & 328 & 39.1 \\
\hline
\end{tabular}

Table 3. The number of transects in which each of the total of 839 plant species was recorded, from 1 where a species was recorded along only one transect up to a maximum of 9 where a species occurs along all of the transects.

\begin{tabular}{llllllllllll}
\hline $\begin{array}{l}\text { Total number of } \\
\text { transects in which } \\
\text { given no. of plant } \\
\text { species occur }\end{array}$ & $\mathbf{1}$ & $\mathbf{2}$ & $\mathbf{3}$ & $\mathbf{4}$ & $\mathbf{5}$ & $\mathbf{6}$ & $\mathbf{7}$ & $\mathbf{8}$ & $\mathbf{9}$ & all \\
\hline No. species & 328 & 165 & 102 & 78 & 60 & 33 & 30 & 30 & 13 & 839 \\
$\%$ species & 39.1 & 19.6 & 12.2 & 9.3 & 7.2 & 3.9 & 3.6 & 3.6 & 1.5 & 100 \\
\hline
\end{tabular}

\section{Discussion}

Appendix I presents an updated checklist of the vascular plant species recorded from BTNR, 1250 species in 148 families. From Chong et al. (2009), and records published since, there are estimated to be around 2750 species of native, naturalised and casual vascular plant species in Singapore which means that the plant diversity of BTNR accounts for around $45 \%$ of total vascular plant diversity in Singapore. When the exotic species are excluded (including species for which it is currently unknown whether they are exotic or not), then the 1208 native species in BTNR account for 


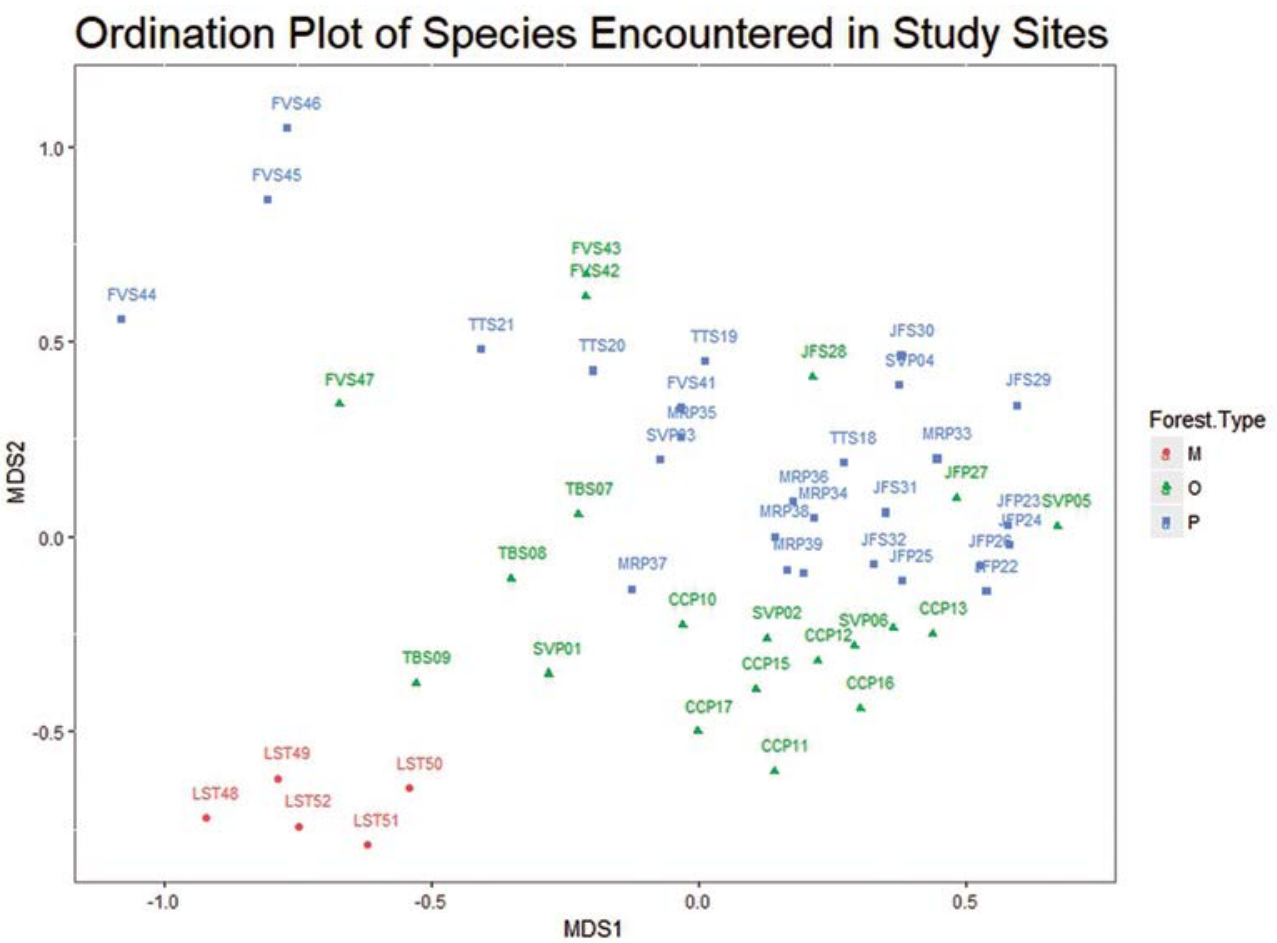

Fig. 5. Non-metric multidimensional scaling (NMDS) ordination plot of Bray-Curtis community dissimilarities based on the species of plants found in the 52 plots (2D stress value $=0.18$ ). The shorter the distance between two samples, the higher the similarity between these plots. The 52 sampled plots are labelled according to the transect (see Materials and methods) and the plot number; the colour and symbol shape refers to the forest types ( $\mathrm{P}=$ Primary: 27 plots; $\mathrm{O}=$ Old Secondary: 20 plots; $\mathrm{M}=$ Maturing Secondary: 5 plots).

over half, about 55\%, of the estimated 2215 native species in Singapore. When one considers that BTNR has half of Singapore's native plant species in around just $0.2 \%$ of Singapore's land surface, it highlights just how remarkable the nature reserve is. It should also be noted that 167 species ( 80 tree species, 61 climbers, 12 shrubs, 13 herbs and one epiphyte) were found in this study that were previously not known to occur in BTNR, with the possibility that more could be found with further surveys. However, 454 species previously recorded from BTNR were not found in this study of which 108 are already recorded as likely to be nationally extinct in Singapore (Davison et al., 2008; Chong et al., 2009). Of the remainder, their conservation status in BTNR will need to be further ascertained (Turner \& Corlett, 1996) as this survey is not a complete census of the forest as shown in Fig. 3. The Species Accumulation Curve (Fig. 3) does not reach a plateau, indicating that if there had been additional plots, including plots further into the forest away from the trails, further diversity would likely have been found. Appendix I shows which species were recorded by Turner \& Chua (2011) but not found in our plots and these include 57 species of lycophytes and ferns and large 
numbers of angiosperm herbs, climbers and understorey trees, but relatively fewer large forest trees. To some extent, this disparity may be due to the difficulty of finding and collecting epiphytes but may also be due to the likelihood that more of these life-forms really have been lost compared to the large forest trees (Turner, 1996).

A number of studies in Singapore have commented on how little recruitment there is of primary forest species into secondary forest (see Chua et al., 2013; Niissalo et al., 2017). In our study, we recorded 227 species that were only found in the primary forest but a further 354 species shared with the old secondary forest, 68 species found in all three forest types and 16 shared between the primary forest and the maturing secondary forest (Fig. 4). This last overlap is perhaps the most surprising but is possibly an artefact of the small number of individuals of the species concerned that they were not also found in the old secondary forest plots. Overall, however, the large overlap in species between the primary and old secondary forests suggests that there is recruitment into the secondary forest although the recruitment is low for iconic primary forest families such as in the Dipterocarpaceae and Zingiberaceae. Chua et al. (2013) noted a rapid drop in recruitment of primary forest species into secondary forest with increasing distance from the primary but many of our transects in old secondary forest are in close proximity to seed sources and consequently show signs of recolonisation. Dipterocarpaceae were entirely absent from the maturing secondary forest although no parts of this forest were contiguous with the primary forest. Also, note that the sampling in the maturing secondary forest is lower than in the other forest types (Table 2). Burseraceae, Cornaceae, Ebenaceae, Fagaceae, Lauraceae, Myristicaceae and Polygalaceae are also absent from the maturing secondary forest but all have a large overlap in species between the primary and old secondary forest suggesting a better rate of recruitment than for Dipterocarpaceae. A detailed study over time of the relative speed of recruitment of primary forest species into secondary forest could be conducted by re-surveying these same plots at regular intervals of say 10 years.

The 29 species only recorded from the maturing secondary forest are primarily remnant cultivated plants from earlier settlements and/or naturalising exotic herbaceous or shrubby species which, fortunately, appear not to be able to invade or survive in old secondary and primary forest. There were also 11 non-native species found in the primary forest, particularly along MRP, some of which are likely to be the result of deliberate plantings or due to unintended introduction by visitors or animals in the past, such as Elaeis guineensis Jacq., Hevea brasiliensis (Willd. ex A.Juss.) Müll.Arg. and Mangifera indica L.

The ordination study of the plots (Fig. 5) was rather inconclusive except for the distinction of the plots within the maturing secondary forest (LST48-LST52) compared to those in the primary and old secondary forests. This is rather unsurprising and corresponds to the much lower overlap in taxa between the maturing secondary forest and the other two forest types compared to the overlap between the primary and old secondary forest types in Fig. 4. The LST transect is in the youngest forest in BTNR and still shows its recent land-use history. The only other distinctive grouping is of FVS44-FVS46, all of which are primary forest plots. These plots have a particularly large number of species only found along this transect (and, being on the 
same side of one ridge, subject to similar environmental and dispersal influences), such that these primary forest plots are more similar to several old secondary forest plots along the same transect than they are to most primary forest plots elsewhere in BTNR. They come next closest to the plots along the TTS transect which is also notable for the large number of species only found along this transect. The large number of species only known from one transect (Table 2), particularly from FVS and TTS, may be an artefact of the sampling method but could also be indicative of these species being found in very low numbers in BTNR or otherwise very locally restricted for poorly understood reasons. This in turn has serious consequences for conservation policy and species recovery programmes, particularly to examine to what extent such low populations of species may still be reproductively viable (Niissalo et al., 2017).

\section{Conclusions}

Although the Bukit Timah Nature Reserve has long been known for high vascular plant diversity, we have found that the diversity of the reserve is even higher than previously known. At the same time, however, we have not found large numbers of species previously collected in BTNR and many of the extant species are only known from very small numbers of individuals. Although there remains a large number of species only found in the primary forest, recruitment of species from the primary forest into the old secondary forest is higher than previous studies have suggested.

ACKNOWLEDGEMENTS. We thank the following people: for administrative support, Adrian Loo, Cheryl Chia and Chew Ping Ting; for the field work, Saifuddin Suran, Hadzlinda Samri, Sunia Teo, Lam Hui Ju, Carmen Puglisi, Daniel Thomas, Matti Niissalo, Serena Lee, Reuben Lim, Cherish Yong, Low Wanting, Nurul Huda, Yeoh Yi Shuen, Amanda Yap, Lim Weihao and Mohd Farez Alias; for the analyses using R, Alex Yee and Prakki Sai Rama Sridatta; and for the Plant identifications, Jana Leong-Škorničková (Zingiberales, Hanguana), Daniel Thomas (Annonaceae), Willem de Wilde and Brigitta de Wilde-Duyfjes (Myristicaceae), and Wong Khoon Meng (Rubiaceae). We also thank our interns who assisted in fieldwork and specimen processing: Carmen Yuen, Nadya Hamdan, Tok Yin Xin, Faris Lim, Syahirul Fauzan Razali, Wong Shi Hong, Hew Ailin, Chloe Oon and Isaac Khor.

\section{References}

Ali Ibrahim, Chew, P.T., Sidek Kiah, H. \& Lai, J.T.K. (1997). New records of plant species from Singapore. Gard. Bull. Singapore 49: 49-54.

APG IV (2016). An update of the Angiosperm Phylogeny Group classification for the orders and families of flowering plants: APG IV. Bot. J. Linn. Soc. 181: 1-20.

Ascher, J.S., Soh, Z.W.W., Ho, B.M., Lee, R.Y.Y., Leong, A.Q.E., Chui, S.X., Lai, J.J.L., Lee, J.X.Q., M.-S. Foo \& Soh, E.J.Y. (2019). Bees of the Bukit Timah Nature Reserve and vicinity. Gard. Bull. Singapore 71 (Suppl. 1): 245-271. 
Chan, L. \& Davison, G.W.H. (2019a). Introduction to the Comprehensive Biodiversity Survey of Bukit Timah Nature Reserve, Singapore, 2014-2018. Gard. Bull. Singapore 71 (Suppl. 1): 3-17.

Chan, L. \& Davison, G.W.H. (2019b). Synthesis of results from the Comprehensive Biodiversity Survey of Bukit Timah Nature Reserve, Singapore, with recommendations for management. Gard. Bull. Singapore 71 (Suppl. 1): 583-610.

Chen, J., Turner, I.M., Saunders, R.M.K. \& Thomas, D.C. (2018). Artabotrys scortechinii (Annonaceae): an augmented species description and a new record for Singapore. Gard. Bull. Singapore 70: 3-8.

Chong, K.Y., Tan, H.T.W. \& Corlett, R.T. (2009). A Checklist of the Total Vascular Plant Flora of Singapore: Native, Naturalised and Cultivated Species. Singapore: Raffles Museum of Biodiversity Research.

Chua, S.C., Ramage, B.S., Ngo, K.M., Potts, M.D., \& Lum, S.K.Y. (2013). Slow recovery of a secondary tropical forest in Southeast Asia. For. Ecol. Managem. 308: 153-160.

Clarke, K.R. (1993). Non-parametric multivariate analyses of changes in community structure. Austral. J. Ecol. 18: 117-143.

Corlett, R.T. (1990). Flora and reproductive phenology of the rain forest at Bukit Timah, Singapore. J. Trop. Ecol. 6: 55-63.

Corlett, R.T. (1995). Flowering plants at Bukit Timah. Gard. Bull. Singapore (Suppl. 3): 11-27.

Davison, G.W.H. \& Chew, P.T. (2019). History of the Bukit Timah Nature Reserve, Singapore. Gard. Bull. Singapore 71 (Suppl. 1): 19-40

Davison, G.W.H., Ng, P.K.L. \& Ho, H.C. (eds) (2008). The Singapore Red Data Book: Threatened Plants and Animals of Singapore, $2^{\text {nd }}$ ed. Singapore: Nature Society (Singapore).

De Kok, R.P.J. (2015). Cryptocarya nitens (Lauraceae), a new species record for Singapore. Gard. Bull. Singapore 67: 253-259.

Gotelli, N.J. \& Colwell, R.L. (2001). Quantifying biodiversity: procedures and pitfalls in the measurements and comparisons of species richness. Ecol. Lett. 4: 379-391.

Ho, B.C., Lua, H.K., Leong, P.K.F., Lindsay, S., Seah, W.W., Bazilah Ibrahim, Loo, A.H.B., Koh, S.L., Ali Ibrahim \& Athen, P. (2018). New records and rediscoveries of vascular plants in Bukit Timah Nature Reserve, Singapore. Gard. Bull. Singapore 70: 33-55.

Keng, H. (1990). The Concise Flora of Singapore, vol. I: Gymnosperms and Dicotyledons. Singapore: Singapore University Press.

Keng, H., Chin, S.C. \& Tan, H.T.W. (1998). The Concise Flora of Singapore, vol. II: Monocotyledons. Singapore: Singapore University Press.

Khoo, M.S., Chua, S.C. \& Lum, S.K.Y. (2018). An annotated list of new records for Singapore: results from large-scale tree surveys at the Bukit Timah Nature Reserve. Gard. Bull. Singapore 70: 57-65.

King, G. (1890). Materials for a flora of the Malayan Peninsula. J. Asiat. Soc. Bengal, pt. 2, Nat. Hist. 59: 113-206.

LaFrankie, J.V., Davies, S.J., Wang, L.K., Lee, S.K. \& Lum, S.K.Y. (2005). Forest trees of Bukit Timah: Population ecology in a tropical forest fragment. Singapore: Simply Green.

LaFrankie, J.V., Lee, S.K. \& Ercelawn, A.C. (1996). Tree population structure in a tropical forest fragment in Singapore. Asian J. Trop. Biol. 2: 39-48.

Leong, P.K.F., Lee, C., Tay, F.E.L., Ang, P. \& Yam, T.W. (2017). Pinalia floribunda (Lindl.) Kuntze (Orchidaceae): Rediscovery and conservation of a species thought extinct in Singapore. Nat. Singapore 10: 67-71. 
Leong, P.K.F., Lee, C., Teo, S., Tay, F.E.L., Ang, P., Lin, E.S. \& Yam, T.W. (2018). Acriopsis ridleyi Hook.f. (Orchidaceae): Re-encounter of an orchid thought extinct since its 1889 holotype collection in Singapore. Nat. Singapore 11: 27-36.

Leong-Škorničková, J. \& Boyce, P.C. (2015). Hanguana in Singapore demystified: an overview with descriptions of three new species and a new record. Gard. Bull. Singapore 67: 1-28.

Lim, R.C.J., Lindsay, S., Middleton, D.J., Ho, B.C., Leong, P.K.F., Niissalo, M.A., van Welzen, P.C., Esser, H.-J., Ganesan, S.K., Lua, H.K., Johnson, D.M., Murray, N.A., Leong-Škorničková, J., Thomas, D.C. \& Ali Ibrahim (2018). New records and rediscoveries of plants in Singapore. Gard. Bull. Singapore 70: 67-90.

Lum, S.K.Y., Lee, S.K. \& LaFrankie, J.V. (2004). Bukit Timah Forest Dynamics Plot, Singapore. In: Losos, E.C. \& Leigh, E.G. (eds) Tropical forest diversity and dynamism: Findings from a large-scale plot network, pp. 464-473. Chicago: University of Chicago Press.

Ng, X.Y., Lim, R.C.J., Ang, W.F., Ong, K.H. \& Yeo, C.K. (2014). The conservation status in Singapore of Ampelocissus cinnamomea (Wall. ex M.A. Lawson) Planch. (Vitaceae). Nat. Singapore 7: 129-134.

Ngo, K.M., Davies, S., Hassan, N.F.N. \& Lum, S. (2016). Resilience of a forest fragment exposed to longterm isolation in Singapore. Pl. Ecol. Diversity 9: 397-407.

Niissalo, M.A. \& Leong-Škorničková, J. (2017). Hanguana podzolicola (Hanguanaceae), a new record for Singapore. Gard. Bull. Singapore 69: 157-165.

Niissalo, M.A., Wijedasa, L.S., Boyce, P.C. \& Leong-Škorničková, J. (2014). Hanguana neglecta (Hanguanaceae): a new plant species from a heavily collected and visited reserve in Singapore. Phytotaxa 188: 14-20.

Niissalo, M.A., Khew, G.S., Webb, E.L. \& Leong-Škorničková, J. (2016). Notes on Singaporean native Zingiberales II: revision of Marantaceae, with a new generic record and notes on naturalised and commonly cultivated exotic species. Phytotaxa 289: 201-224.

Niissalo, M.A., Leong-Škorničková, J., Khew, G.S. \& Webb, E.L. (2017). Very small relict populations suggest high extinction debt of gingers in primary forest fragments of a tropical city. Amer. J. Bot. 104: 182-189.

Niissalo, M.A., Leong-Škorničková, J., Webb, E.L. \& Khew, G.S. (2018). Pedigree analyses and next-generation sequencing reveal critically low regeneration in extremely threatened Zingiber singapurense (Zingiberaceae). Bot. J. Linn. Soc. 187: 346-361.

Oksanen, J., Blanchet, F.G., Friendly, M., Kindt, R., Legendre, P., McGlinn, D., Minchin, P.R., O’Hara, R.B., Simpson, G.L., Solymos, P., Stevens, M.H.H., Szoecs, E. \& Wagner, H. (2018). vegan: Community Ecology Package, R package version 2.4-6. Avalable from https://CRAN.R-project.org/package=vegan

Pasion, B.O., Roeder, M., Liu, J., Tasuda, M., Corlett, R.T., Slik, J.W.F. \& Tomlinson, K.W. (2018). Trees represent community composition of other plant life-forms, but not their diversity, abundance or responses to fragmentation. Sci. Rep. 8: 11374.

R Core Team (2016). R: A language and environment for statistical computing. Vienna: $\mathrm{R}$ Foundation for Statistical Computing. Available from https://www.R-project.org/

Ridley, H.N. (1900). The flora of Singapore. J. Straits Branch Roy. Asiat. Soc. 33: 27-196.

Ridley, H.N. (1901). Supplementary notes on the flora of Singapore. J. Straits Branch Roy. Asiat. Soc. 35: 84-90.

Rodda, M. \& Lai, J. (2018). The rediscovery of Hoya obtusifolia (Apocynaceae, Asclepiadoideae) in Singapore. Nat. Singapore 11: 45-51. 
Swan, F.R. (1988). Tree distribution patterns in the Bukit Timah Nature Reserve, Singapore. Gard. Bull. Singapore 41: 59-81.

Symington, C.F., Ashton, P.S. \& Appanah, S. (2004). Forester's manual of dipterocarps, $2^{\text {nd }}$ ed. Kuala Lumpur: Forest Research Institute Malaysia.

Tan, H.T.W., Chua, K.S. \& Turner, I.M. (1995). Rubiaceae of the Bukit Timah Nature Reserve. Gard. Bull. Singapore (Suppl. 3): 29-59.

Turner, I.M. (1996). Species loss in fragments of tropical rain forest: a review of the evidence. J. Appl. Ecology 33: 200-209.

Turner, I.M. \& Chua, K.S. (2011). Checklist of the Vascular Plant Species of the Bukit Timah Nature Reserve. Singapore: Raffles Museum of Biodiversity Research, Department of Biological Sciences, National University of Singapore.

Turner, I.M. \& Corlett, R.T. (1996). The conservation value of small, isolated fragments of lowland tropical rainforests. Trends Ecol. Evol. 11: 330-333.

Turner, I.M. \& Kumar, V.S. (2018). Flora of Singapore precursors, 4. A summary of scandent Psychotria (Rubiaceae) in Singapore and Peninsular Malaysia. Phytotaxa 361: 183-197.

Van Steenis, C.G.G.J. (1977). Bignoniaceae. In: van Steenis, C.G.G.J. (ed.) Flora Malesiana, ser. 1, vol. 8, pp. 114-186. Alphen aan den Rijn: Sijthoff \& Noordhoff.

Wee, Y.C. (1995a). Pteridophytes. Gard. Bull. Singapore (Suppl. 3): 61-69.

Wee, Y.C. (1995b). Algae. Gard. Bull. Singapore (Suppl. 3): 77-80.

Wee, Y.C. \& Haji Mohamed (1995). Bryophytes. Gard. Bull. Singapore (Suppl. 3): 71-76.

Wong, Y.K. (1987). Ecology of the trees of Bukit Timah Nature Reserve. Gard. Bull. Singapore 40: 45-76.

Yee, A.T.K., Corlett, R.T., Liew, S.C. \& Tan, H.T.W. (2011). The vegetation of Singapore - an updated map. Gard. Bull. Singapore 63: 205-212. 
Appendix I. List of species recorded for BTNR. Species are classified first into Lycophytes, Ferns, Gnetophytes, and Angiosperms and subsequently listed in alphabetical order of the families and then species.

Abbreviations: $\mathrm{A}=$ published records after Turner \& Chua (2011); $\mathrm{B}=$ published records before 2011 and omitted by Turner \& Chua (2011); C = records compiled in Turner \& Chua (2011); $\dagger=$ extinct species; $\mathrm{P}=$ Primary forest; $\mathrm{O}=$ Old Secondary forest; $\mathrm{M}=$ Maturing Secondary forest; 0,1 $=$ records in the 52 plots within the nine transects in this study; $0=$ not captured in the respective forest type; 1 = captured in the respective forest type; $\mathrm{NN}=$ non-native. Only the currently accepted names for taxa are included; synonyms are excluded. Unnamed species that are qualified with a letter (e.g. Popowia sp. A) are distinct morphospecies but for which the name is uncertain; unnamed species without a letter or number (e.g. Genianthus sp.) are where the material is simply too poor to name any further and which could be of any species.

\begin{tabular}{|c|c|c|c|c|c|c|}
\hline Family & Accepted Name & Ref. & $\mathbf{P}$ & $\mathbf{O}$ & $\mathbf{M}$ & $\mathbf{N N}$ \\
\hline \multicolumn{7}{|l|}{ LYCOPHYTES } \\
\hline Lycopodiaceae & Palhinhaea cernua (L.) Franco \& Vasc. & & 0 & 1 & 0 & \\
\hline Lycopodiaceae & Phlegmariurus phlegmaria (L.) Holub & $\mathrm{C}$ & & & & \\
\hline Selaginellaceae & Selaginella intermedia (Blume) Spring & $\mathrm{C}$ & 1 & 1 & 0 & \\
\hline Selaginellaceae & Selaginella roxburghii (Hook. \& Grev.) Spring & $\mathrm{C}$ & 1 & 1 & 0 & \\
\hline Selaginellaceae & Selaginella willdenowii (Desv. ex Poir.) Baker & $\mathrm{C}$ & 1 & 0 & 0 & \\
\hline \multicolumn{7}{|l|}{ FERNS } \\
\hline Aspleniaceae & Asplenium batuense Alderw. & $\mathrm{C}$ & & & & \\
\hline Aspleniaceae & Asplenium longissimum Blume & $\mathrm{C}$ & 0 & 1 & 1 & \\
\hline Aspleniaceae & Asplenium macrophyllum $\mathrm{Sw}$. & $\mathrm{C}$ & & & & \\
\hline Aspleniaceae & Asplenium nidus $\mathrm{L}$. & $\mathrm{C}$ & 1 & 1 & 1 & \\
\hline Aspleniaceae & Asplenium nitidum $\mathrm{Sw}$. & $\mathrm{C} \dagger$ & & & & \\
\hline Aspleniaceae & $\begin{array}{l}\text { Asplenium phyllitidis D.Don subsp. malesicum } \\
\text { Holttum }\end{array}$ & $\mathrm{C}$ & & & & \\
\hline Aspleniaceae & Asplenium tenerum G.Forst. & $\mathrm{C}$ & 1 & 1 & 0 & \\
\hline Athyriaceae & Diplazium cordifolium Blume & $\mathrm{C}$ & & & & \\
\hline Athyriaceae & Diplazium crennatoserratum (Blume) T.Moore & $\mathrm{C}$ & 1 & 1 & 0 & \\
\hline Athyriaceae & Diplazium polypodioides Blume & $\mathrm{C}$ & & & & \\
\hline Athyriaceae & Diplazium sorzogonense (C.Presl) C.Presl & $\mathrm{C}$ & & & & \\
\hline
\end{tabular}


Appendix I. Continuation.

\begin{tabular}{|c|c|c|c|c|c|c|}
\hline Family & Accepted Name & Ref. & $\mathbf{P}$ & $\mathbf{O}$ & M & $\mathbf{N N}$ \\
\hline Athyriaceae & Diplazium tomentosum Blume & $\mathrm{C}$ & 1 & 1 & 0 & \\
\hline Blechnaceae & $\begin{array}{l}\text { Blechnopsis finlaysoniana (Wall. ex Hook. \& } \\
\text { Grev.) C.Presl }\end{array}$ & $\mathrm{C}$ & 1 & 1 & 0 & \\
\hline Blechnaceae & Blechnopsis orientalis (L.) C.Presl & $\mathrm{C}$ & & & & \\
\hline Blechnaceae & Stenochlaena palustris (Burm.f.) Bedd. & $\mathrm{C}$ & 1 & 0 & 1 & \\
\hline Cyatheaceae & Alsophila glabra (Blume) Hook. & $\mathrm{C} \dagger$ & & & & \\
\hline Cyatheaceae & Alsophila latebrosa Wall. ex Hook. & $\mathrm{C}$ & 1 & 1 & 1 & \\
\hline Cyatheaceae & Sphaeropteris squamulata (Blume) R.M.Tryon & $\mathrm{C}$ & & & & \\
\hline Davalliaceae & Davallia denticulata (Burm.f.) Mett. ex Kuhn & $\mathrm{C}$ & 1 & 1 & 0 & \\
\hline Davalliaceae & Davallia heterophylla $\mathrm{Sm}$. & $\mathrm{C} \dagger$ & & & & \\
\hline Davalliaceae & Davallia repens (L.f.) Kuhn & $\mathrm{C}$ & & & & \\
\hline Davalliaceae & Davallia solida (G.Forst.) Sw. & $\mathrm{C}$ & 0 & 1 & 0 & \\
\hline Davalliaceae & Davallia triphylla Hook. & $\mathrm{C}$ & & & & \\
\hline Dennstaedtiaceae & Microlepia speluncae (L.) T.Moore & $\mathrm{C}$ & 1 & 0 & 0 & \\
\hline Dennstaedtiaceae & $\begin{array}{l}\text { Pteridium aquilinum (L.) Kuhn var. esculentum } \\
\text { (G.Forst.) Kuhn }\end{array}$ & $\mathrm{C}$ & & & & \\
\hline Dryopteridaceae & Bolbitis $\times$ singaporensis Holttum & $\mathrm{C}$ & & & & \\
\hline Dryopteridaceae & Bolbitis appendiculata (Willd.) K.Iwats. & $\mathrm{C}$ & 1 & 0 & 0 & \\
\hline Dryopteridaceae & Bolbitis sinuata (C.Presl) Hennipman & $\mathrm{C}$ & 1 & 1 & 0 & \\
\hline Dryopteridaceae & Pleocnemia irregularis (C.Presl) Holttum & $\mathrm{C}$ & 1 & 1 & 0 & \\
\hline Dryopteridaceae & $\begin{array}{l}\text { Teratophyllum aculeatum (Blume) Mett. ex } \\
\text { Kuhn }\end{array}$ & $\mathrm{C}$ & & & & \\
\hline Dryopteridaceae & Teratophyllum ludens (Fée) Holttum & $\mathrm{C}$ & 1 & 1 & 0 & \\
\hline Dryopteridaceae & $\begin{array}{l}\text { Teratophyllum rotundifoliatum (Bonap.) } \\
\text { Holttum }\end{array}$ & $\mathrm{C}$ & & & & \\
\hline Gleicheniaceae & Dicranopteris curranii Copel. & $\mathrm{C}$ & & & & \\
\hline
\end{tabular}


Appendix I. Continuation.

\begin{tabular}{|c|c|c|c|c|c|c|}
\hline Family & Accepted Name & Ref. & $\mathbf{P}$ & $\mathbf{O}$ & $\mathbf{M}$ & $\mathbf{N N}$ \\
\hline Gleicheniaceae & Dicranopteris linearis (Burm.f.) Underw. & $\mathrm{C}$ & 1 & 1 & 0 & \\
\hline Gleicheniaceae & Sticherus truncatus (Willd.) Nakai & $\mathrm{C}$ & & & & \\
\hline Hymenophyllaceae & Cephalomanes javanicum (Blume) C.Presl & $\mathrm{C}$ & 1 & 1 & 0 & \\
\hline Hymenophyllaceae & Cephalomanes obscurum (Blume) K.Iwats. & $\mathrm{C}$ & 1 & 0 & 0 & \\
\hline Hymenophyllaceae & Cephalomanes singaporianum Bosch & $\mathrm{C}$ & & & & \\
\hline Hymenophyllaceae & Crepidomanes humile (G.Forst.) Bosch & $\mathrm{C}$ & & & & \\
\hline Hymenophyllaceae & Crepidomanes minutum (Blume) K.Iwats. & $\mathrm{C} \dagger$ & & & & \\
\hline Hymenophyllaceae & Didymoglossum mindorense (Christ) K.Iwats. & $\mathrm{C}$ & 1 & 1 & 0 & \\
\hline Hymenophyllaceae & $\begin{array}{l}\text { Didymoglossum motleyi (Bosch) Ebihara \& } \\
\text { K.Iwats. }\end{array}$ & $\mathrm{C}$ & 1 & 0 & 0 & \\
\hline Hymenophyllaceae & $\begin{array}{l}\text { Didymoglossum sublimbatum (Müll.Berol.) } \\
\text { Ebihara \& K.Iwats. }\end{array}$ & $\mathrm{C}$ & & & & \\
\hline Hymenophyllaceae & Hymenophyllum denticulatum Sw. & $\mathrm{C}$ & & & & \\
\hline Lindsaeaceae & Lindsaea cultrata (Willd.) Sw. & $\mathrm{C}$ & & & & \\
\hline Lindsaeaceae & Lindsaea divergens Hook. \& Grev. & $\mathrm{C}$ & 1 & 0 & 0 & \\
\hline Lindsaeaceae & Lindsaea doryphora K.U.Kramer & $\mathrm{C}$ & & & & \\
\hline Lindsaeaceae & Lindsaea ensifolia Sw. & $\mathrm{C}$ & 1 & 1 & 0 & \\
\hline Lindsaeaceae & Lindsaea parallelogramma Alderw. & $\mathrm{C}$ & & & & \\
\hline Lindsaeaceae & Lindsaea parasitica (Roxb. ex Griff.) Hieron. & $\mathrm{C}$ & 1 & 1 & 0 & \\
\hline Lindsaeaceae & $\begin{array}{l}\text { Lindsaea repens (Bory) Thwaites var. pectinata } \\
\text { (Blume) Mett. ex Kuhn }\end{array}$ & $\mathrm{C} \dagger$ & & & & \\
\hline Lomariopsidaceae & Lomariopsis lineata (C.Presl) Holttum & $\mathrm{C}$ & 1 & 1 & 0 & \\
\hline Lygodiaceae & Lygodium flexuosum (L.) Sw. & $\mathrm{C}$ & 1 & 0 & 0 & \\
\hline Lygodiaceae & Lygodium longifolium (Willd.) Sw. & $\mathrm{C}$ & 1 & 1 & 0 & \\
\hline Lygodiaceae & Lygodium microphyllum (Cav.) R.Br. & $\mathrm{C}$ & & & & \\
\hline
\end{tabular}


Appendix I. Continuation.

\begin{tabular}{|c|c|c|c|c|c|c|}
\hline Family & Accepted Name & Ref. & $\mathbf{P}$ & $\mathbf{O}$ & $\mathbf{M}$ & NN \\
\hline Lygodiaceae & Lygodium salicifolium C.Presl & $\mathrm{C}$ & & & & \\
\hline Marattiaceae & Angiopteris evecta (G.Forst.) Hoffm. & $\mathrm{C}$ & 1 & 1 & 0 & \\
\hline Nephrolepidaceae & Nephrolepis biserrata (Sw.) Schott & $\mathrm{C}$ & 1 & 1 & 1 & \\
\hline Ophioglossaceae & Ophioglossum pendulum $\mathrm{L}$. & $\mathrm{C}$ & & & & \\
\hline Polypodiaceae & Drynaria quercifolia (L.) J.Sm. & $\mathrm{C}$ & 0 & 1 & 0 & \\
\hline Polypodiaceae & Drynaria sparsisora (Desv.) T.Moore & $\mathrm{C}$ & 1 & 1 & 0 & \\
\hline Polypodiaceae & $\begin{array}{l}\text { Goniophlebium percussum (Cav.) W.H.Wagner } \\
\& \text { Grether }\end{array}$ & $\mathrm{C}$ & 1 & 0 & 1 & \\
\hline Polypodiaceae & Lecanopteris crustacea Copel. & $\mathrm{C}$ & & & & \\
\hline Polypodiaceae & Lepisorus longifolius (Blume) Holttum & $\mathrm{C}$ & & & & \\
\hline Polypodiaceae & Microsorum membranifolium (R.Br.) Ching & $\mathrm{C}$ & & & & \\
\hline Polypodiaceae & $\begin{array}{l}\text { Platycerium coronarium (J.Koenig ex } \\
\text { O.F.Müll.) Desv. }\end{array}$ & $\mathrm{C}$ & 1 & 1 & 1 & \\
\hline Polypodiaceae & Platycerium ridleyi Christ & $\mathrm{C} \dagger$ & & & & \\
\hline Polypodiaceae & Pyrrosia angustata (Sw.) Ching & $\mathrm{C}$ & & & & \\
\hline Polypodiaceae & Pyrrosia lanceolata (L.) Farw. & $\mathrm{C}$ & & & & \\
\hline Polypodiaceae & Pyrrosia longifolia (Burm.f.) C.V.Morton & $\mathrm{C}$ & & & & \\
\hline Polypodiaceae & Pyrrosia piloselloides (L.) M.G.Price & $\mathrm{C}$ & 0 & 1 & 0 & \\
\hline Polypodiaceae & Selliguea stenophylla (Blume) Parris & $\mathrm{B} \dagger$ & & & & \\
\hline Pteridaceae & Adiantum latifolium Lam. & $\mathrm{C}$ & 0 & 1 & 1 & NN \\
\hline Pteridaceae & Antrophyum callifolium Blume & $\mathrm{C}$ & 1 & 0 & 0 & \\
\hline Pteridaceae & $\begin{array}{l}\text { Haplopteris dareicarpa (Hook.) S.Linds. \& } \\
\text { C.W.Chen }\end{array}$ & $\mathrm{C} \dagger$ & & & & \\
\hline Pteridaceae & Haplopteris elongata (Sw.) E.H.Crane & $\mathrm{C}$ & 1 & 1 & 1 & \\
\hline Pteridaceae & Haplopteris ensiformis (Sw.) E.H.Crane & $\mathrm{C}$ & 1 & 0 & 0 & \\
\hline
\end{tabular}


Appendix I. Continuation.

\begin{tabular}{|c|c|c|c|c|c|c|}
\hline Family & Accepted Name & Ref. & $\mathbf{P}$ & $\mathbf{O}$ & $\mathbf{M}$ & $\mathbf{N N}$ \\
\hline Pteridaceae & Pteris ensiformis Burm.f. & $\mathrm{C}$ & 1 & 0 & 0 & \\
\hline Pteridaceae & Pteris mertensioides Willd. & $\mathrm{C}$ & & & & \\
\hline Pteridaceae & Pteris multifida Poir. & $\mathrm{C}$ & & & & $\mathrm{NN}$ \\
\hline Pteridaceae & Pteris vittata $\mathrm{L}$. & $\mathrm{C}$ & & & & \\
\hline Pteridaceae & Syngramma alismifolia (C.Presl) J.Sm. & $\mathrm{C}$ & 1 & 1 & 0 & \\
\hline Pteridaceae & Taenitis blechnoides (Willd.) Sw. & $\mathrm{C}$ & 1 & 1 & 1 & \\
\hline Pteridaceae & Taenitis interrupta Hook. \& Grev. & $\mathrm{C}$ & & & & \\
\hline Pteridaceae & Vaginularia trichoidea Fée & $\mathrm{C} \dagger$ & & & & \\
\hline Schizaeaceae & Actinostachys digitata (L.) Wall. ex C.F.Reed & $\mathrm{C}$ & & & & \\
\hline Schizaeaceae & Schizaea dichotoma (L.) Sm. & $\mathrm{C}$ & & & & \\
\hline Tectariaceae & Tectaria angulata (Willd.) C.Chr. & $\mathrm{C}$ & & & & \\
\hline Tectariaceae & Tectaria barberi (Hook.) Copel. & $\mathrm{C}$ & 1 & 1 & 0 & \\
\hline Tectariaceae & Tectaria multicaudata (C.B.Clarke) Ching & $\mathrm{C}$ & & & & \\
\hline Tectariaceae & Tectaria nayarii Mazumdar & A & & & & \\
\hline Tectariaceae & Tectaria sagenioides (Mett.) Christenh. & $\mathrm{C}$ & 1 & 0 & 0 & \\
\hline Tectariaceae & $\begin{array}{l}\text { Tectaria singaporiana (Wall. ex Hook. \& } \\
\text { Grev.) Copel. }\end{array}$ & $\mathrm{C}$ & 1 & 1 & 0 & \\
\hline Tectariaceae & Tectaria semipinnata (Roxb.) C.V.Morton & $\mathrm{C}$ & 1 & 1 & 0 & \\
\hline Thelypteridaceae & Amblovenatum opulentum (Kaulf.) J.P.Roux & $\mathrm{C}$ & 1 & 0 & 0 & \\
\hline Thelypteridaceae & Christella arida (D.Don) Holttum & $\mathrm{C}$ & & & & \\
\hline Thelypteridaceae & Christella parasitica (L.) H.Lév. & $\mathrm{C}$ & & & & \\
\hline Thelypteridaceae & Christella subpubescens (Blume) Holttum & $\mathrm{C}$ & 0 & 0 & 1 & \\
\hline Thelypteridaceae & $\begin{array}{l}\text { Mesophlebion chlamydophorum (Rosenst. ex } \\
\text { C.Chr.) Holttum }\end{array}$ & $\mathrm{C}$ & & & & \\
\hline
\end{tabular}


Appendix I. Continuation.

\begin{tabular}{|c|c|c|c|c|c|c|}
\hline Family & Accepted Name & Ref. & $\mathbf{P}$ & $\mathbf{O}$ & M & $\mathbf{N N}$ \\
\hline Thelypteridaceae & Mesophlebion motleyanum (Hook.) Holttum & $\mathrm{C}$ & 1 & 1 & 0 & \\
\hline Thelypteridaceae & Pneumatopteris truncata (Poir.) Holttum & $\mathrm{C}$ & & & & \\
\hline Thelypteridaceae & Pronephrium menisciicarpon (Blume) Holttum & $\mathrm{C}$ & & & & \\
\hline Thelypteridaceae & Pronephrium repandum (Fée) Holttum & $\mathrm{C}$ & 1 & 1 & 0 & \\
\hline Thelypteridaceae & Pronephrium triphyllum (Sw.) Holttum & $\mathrm{C}$ & 0 & 1 & 1 & \\
\hline Thelypteridaceae & $\begin{array}{l}\text { Sphaerostephanos heterocarpus (Blume) } \\
\text { Holttum }\end{array}$ & $\mathrm{C}$ & 1 & 0 & 0 & \\
\hline Thelypteridaceae & Sphaerostephanos polycarpus (Blume) Copel. & $\mathrm{C}$ & 1 & 0 & 0 & \\
\hline Thelypteridaceae & Sphaerostephanos unitus (L.) Holttum & $\mathrm{C}$ & & & & \\
\hline \multicolumn{7}{|l|}{ GNETOPHYTES } \\
\hline Gnetaceae & Gnetum macrostachyum Hook.f. & $\mathrm{C}$ & 1 & 0 & 0 & \\
\hline Gnetaceae & Gnetum microcarpum Blume & $\mathrm{C}$ & 1 & 1 & 0 & \\
\hline \multicolumn{7}{|l|}{ ANGIOSPERMS } \\
\hline Acanthaceae & $\begin{array}{l}\text { Asystasia gangetica }(\mathrm{L} .) \text { T.Anderson subsp. } \\
\text { micrantha (Nees) Ensermu }\end{array}$ & & 0 & 0 & 1 & $\mathrm{NN}$ \\
\hline Acanthaceae & Staurogyne griffithiana Kuntze & $\mathrm{C} \dagger$ & & & & \\
\hline Acanthaceae & Staurogyne setigera (Nees) Kuntze & $\mathrm{C}$ & 0 & 1 & 0 & \\
\hline Acanthaceae & $\begin{array}{l}\text { Strobilanthes reptans (G.Forst.) Moylan ex } \\
\text { Y.F.Deng \& J.R.I.Wood }\end{array}$ & & 1 & 0 & 1 & $\mathrm{NN}$ \\
\hline Acanthaceae & Thunbergia fragrans Roxb. & & 0 & 0 & 1 & $\mathrm{NN}$ \\
\hline Acanthaceae & (unidentified) & & 0 & 1 & 0 & \\
\hline Achariaceae & Ryparosa hullettii King & $\mathrm{C}$ & 1 & 1 & 0 & \\
\hline Achariaceae & Ryparosa scortechinii King & B & 1 & 0 & 0 & \\
\hline Actinidiaceae & Saurauia pentapetala (Jack) Hoogland & $\mathrm{C} \dagger$ & & & & \\
\hline Amaranthaceae & Cyathula prostrata (L.) Blume & B & 0 & 0 & 1 & \\
\hline
\end{tabular}


Appendix I. Continuation.

\begin{tabular}{|c|c|c|c|c|c|c|}
\hline Family & Accepted Name & Ref. & $\mathbf{P}$ & $\mathbf{O}$ & $\mathbf{M}$ & $\mathbf{N N}$ \\
\hline Anacardiaceae & Bouea oppositifolia (Roxb.) Meisn. & $\mathrm{C}$ & 1 & 1 & 0 & \\
\hline Anacardiaceae & Buchanania arborescens (Blume) Blume & & 1 & 0 & 0 & \\
\hline Anacardiaceae & Buchanania sessifolia Blume & $\mathrm{C}$ & 1 & 1 & 0 & \\
\hline Anacardiaceae & Campnosperma auriculatum (Blume) Hook.f. & $\mathrm{C}$ & 1 & 1 & 1 & \\
\hline Anacardiaceae & Campnosperma squamatum Ridl. & $\mathrm{C}$ & 1 & 1 & 0 & \\
\hline Anacardiaceae & Dracontomelon dao (Blanco) Merr. \& Rolfe & & 1 & 1 & 0 & \\
\hline Anacardiaceae & Gluta malayana (Corner) Ding Hou & A & & & & \\
\hline Anacardiaceae & Gluta wallichii (Hook.f.) Ding Hou & $\mathrm{C}$ & 1 & 1 & 0 & \\
\hline Anacardiaceae & Mangifera foetida Lour. & $\mathrm{C}$ & & & & \\
\hline Anacardiaceae & Mangifera gracilipes Hook.f. & A & & & & \\
\hline Anacardiaceae & Mangifera griffithii Hook.f. & $\mathrm{C}$ & & & & \\
\hline Anacardiaceae & Mangifera indica $\mathrm{L}$. & & 1 & 0 & 0 & $\mathrm{NN}$ \\
\hline Anacardiaceae & $\begin{array}{l}\text { Mangifera odorata } \text { Griff. [possibly a hybrid } M \text {. } \\
\text { foetida } \times M \text {. } \text { indica }]\end{array}$ & $\mathrm{B}$ & 0 & 0 & 1 & $\mathrm{NN}$ \\
\hline Anacardiaceae & Mangifera subsessilifolia Kosterm. & $\mathrm{C}$ & & & & \\
\hline Anacardiaceae & Melanochyla caesia (Blume) Ding Hou & & 1 & 0 & 0 & \\
\hline Anacardiaceae & Parishia insignis Hook.f. & $\mathrm{C}$ & 1 & 0 & 0 & \\
\hline Anacardiaceae & Parishia maingayi Hook.f. & $\mathrm{C}$ & 1 & 1 & 0 & \\
\hline Anacardiaceae & Parishia paucijuga Engl. & $\mathrm{C}$ & 1 & 1 & 0 & \\
\hline Anacardiaceae & $\begin{array}{l}\text { Swintonia schwenkii (Teijsm. \& Binn.) Teijsm. } \\
\& \text { Binn. }\end{array}$ & $\mathrm{C}$ & 1 & 1 & 0 & \\
\hline Anisophylleaceae & Anisophyllea disticha (Jack) Baill. & $\mathrm{C}$ & 1 & 1 & 1 & \\
\hline Annonaceae & Alphonsea johorensis J.Sinclair & A & 0 & 1 & 0 & \\
\hline Annonaceae & Alphonsea maingayi Hook.f. \& Thomson & & 1 & 1 & 0 & \\
\hline
\end{tabular}


Appendix I. Continuation.

\begin{tabular}{|c|c|c|c|c|c|c|}
\hline Family & Accepted Name & Ref. & $\mathbf{P}$ & $\mathbf{O}$ & $\mathbf{M}$ & $\mathbf{N N}$ \\
\hline Annonaceae & Artabotrys costatus King & & 1 & 1 & 0 & \\
\hline Annonaceae & Artabotrys crassifolius Hook.f. \& Thomson & $\mathrm{C}$ & 1 & 1 & 0 & \\
\hline Annonaceae & Artabotrys maingayi Hook.f. \& Thomson & & 0 & 1 & 0 & \\
\hline Annonaceae & Artabotrys scortechinii King & $\mathrm{A}$ & & & & \\
\hline Annonaceae & Artabotrys suaveolens (Blume) Blume & $\mathrm{C}$ & 1 & 1 & 1 & \\
\hline Annonaceae & Artabotrys wrayi King & $\mathrm{C}$ & 0 & 1 & 0 & \\
\hline Annonaceae & $\begin{array}{l}\text { Dasymaschalon dasymaschalum (Blume) } \\
\text { I.M.Turner }\end{array}$ & $\mathrm{C}$ & 1 & 1 & 0 & \\
\hline Annonaceae & $\begin{array}{l}\text { Dendrokingstonia nervosa (Hook.f. \& } \\
\text { Thomson) Rauschert }\end{array}$ & A & & & & \\
\hline Annonaceae & Desmos chinensis Lour. & & 1 & 1 & 0 & \\
\hline Annonaceae & Desmos dumosus (Roxb.) Saff. & & 1 & 1 & 0 & \\
\hline Annonaceae & $\begin{array}{l}\text { Drepananthus ramuliflorus Maingay ex Hook.f. } \\
\& \text { Thomson }\end{array}$ & $\mathrm{C}$ & 1 & 1 & 0 & \\
\hline Annonaceae & $\begin{array}{l}\text { Drepananthus ridleyi (King) Survesw. \& } \\
\text { R.M.K.Saunders }\end{array}$ & $\mathrm{C}$ & 0 & 1 & 0 & \\
\hline Annonaceae & Fissistigma fulgens (Hook.f. \& Thomson) Merr. & $\mathrm{C}$ & 1 & 1 & 0 & \\
\hline Annonaceae & $\begin{array}{l}\text { Fissistigma lanuginosum (Hook.f. \& Thomson) } \\
\text { Merr. }\end{array}$ & $\mathrm{C}$ & 1 & 1 & 0 & \\
\hline Annonaceae & Fissistigma ovoideum (King) Merr. & $\mathrm{C}$ & & & & \\
\hline Annonaceae & Friesodielsia biglandulosa (Blume) Steenis & & 0 & 1 & 0 & \\
\hline Annonaceae & Friesodielsia borneensis (Miq.) Steenis & $\mathrm{C}$ & 1 & 0 & 1 & \\
\hline Annonaceae & $\begin{array}{l}\text { Friesodielsia latifolia (Hook.f. \& Thomson) } \\
\text { Steenis }\end{array}$ & & 0 & 1 & 1 & \\
\hline Annonaceae & $\begin{array}{l}\text { Goniothalamus macrophyllus (Blume) Hook.f. } \\
\& \text { Thomson }\end{array}$ & $\mathrm{C}$ & 1 & 1 & 0 & \\
\hline Annonaceae & Goniothalamus malayanus Hook.f. \& Thomson & $\mathrm{C}$ & & & & \\
\hline
\end{tabular}


Appendix I. Continuation.

\begin{tabular}{|c|c|c|c|c|c|c|}
\hline Family & Accepted Name & Ref. & $\mathbf{P}$ & $\mathbf{O}$ & $\mathbf{M}$ & $\mathbf{N N}$ \\
\hline Annonaceae & Goniothalamus ridleyi King & $\mathrm{C}$ & & & & \\
\hline Annonaceae & Goniothalamus tapis Miq. & $\mathrm{C}$ & 0 & 1 & 0 & \\
\hline Annonaceae & $\begin{array}{l}\text { Huberantha jenkinsii (Hook.f. \& Thomson) } \\
\text { Chaowasku }\end{array}$ & $\mathrm{C}$ & 1 & 0 & 0 & \\
\hline Annonaceae & $\begin{array}{l}\text { Huberantha rumphii (Blume ex Hensch.) } \\
\text { Chaowasku }\end{array}$ & $\mathrm{C}$ & & & & \\
\hline Annonaceae & $\begin{array}{l}\text { Maasia glauca (Hassk.) Mols, Kessler \& } \\
\text { Rogstad }\end{array}$ & $\mathrm{C}$ & 0 & 1 & 0 & \\
\hline Annonaceae & $\begin{array}{l}\text { Maasia hypoleuca (Hook.f. \& Thomson) Mols, } \\
\text { Kessler \& Rogstad }\end{array}$ & $\mathrm{C}$ & 1 & 1 & 0 & \\
\hline Annonaceae & $\begin{array}{l}\text { Maasia sumatrana (Miq.) Mols, Kessler \& } \\
\text { Rogstad }\end{array}$ & & 1 & 0 & 0 & \\
\hline Annonaceae & Meiogyne virgata (Blume) Miq. & $\mathrm{C}$ & 1 & 1 & 0 & \\
\hline Annonaceae & Mezzettia parviflora Becc. & $\mathrm{C}$ & 1 & 1 & 0 & \\
\hline Annonaceae & Miliusa eupoda (Miq.) I.M.Turner & $\mathrm{C} \dagger$ & & & & \\
\hline Annonaceae & Mitrella kentii (Blume) Miq. & $\mathrm{C}$ & 1 & 1 & 0 & \\
\hline Annonaceae & $\begin{array}{l}\text { Monoon anomalum (Becc.) B.Xue \& } \\
\text { R.M.K.Saunders }\end{array}$ & $\mathrm{C}$ & 1 & 0 & 0 & \\
\hline Annonaceae & $\begin{array}{l}\text { Monoon borneense (H.Okada) B.Xue \& } \\
\text { R.M.K.Saunders }\end{array}$ & $\mathrm{C}$ & 1 & 1 & 0 & \\
\hline Annonaceae & $\begin{array}{l}\text { Monoon hookerianum (King) B.Xue \& } \\
\text { R.M.K.Saunders }\end{array}$ & $\mathrm{C}$ & & & & \\
\hline Annonaceae & Monoon lateriflorum (Blume) Miq. & $\mathrm{C}$ & 1 & 0 & 0 & \\
\hline Annonaceae & $\begin{array}{l}\text { Phaeanthus intermedius (P.Parm.) I.M.Turner } \\
\text { \& Veldkamp }\end{array}$ & $\mathrm{C}$ & 1 & 1 & 0 & \\
\hline Annonaceae & Polyalthia angustissima Ridl. & $\mathrm{C}$ & 1 & 1 & 0 & \\
\hline Annonaceae & Polyalthia cauliflora Hook.f. \& Thomson & $\mathrm{C}$ & 1 & 1 & 1 & \\
\hline Annonaceae & Popowia fusca King & $\mathrm{C}$ & 1 & 1 & 0 & \\
\hline
\end{tabular}


Appendix I. Continuation.

\begin{tabular}{|c|c|c|c|c|c|c|}
\hline Family & Accepted Name & Ref. & $\mathbf{P}$ & $\mathbf{O}$ & $\mathbf{M}$ & $\mathbf{N N}$ \\
\hline Annonaceae & Popowia pisocarpa (Blume) Endl. & $\mathrm{C}$ & 1 & 0 & 0 & \\
\hline Annonaceae & $\begin{array}{l}\text { Popowia tomentosa Maingay ex Hook.f. \& } \\
\text { Thomson }\end{array}$ & $\mathrm{C}$ & 0 & 1 & 0 & \\
\hline Annonaceae & Popowia sp. A & & 0 & 1 & 0 & \\
\hline Annonaceae & $\begin{array}{l}\text { Pyramidanthe prismatica (Hook.f. \& Thomson) } \\
\text { Merr. }\end{array}$ & $\mathrm{C}$ & 0 & 0 & 1 & \\
\hline Annonaceae & $\begin{array}{l}\text { Uvaria cuneifolia (Hook.f. \& Thomson) } \\
\text { L.L.Zhou, Y.C.F.Su \& R.M.K.Saunders }\end{array}$ & & 1 & 0 & 0 & \\
\hline Annonaceae & Uvaria curtisii King & $\mathrm{C}$ & 1 & 1 & 0 & \\
\hline Annonaceae & Uvaria grandiflora Roxb. ex Hornem. & $\mathrm{C}$ & & & & \\
\hline Annonaceae & $\begin{array}{l}\text { Uvaria griffithii L.L.Zhou, Y.C.F.Su \& } \\
\text { R.M.K.Saunders }\end{array}$ & $\mathrm{C}$ & 1 & 1 & 0 & \\
\hline Annonaceae & Uvaria hirsuta Jack & & 1 & 1 & 0 & \\
\hline Annonaceae & Uvaria leptopoda (King) R.E.Fr. & $\mathrm{C}$ & & & & \\
\hline Annonaceae & Uvaria littoralis (Blume) Blume & $\mathrm{C}$ & & & & \\
\hline Annonaceae & Uvaria lobbiana Hook.f. \& Thomson & & 0 & 1 & 0 & \\
\hline Annonaceae & Uvaria pauciovulata Hook.f. \& Thomson & $\mathrm{C}$ & 1 & 0 & 0 & \\
\hline Annonaceae & Xylopia caudata Hook.f. \& Thomson & $\mathrm{C}$ & 1 & 0 & 0 & \\
\hline Annonaceae & Xylopia ferruginea (Hook.f. \& Thomson) Baill. & $\mathrm{C}$ & 0 & 1 & 0 & \\
\hline Annonaceae & $\begin{array}{l}\text { Xylopia magna Maingay ex Hook.f. \& } \\
\text { Thomson }\end{array}$ & $\mathrm{C}$ & & & & \\
\hline Annonaceae & Xylopia malayana Hook.f. \& Thomson & $\mathrm{C}$ & 1 & 1 & 0 & \\
\hline Apocynaceae & Alstonia angustifolia Wall. ex A.DC. & $\mathrm{C}$ & 1 & 1 & 0 & \\
\hline Apocynaceae & Alstonia angustiloba Miq. & $\mathrm{C}$ & 1 & 1 & 1 & \\
\hline Apocynaceae & Alyxia reinwardtii Blume & $\mathrm{C}$ & & & & \\
\hline Apocynaceae & Anodendron candolleanum Wight & & 1 & 1 & 0 & \\
\hline
\end{tabular}


Appendix I. Continuation.

\begin{tabular}{|c|c|c|c|c|c|c|}
\hline Family & Accepted Name & Ref. & $\mathbf{P}$ & $\mathbf{O}$ & $\mathbf{M}$ & $\mathbf{N N}$ \\
\hline Apocynaceae & Cynanchum ovalifolium Wight & $\mathrm{C}$ & & & & \\
\hline Apocynaceae & Dischidia cochleata Blume & $\mathrm{C}$ & & & & \\
\hline Apocynaceae & Dyera costulata (Miq.) Hook.f. & $\mathrm{C}$ & 1 & 1 & 0 & \\
\hline Apocynaceae & Epigynum sp. A & & 1 & 1 & 0 & \\
\hline Apocynaceae & Genianthus maingayi Hook.f. & $\mathrm{B} \dagger$ & & & & \\
\hline Apocynaceae & Genianthus sp. & & 1 & 1 & 0 & \\
\hline Apocynaceae & Hoya latifolia G.Don & & 1 & 0 & 0 & \\
\hline Apocynaceae & Hoya obtusifolia Wight & B & & & & \\
\hline Apocynaceae & Hoya verticillata (Vahl) G.Don var. verticillata & $\mathrm{C}$ & & & & \\
\hline Apocynaceae & Leuconotis griffithii Hook.f. & $\mathrm{C}$ & 1 & 1 & 0 & \\
\hline Apocynaceae & Micrechites serpyllifolius (Blume) Kosterm. & $\mathrm{C}$ & 1 & 1 & 0 & \\
\hline Apocynaceae & Parsonsia sp. A & & 1 & 0 & 0 & \\
\hline Apocynaceae & Strophanthus caudatus (L.) Kurz & & 1 & 1 & 0 & \\
\hline Apocynaceae & Tabernaemontana corymbosa Roxb. ex Wall. & & 0 & 1 & 0 & \\
\hline Apocynaceae & Urceola brachysepala Hook.f. & $\mathrm{C}$ & 1 & 1 & 0 & \\
\hline Apocynaceae & Urceola elastica Roxb. & $\mathrm{C}$ & 1 & 0 & 0 & \\
\hline Apocynaceae & $\begin{array}{l}\text { Urceola polyneura (Hook.f.) D.J.Middleton \& } \\
\text { Livsh. }\end{array}$ & & 1 & 1 & 0 & \\
\hline Apocynaceae & Urceola torulosa Hook.f. & $\mathrm{C}$ & & & & \\
\hline Apocynaceae & Urceola sp. & & 1 & 0 & 0 & \\
\hline Apocynaceae & Willughbeia coriacea Wall. & $\mathrm{B}$ & 1 & 1 & 0 & \\
\hline Apocynaceae & Willughbeia edulis Roxb. & $\mathrm{C}+$ & & & & \\
\hline Apocynaceae & Willughbeia flavescens Dyer ex Hook.f. & $\mathrm{C}$ & 0 & 1 & 0 & \\
\hline Apocynaceae & Willughbeia tenuiflora Dyer ex Hook.f. & $\mathrm{C}$ & & & & \\
\hline
\end{tabular}


Appendix I. Continuation.

\begin{tabular}{|c|c|c|c|c|c|c|}
\hline Family & Accepted Name & Ref. & $\mathbf{P}$ & $\mathbf{O}$ & $\mathbf{M}$ & $\mathbf{N N}$ \\
\hline Apocynaceae & Wrightia laevis Hook.f. & $\mathrm{C}$ & 1 & 1 & 0 & \\
\hline Aquifoliaceae & Ilex cymosa Blume & & 1 & 0 & 0 & \\
\hline Aquifoliaceae & Ilex latifolia Thunb. & $\mathrm{C}$ & 0 & 1 & 0 & \\
\hline Aquifoliaceae & Ilex maingayi Hook.f. & $\mathrm{C}$ & & & & \\
\hline Araceae & Aglaonema nebulosum N.E.Br. & $\mathrm{C}$ & & & & \\
\hline Araceae & Aglaonema nitidum (Jack) Kunth & $\mathrm{B}$ & 0 & 1 & 0 & \\
\hline Araceae & Aglaonema simplex Blume & $\mathrm{C}$ & 0 & 1 & 0 & \\
\hline Araceae & Alocasia longiloba Miq. & $\mathrm{C}$ & 1 & 1 & 0 & \\
\hline Araceae & Amorphophallus prainii Hook.f. & $\mathrm{C}$ & & & & \\
\hline Araceae & Amydrium medium (Zoll. \& Moritzi) Nicolson & $\mathrm{C}$ & 1 & 1 & 0 & \\
\hline Araceae & $\begin{array}{l}\text { Anadendrum microstachyum (de Vriese \& } \\
\text { Miq.) Backer \& Alderw. }\end{array}$ & $\mathrm{C}$ & 1 & 1 & 0 & \\
\hline Araceae & Cryptocoryne griffithii Schott & B & & & & \\
\hline Araceae & Cryptocoryne $\times$ timahensis Bastm. & $\mathrm{C}$ & & & & \\
\hline Araceae & $\begin{array}{l}\text { Dieffenbachia seguine (Jacq.) Schott var. } \\
\text { seguine }\end{array}$ & & 0 & 0 & 1 & NN \\
\hline Araceae & Dieffenbachia sp. & & 0 & 0 & 1 & $\mathrm{NN}$ \\
\hline Araceae & $\begin{array}{l}\text { Epipremnum aureum (Linden ex André) } \\
\text { G.S.Bunting }\end{array}$ & & 0 & 0 & 1 & NN \\
\hline Araceae & Epipremnum giganteum (Roxb.) Schott & $\mathrm{C}$ & 1 & 1 & 1 & \\
\hline Araceae & Epipremnum pinnatum (L.) Engl. & B & 1 & 0 & 1 & \\
\hline Araceae & Homalomena griffithii (Schott) Hook.f. & $\mathrm{C}$ & 1 & 0 & 0 & \\
\hline Araceae & Homalomena humilis (Jack) Hook.f. & $\mathrm{C}$ & & & & \\
\hline Araceae & Homalomena pendula (Blume) Bakh.f. & $\mathrm{C}$ & & & & \\
\hline Araceae & Homalomena sagittifolia Jungh. ex Schott & $\mathrm{C}$ & & & & \\
\hline
\end{tabular}


Appendix I. Continuation.

\begin{tabular}{|c|c|c|c|c|c|c|}
\hline Family & Accepted Name & Ref. & $\mathbf{P}$ & $\mathbf{O}$ & $\mathbf{M}$ & $\mathbf{N N}$ \\
\hline Araceae & Lasia spinosa (L.) Thwaites & & 0 & 0 & 1 & \\
\hline Araceae & Rhaphidophora korthalsii Schott & $\mathrm{C}$ & 1 & 1 & 0 & \\
\hline Araceae & Rhaphidophora lobbii Schott & $\mathrm{C}$ & 1 & 0 & 0 & \\
\hline Araceae & Rhaphidophora maingayi Hook.f. & $\mathrm{B}$ & 1 & 1 & 0 & \\
\hline Araceae & Rhaphidophora minor Hook.f. & & 1 & 0 & 0 & \\
\hline Araceae & Rhaphidophora montana (Blume) Schott & $\mathrm{C}$ & 1 & 1 & 0 & \\
\hline Araceae & Rhaphidophora sylvestris (Blume) Engl. & $\mathrm{C}$ & & & & \\
\hline Araceae & $\begin{array}{l}\text { Schismatoglottis calyptrata (Roxb.) Zoll. \& } \\
\text { Moritzi }\end{array}$ & $\mathrm{C}$ & 1 & 1 & 0 & \\
\hline Araceae & Schismatoglottis wallichii (Roxb.) Hook.f. & $\mathrm{C}$ & 1 & 1 & 0 & \\
\hline Araceae & Schismatoglottis sp. A & & 1 & 1 & 0 & \\
\hline Araceae & Scindapsus hederaceus Miq. & $\mathrm{B}$ & 1 & 1 & 0 & \\
\hline Araceae & Scindapsus lucens Bogner \& P.C.Boyce & A & 1 & 0 & 0 & \\
\hline Araceae & Scindapsus pictus Hassk. & B & 1 & 1 & 0 & \\
\hline Araceae & Syngonium podophyllum Schott & & 0 & 0 & 1 & $\mathrm{NN}$ \\
\hline Araliaceae & $\begin{array}{l}\text { Polyscias diversifolia (Blume) Lowry \& } \\
\text { G.M.Plunkett }\end{array}$ & $\mathrm{C}$ & 0 & 1 & 1 & \\
\hline Araliaceae & Schefflera elliptica (Blume) Harms & $\mathrm{C}$ & & & & \\
\hline Araliaceae & Schefflera lanceolata Ridl. & $\mathrm{C} \dagger$ & & & & \\
\hline Arecaceae & Calamus insignis Griff. & $\mathrm{C}$ & 1 & 1 & 1 & \\
\hline Arecaceae & Calamus javensis Blume & $\mathrm{C}$ & 1 & 1 & 0 & \\
\hline Arecaceae & Calamus lobbianus Becc. & $\mathrm{C}$ & & & & \\
\hline Arecaceae & Calamus ornatus Blume & $\mathrm{C}$ & 0 & 1 & 0 & \\
\hline Arecaceae & Calamus oxleyanus Teijsm. \& Binn. ex Miq. & $\mathrm{C}$ & 1 & 1 & 1 & \\
\hline Arecaceae & Calamus paspalanthus Becc. & $\mathrm{C}$ & 1 & 0 & 0 & \\
\hline
\end{tabular}


Appendix I. Continuation.

\begin{tabular}{|c|c|c|c|c|c|c|}
\hline Family & Accepted Name & Ref. & $\mathbf{P}$ & $\mathbf{O}$ & $\mathbf{M}$ & NN \\
\hline Arecaceae & Calamus plicatus Blume & $\mathrm{C}$ & 1 & 1 & 0 & \\
\hline Arecaceae & Calamus ridleyanus Becc. & & 1 & 1 & 0 & \\
\hline Arecaceae & Caryota mitis Lour. & $\mathrm{C}$ & 1 & 1 & 1 & \\
\hline Arecaceae & Daemonorops angustifolia (Griff.) Mart. & B & 1 & 1 & 0 & \\
\hline Arecaceae & Daemonorops didymophylla Becc. & $\mathrm{C}$ & 1 & 1 & 0 & \\
\hline Arecaceae & Daemonorops geniculata (Griff.) Mart. & B & & & & \\
\hline Arecaceae & Daemonorops grandis (Griff.) Mart. & $\mathrm{C}$ & 1 & 1 & 0 & \\
\hline Arecaceae & Daemonorops hirsuta Blume & $\mathrm{C}$ & 1 & 0 & 0 & \\
\hline Arecaceae & Daemonorops lewisiana (Griff.) Mart. & $\mathrm{C} \dagger$ & & & & \\
\hline Arecaceae & Daemonorops longipes (Griff.) Mart. & $\mathrm{C}$ & 1 & 0 & 0 & \\
\hline Arecaceae & Daemonorops micracantha (Griff.) Becc. & $\mathrm{C}$ & & & & \\
\hline Arecaceae & Daemonorops periacantha Miq. & $\mathrm{C}$ & 1 & 1 & 0 & \\
\hline Arecaceae & Elaeis guineensis Jacq. & & 1 & 0 & 1 & $\mathrm{NN}$ \\
\hline Arecaceae & Eleiodoxa conferta (Griff.) Burret & & 0 & 1 & 0 & \\
\hline Arecaceae & Iguanura geonomiformis Mart. & $\mathrm{C}$ & & & & \\
\hline Arecaceae & Korthalsia echinometra Becc. & $\mathrm{C}$ & 1 & 0 & 0 & \\
\hline Arecaceae & Korthalsia flagellaris Miq. & & 1 & 0 & 0 & \\
\hline Arecaceae & Korthalsia rigida Blume & $\mathrm{C}$ & 1 & 1 & 0 & \\
\hline Arecaceae & Korthalsia rostrata Blume & $\mathrm{C}$ & 1 & 1 & 0 & \\
\hline Arecaceae & Licuala ferruginea Becc. & $\mathrm{C}$ & 1 & 0 & 0 & \\
\hline Arecaceae & Myrialepis paradoxa (Kurz) J.Dransf. & $\mathrm{C}$ & 1 & 0 & 0 & \\
\hline Arecaceae & $\begin{array}{l}\text { Nenga pumila (Blume) H.Wendl. var. } \\
\text { pachystachya (Blume) Fernando }\end{array}$ & & 0 & 1 & 0 & \\
\hline Arecaceae & Oncosperma horridum (Griff.) Scheff. & $\mathrm{C}$ & 1 & 1 & 1 & \\
\hline
\end{tabular}


Appendix I. Continuation.

\begin{tabular}{|c|c|c|c|c|c|c|}
\hline Family & Accepted Name & Ref. & $\mathbf{P}$ & $\mathbf{O}$ & $\mathbf{M}$ & $\mathbf{N N}$ \\
\hline Arecaceae & Orania sylvicola (Griff.) H.E.Moore & $\mathrm{C}$ & & & & \\
\hline Arecaceae & $\begin{array}{l}\text { Pinanga auriculata Becc. var. leucocarpa } \\
\text { C.K.Lim }\end{array}$ & $\mathrm{C}$ & & & & \\
\hline Arecaceae & Pinanga limosa Ridl. & $\mathrm{C}^{\dagger}$ & & & & \\
\hline Arecaceae & Pinanga malaiana (Mart.) Scheff. & $\mathrm{C}$ & & & & \\
\hline Arecaceae & Pinanga pectinata Becc. & $\mathrm{B} \dagger$ & & & & \\
\hline Arecaceae & Plectocomia elongata Mart. ex Blume & & 1 & 1 & 1 & \\
\hline Arecaceae & Plectocomiopsis geminiflora (Griff.) Becc. & & 0 & 1 & 0 & \\
\hline Arecaceae & $\begin{array}{l}\text { Ptychosperma macarthurii (H.Wendl. ex } \\
\text { H.J.Veitch) H.Wendl. ex Hook.f. }\end{array}$ & & 0 & 0 & 1 & $\mathrm{NN}$ \\
\hline Arecaceae & Rhopaloblaste singaporensis (Becc.) Hook.f. & $\mathrm{C}$ & 1 & 1 & 0 & \\
\hline Arecaceae & Salacca affinis Griff. & $\mathrm{C} \dagger$ & & & & \\
\hline Aristolochiaceae & Thottea grandiflora Rottb. & $\mathrm{C}$ & & & & \\
\hline Asparagaceae & Cordyline fruticosa (L.) A.Chev. & & 0 & 0 & 1 & NN \\
\hline Asparagaceae & Dracaena cantleyi Baker & $\mathrm{C}$ & 0 & 1 & 0 & \\
\hline Asparagaceae & Dracaena elliptica Thunb. & & 1 & 0 & 0 & \\
\hline Asparagaceae & Dracaena fragrans (L.) Ker Gawl. & & 0 & 1 & 1 & $\mathrm{NN}$ \\
\hline Asparagaceae & Dracaena granulata Hook.f. & $\mathrm{C}$ & 0 & 1 & 0 & \\
\hline Asparagaceae & Dracaena maingayi Hook.f. & $\mathrm{C}$ & 1 & 1 & 0 & \\
\hline Asparagaceae & Dracaena porteri Baker & & 1 & 0 & 0 & \\
\hline Asparagaceae & Dracaena sanderiana Mast. & & 0 & 0 & 1 & $\mathrm{NN}$ \\
\hline Asparagaceae & Dracaena singapurensis Ridl. & $\mathrm{C}^{\dagger}$ & & & & \\
\hline Asparagaceae & Dracaena sp. & & 1 & 0 & 0 & \\
\hline Asteraceae & Blumea riparia (Blume) DC. & B & & & & \\
\hline Asteraceae & Gynura procumbens (Lour.) Merr. & B & & & & \\
\hline
\end{tabular}


Appendix I. Continuation.

\begin{tabular}{|c|c|c|c|c|c|c|}
\hline Family & Accepted Name & Ref. & $\mathbf{P}$ & $\mathbf{O}$ & $\mathbf{M}$ & $\mathbf{N N}$ \\
\hline Asteraceae & Struchium sparganophorum (L.) Kuntze & & 0 & 0 & 1 & $\mathrm{NN}$ \\
\hline Asteraceae & Vernonia arborea Buch.-Ham. & $\mathrm{C}$ & 0 & 1 & 0 & \\
\hline Bignoniaceae & Deplanchea bancana (Scheff.) Steenis & $\mathrm{C} \dagger$ & & & & \\
\hline Bignoniaceae & Fernandoa adenophylla (G.Don) Steenis & $\mathrm{B}$ & & & & \\
\hline Bignoniaceae & $\begin{array}{l}\text { Radermachera quadripinnata (Blanco) Seem. } \\
\text { subsp. lobbii (Teijsm. \& Binn.) I.M.Turner }\end{array}$ & $\mathrm{C}$ & 1 & 0 & 0 & \\
\hline Bignoniaceae & Spathodea campanulata P.Beauv. & & 0 & 1 & 1 & $\mathrm{NN}$ \\
\hline Bignoniaceae & Stereospermum tetragonum DC. & $\mathrm{C}$ & & & & \\
\hline Burmanniaceae & Burmannia championii Thwaites & $\mathrm{C}$ & & & & \\
\hline Burmanniaceae & Gymnosiphon aphyllus Blume & $\mathrm{C} \dagger$ & & & & \\
\hline Burseraceae & Canarium grandifolium (Ridl.) H.J.Lam & $\mathrm{C}$ & & & & \\
\hline Burseraceae & Canarium littorale Blume & $\mathrm{C}$ & 1 & 1 & 0 & \\
\hline Burseraceae & Canarium patentinervium Miq. & $\mathrm{C}$ & 1 & 1 & 0 & \\
\hline Burseraceae & Canarium pilosum A.W.Benn. & $\mathrm{C}$ & 1 & 1 & 0 & \\
\hline Burseraceae & Dacryodes costata (A.W.Benn.) H.J.Lam & $\mathrm{C}$ & 1 & 0 & 0 & \\
\hline Burseraceae & Dacryodes laxa (A.W.Benn.) H.J.Lam & $\mathrm{C}$ & 1 & 0 & 0 & \\
\hline Burseraceae & Dacryodes longifolia (King) H.J.Lam & $\mathrm{C}$ & 1 & 0 & 0 & \\
\hline Burseraceae & Dacryodes nervosa (H.J.Lam) Leenh. & A & & & & \\
\hline Burseraceae & Dacryodes rostrata (Blume) H.J.Lam & $\mathrm{C}$ & 1 & 1 & 0 & \\
\hline Burseraceae & Dacryodes rugosa (Blume) H.J.Lam & $\mathrm{C}$ & & & & \\
\hline Burseraceae & Santiria apiculata A.W.Benn. & $\mathrm{C}$ & 1 & 1 & 0 & \\
\hline Burseraceae & Santiria conferta A.W.Benn. & $\mathrm{C}$ & & & & \\
\hline Burseraceae & Santiria griffithii (Hook.f.) Engl. & $\mathrm{C}$ & 1 & 1 & 0 & \\
\hline Burseraceae & Santiria laevigata Blume & $\mathrm{C}$ & 1 & 1 & 0 & \\
\hline
\end{tabular}


Appendix I. Continuation.

\begin{tabular}{|c|c|c|c|c|c|c|}
\hline Family & Accepted Name & Ref. & $\mathbf{P}$ & $\mathbf{O}$ & M & $\mathbf{N N}$ \\
\hline Burseraceae & Santiria rubiginosa Blume & $\mathrm{C}$ & 1 & 1 & 0 & \\
\hline Burseraceae & Santiria tomentosa Blume & $\mathrm{C}$ & & & & \\
\hline Burseraceae & Triomma malaccensis Hook.f. & $\mathrm{C}$ & 1 & 0 & 0 & \\
\hline Calophyllaceae & $\begin{array}{l}\text { Calophyllum costulatum M.R.Hend. \& Wyatt- } \\
\text { Sm. }\end{array}$ & $\mathrm{C} \dagger$ & & & & \\
\hline Calophyllaceae & Calophyllum ferrugineum Ridl. & $\mathrm{C}$ & 1 & 1 & 1 & \\
\hline Calophyllaceae & $\begin{array}{l}\text { Calophyllum lanigerum Miq. var. } \\
\text { austrocoriaceum (Whitmore) P.F.Stevens }\end{array}$ & & 1 & 1 & 0 & \\
\hline Calophyllaceae & Calophyllum macrocarpum Hook.f. & & 1 & 0 & 0 & \\
\hline Calophyllaceae & Calophyllum pulcherrimum Wall. ex Choisy & $\mathrm{C}$ & 1 & 1 & 1 & \\
\hline Calophyllaceae & Calophyllum rigidum Miq. & $\mathrm{C}$ & & & & \\
\hline Calophyllaceae & $\begin{array}{l}\text { Calophyllum rubiginosum M.R.Hend. \& Wyatt- } \\
\text { Sm. }\end{array}$ & $\mathrm{C}$ & 1 & 1 & 0 & \\
\hline Calophyllaceae & $\begin{array}{l}\text { Calophyllum rufigemmatum M.R.Hend. \& } \\
\text { Wyatt-Sm. }\end{array}$ & $\mathrm{C}$ & 1 & 1 & 0 & \\
\hline Calophyllaceae & Calophyllum sp. A & & 0 & 1 & 0 & \\
\hline Calophyllaceae & Calophyllum tetrapterum Miq. & $\mathrm{C}$ & 1 & 1 & 0 & \\
\hline Calophyllaceae & Calophyllum teysmannii Miq. & $\mathrm{C}$ & 1 & 1 & 0 & \\
\hline Calophyllaceae & $\begin{array}{l}\text { Calophyllum wallichianum Planch. \& Triana } \\
\text { var. incrassatum (M.R.Hend. \& Wyatt-Sm.) } \\
\text { P.F.Stevens }\end{array}$ & $\mathrm{C}$ & 1 & 1 & 0 & \\
\hline Cannabaceae & Gironniera nervosa Planch. & & 1 & 1 & 1 & \\
\hline Cannabaceae & Gironniera parvifolia Planch. & $\mathrm{C}$ & 1 & 1 & 0 & \\
\hline Cannabaceae & Gironniera subaequalis Planch. & $\mathrm{C}$ & 1 & 0 & 0 & \\
\hline Cannabaceae & Gironniera cf. hirta Ridl. & & 0 & 1 & 0 & \\
\hline Cannabaceae & Trema tomentosa (Roxb.) H.Hara & $\mathrm{C}$ & & & & \\
\hline
\end{tabular}


Appendix I. Continuation.

\begin{tabular}{|c|c|c|c|c|c|c|}
\hline Family & Accepted Name & Ref. & $\mathbf{P}$ & $\mathbf{O}$ & $\mathbf{M}$ & NN \\
\hline Capparaceae & $\begin{array}{l}\text { Capparis micracantha DC. subsp. korthalsiana } \\
\text { (Miq.) M.Jacobs }\end{array}$ & B & & & & \\
\hline Cardiopteridaceae & Gonocaryum gracile Miq. & & 0 & 1 & 0 & \\
\hline Celastraceae & Bhesa paniculata Arn. & $\mathrm{C}$ & 1 & 1 & 0 & \\
\hline Celastraceae & Bhesa robusta (Roxb.) Ding Hou & $\mathrm{C}$ & 0 & 1 & 1 & \\
\hline Celastraceae & Kokoona reflexa (M.A.Lawson) Ding Hou & $\mathrm{C}$ & 1 & 1 & 0 & \\
\hline Celastraceae & Lophopetalum multinervium Ridl. & $\mathrm{C}$ & & & & \\
\hline Celastraceae & Lophopetalum wightianum Arn. & & 1 & 0 & 0 & \\
\hline Celastraceae & Salacia grandiflora Kurz & $\mathrm{C}$ & 1 & 1 & 0 & \\
\hline Celastraceae & Salacia macrophylla Blume & $\mathrm{C}$ & 1 & 1 & 0 & \\
\hline Celastraceae & Salacia maingayi M.A.Lawson & & 1 & 1 & 0 & \\
\hline Celastraceae & Salacia viminea Wall. ex M.A.Lawson & $\mathrm{B}$ & 1 & 1 & 0 & \\
\hline Chloranthaceae & Chloranthus erectus (Buch.-Ham.) Verdc. & $\mathrm{C}$ & 1 & 1 & 0 & \\
\hline Chrysobalanaceae & Licania tomentosa (Benth.) Fritsch & $\mathrm{C}$ & & & & $\mathrm{NN}$ \\
\hline Chrysobalanaceae & Maranthes corymbosa Blume & $\mathrm{C}$ & 1 & 1 & 0 & \\
\hline Chrysobalanaceae & $\begin{array}{l}\text { Parastemon urophyllus (Wall. ex A.DC.) } \\
\text { A.DC. }\end{array}$ & $\mathrm{C}$ & & & & \\
\hline Chrysobalanaceae & Parinari oblongifolia Hook.f. & $\mathrm{B}$ & & & & \\
\hline Clusiaceae & Garcinia atroviridis Griff. ex T.Anderson & & 0 & 0 & 1 & \\
\hline Clusiaceae & Garcinia bancana Miq. & $\mathrm{C}$ & & & & \\
\hline Clusiaceae & Garcinia brevirostris Scheff. & $\mathrm{C}$ & 1 & 1 & 0 & \\
\hline Clusiaceae & Garcinia celebica $\mathrm{L}$. & $\mathrm{C}$ & & & & \\
\hline Clusiaceae & Garcinia forbesii King & $\mathrm{C}$ & 1 & 1 & 0 & \\
\hline Clusiaceae & Garcinia griffithii T.Anderson & $\mathrm{C}$ & 1 & 1 & 1 & \\
\hline
\end{tabular}


Appendix I. Continuation.

\begin{tabular}{|c|c|c|c|c|c|c|}
\hline Family & Accepted Name & Ref. & $\mathbf{P}$ & $\mathbf{O}$ & $\mathbf{M}$ & NN \\
\hline Clusiaceae & $\begin{array}{l}\text { Garcinia mangostana L. var. malaccensis } \\
\text { (Hook.f.) Nazre }\end{array}$ & & 0 & 1 & 0 & \\
\hline Clusiaceae & Garcinia nigrolineata Planch. ex T.Anderson & $\mathrm{C}$ & 1 & 1 & 0 & \\
\hline Clusiaceae & Garcinia parvifolia (Miq.) Miq. & $\mathrm{C}$ & 1 & 1 & 1 & \\
\hline Clusiaceae & Garcinia rostrata (Hassk.) Miq. & & 0 & 1 & 0 & \\
\hline Clusiaceae & Garcinia scortechinii King & $\mathrm{C}$ & 1 & 1 & 0 & \\
\hline Combretaceae & Combretum sundaicum Miq. & $\mathrm{C}$ & 1 & 1 & 0 & \\
\hline Combretaceae & Combretum tetralophum C.B.Clarke & & 1 & 0 & 0 & \\
\hline Combretaceae & Terminalia citrina (Gaertn.) Roxb. & A & & & & \\
\hline Combretaceae & Terminalia subspathulata King & $\mathrm{C}$ & 1 & 1 & 0 & \\
\hline Commelinaceae & Amischotolype gracilis (Ridl.) I.M.Turner & B & 1 & 1 & 0 & \\
\hline Commelinaceae & Amischotolype mollissima (Blume) Hassk. & $\mathrm{C}$ & & & & \\
\hline Connaraceae & Agelaea borneensis (Hook.f.) Merr. & $\mathrm{C}$ & 1 & 1 & 1 & \\
\hline Connaraceae & Agelaea macrophylla (Zoll.) Leenh. & $\mathrm{C}$ & 1 & 1 & 1 & \\
\hline Connaraceae & Agelaea sp. A & & 1 & 0 & 0 & \\
\hline Connaraceae & Cnestis palala (Lour.) Merr. & $\mathrm{C}$ & 0 & 1 & 0 & \\
\hline Connaraceae & Connarus ferrugineus Jack & $\mathrm{C}$ & 1 & 0 & 0 & \\
\hline Connaraceae & Connarus grandis Jack & & 1 & 0 & 0 & \\
\hline Connaraceae & Connarus monocarpus $\mathrm{L}$. & & 1 & 1 & 0 & \\
\hline Connaraceae & Connarus planchonianus G.Schellenb. & $\mathrm{C} \dagger$ & & & & \\
\hline Connaraceae & Connarus semidecandrus Jack & & 1 & 1 & 1 & \\
\hline Connaraceae & Ellipanthus tomentosus Kurz & $\mathrm{C}$ & & & & \\
\hline Connaraceae & Rourea acutipetala Miq. subsp. acutipetala & & 1 & 0 & 0 & \\
\hline Connaraceae & Rourea asplenifolia (G.Schellenb.) Jongkind & & 1 & 1 & 0 & \\
\hline
\end{tabular}


Appendix I. Continuation.

\begin{tabular}{|c|c|c|c|c|c|c|}
\hline Family & Accepted Name & Ref. & $\mathbf{P}$ & $\mathbf{O}$ & $\mathbf{M}$ & $\mathbf{N N}$ \\
\hline Connaraceae & Rourea fulgens Planch. & $\mathrm{B}$ & 1 & 1 & 0 & \\
\hline Connaraceae & Rourea mimosoides (Vahl) Planch. & & 1 & 1 & 0 & \\
\hline Connaraceae & Rourea minor (Gaertn.) Leenh. & $\mathrm{C}$ & 1 & 1 & 0 & \\
\hline Convolvulaceae & Argyreia ridleyi (Prain) Ooststr. & & 1 & 0 & 0 & \\
\hline Convolvulaceae & Erycibe leucoxyloides King ex Ridl. & $\mathrm{C}$ & & & & \\
\hline Convolvulaceae & Erycibe maingayi C.B.Clarke & $\mathrm{B} \dagger$ & & & & \\
\hline Convolvulaceae & Erycibe tomentosa Blume & $\mathrm{C}$ & 1 & 1 & 1 & \\
\hline Convolvulaceae & Merremia hederacea (Burm.f.) Hallier f. & $\mathrm{B}$ & & & & \\
\hline Convolvulaceae & Neuropeltis racemosa Wall. & $\mathrm{C}$ & 1 & 0 & 0 & \\
\hline Cornaceae & Alangium frutescens Zoll. \& Moritzi & & 1 & 1 & 0 & \\
\hline Cornaceae & $\begin{array}{l}\text { Alangium javanicum (Blume) Wangerin var. } \\
\text { ebenaceum (C.B.Clarke) Berhaman }\end{array}$ & $\mathrm{C}$ & 1 & 0 & 0 & \\
\hline Cornaceae & Alangium nobile (C.B.Clarke) Harms & $\mathrm{C}$ & 1 & 1 & 0 & \\
\hline Cornaceae & Alangium ridleyi King & $\mathrm{C}$ & 1 & 1 & 0 & \\
\hline Cornaceae & Alangium uniloculare (Griff.) King & $\mathrm{C}$ & 1 & 0 & 0 & \\
\hline Costaceae & Cheilocostus globosus (Blume) C.D.Specht & $\mathrm{C}$ & 1 & 0 & 0 & \\
\hline Crypteroniaceae & Crypteronia griffithii C.B.Clarke & $\mathrm{C}$ & 0 & 1 & 0 & \\
\hline Ctenolophonaceae & Ctenolophon parvifolius Oliv. & $\mathrm{C}$ & 1 & 0 & 0 & \\
\hline Cucurbitaceae & Trichosanthes quinquangulata A.Gray & $\mathrm{C}$ & 1 & 0 & 0 & \\
\hline Cucurbitaceae & Trichosanthes wawraei Cogn. & $\mathrm{C}$ & 1 & 1 & 0 & \\
\hline Cyperaceae & Carex cryptostachys Brongn. & $\mathrm{C}$ & & & & \\
\hline Cyperaceae & Cyperus cyperoides (L.) Kuntze & $\mathrm{C}$ & & & & \\
\hline Cyperaceae & Cyperus leptocarpus (F.Muell.) Bauters & $\mathrm{B}$ & & & & \\
\hline Cyperaceae & Diplacrum caricinum $\mathrm{R} . \mathrm{Br}$. & $\mathrm{B}$ & & & & \\
\hline
\end{tabular}


Appendix I. Continuation.

\begin{tabular}{|c|c|c|c|c|c|c|}
\hline Family & Accepted Name & Ref. & $\mathbf{P}$ & $\mathbf{O}$ & $\mathbf{M}$ & NN \\
\hline Cyperaceae & Fimbristylis acuminata Vahl & B & & & & \\
\hline Cyperaceae & $\begin{array}{l}\text { Fimbristylis dichotoma (L.) Vahl subsp. } \\
\text { dichotoma }\end{array}$ & B & & & & \\
\hline Cyperaceae & Fimbristylis leptoclada Benth. & B & & & & \\
\hline Cyperaceae & Fimbristylis obtusata (C.B.Clarke) Ridl. & B & & & & \\
\hline Cyperaceae & Fimbristylis pauciflora $\mathrm{R} . \mathrm{Br}$. & B & & & & \\
\hline Cyperaceae & Fuirena umbellata Rottb. & B & & & & \\
\hline Cyperaceae & Gahnia tristis Nees & B & 0 & 1 & 0 & \\
\hline Cyperaceae & Hypolytrum nemorum (Vahl) Spreng. & & 0 & 1 & 0 & \\
\hline Cyperaceae & Mapania cuspidata (Miq.) Uittien & $\mathrm{C}$ & 1 & 1 & 0 & \\
\hline Cyperaceae & Mapania kurzii C.B.Clarke & $\mathrm{C}$ & & & & \\
\hline Cyperaceae & Mapania longiflora C.B.Clarke & $\mathrm{B} \dagger$ & & & & \\
\hline Cyperaceae & Mapania lorea Uittien & $\mathrm{C} \dagger$ & & & & \\
\hline Cyperaceae & $\begin{array}{l}\text { Mapania palustris (Hassk. ex Steud.) Fern.- } \\
\text { Vill. }\end{array}$ & $\mathrm{C}$ & 0 & 1 & 0 & \\
\hline Cyperaceae & Mapania squamata (Kurz) C.B.Clarke & $\mathrm{C} \dagger$ & & & & \\
\hline Cyperaceae & Mapania wallichii C.B.Clarke & $\mathrm{C} \dagger$ & & & & \\
\hline Cyperaceae & Mapania sp. & & 1 & 0 & 0 & \\
\hline Cyperaceae & Scleria biflora Roxb. subsp. biflora & B & & & & \\
\hline Cyperaceae & Scleria corymbosa Roxb. & $\mathrm{B} \dagger$ & & & & \\
\hline Cyperaceae & Scleria levis Retz. & & 0 & 1 & 0 & \\
\hline Cyperaceae & Scleria purpurascens Steud. & B & & & & \\
\hline Cyperaceae & Scleria sumatrensis Retz. & B & & & & \\
\hline Cyperaceae & Scleria terrestris (L.) Fassett & B & 0 & 1 & 0 & \\
\hline Dilleniaceae & Dillenia excelsa (Jack) Gilg & $\mathrm{C}$ & 1 & 0 & 0 & \\
\hline
\end{tabular}


Appendix I. Continuation.

\begin{tabular}{|c|c|c|c|c|c|c|}
\hline Family & Accepted Name & Ref. & $\mathbf{P}$ & $\mathbf{O}$ & $\mathbf{M}$ & $\mathbf{N N}$ \\
\hline Dilleniaceae & $\begin{array}{l}\text { Dillenia grandifolia Wall. ex Hook.f. \& } \\
\text { Thomson }\end{array}$ & $\mathrm{C}$ & 0 & 1 & 0 & \\
\hline Dilleniaceae & Dillenia pulchella (Jack) Gilg & $\mathrm{C}$ & & & & \\
\hline Dilleniaceae & Dillenia sp. & & 0 & 1 & 0 & \\
\hline Dilleniaceae & $\begin{array}{l}\text { Dillenia suffruticosa (Griff. ex Hook.f. \& } \\
\text { Thomson) Martelli }\end{array}$ & $\mathrm{C}$ & 1 & 1 & 1 & \\
\hline Dilleniaceae & Tetracera akara (Burm.f.) Merr. & & 0 & 1 & 0 & \\
\hline Dilleniaceae & Tetracera fagifolia Blume & & 1 & 1 & 1 & \\
\hline Dilleniaceae & Tetracera indica (Christm. \& Panz.) Merr. & $\mathrm{C}$ & 0 & 1 & 0 & \\
\hline Dilleniaceae & $\begin{array}{l}\text { Tetracera macrophylla Wall. ex Hook.f. \& } \\
\text { Thomson }\end{array}$ & & 1 & 1 & 0 & \\
\hline Dilleniaceae & Tetracera sp. A & & 0 & 1 & 0 & \\
\hline Dilleniaceae & Tetracera sp. B & & 1 & 1 & 0 & \\
\hline Dioscoreaceae & Dioscorea bulbifera $\mathrm{L}$. & & 0 & 1 & 1 & \\
\hline Dioscoreaceae & Dioscorea kingii R.Knuth & A & 0 & 1 & 0 & \\
\hline Dioscoreaceae & Dioscorea laurifolia Wall. ex Hook.f. & $\mathrm{C}$ & 1 & 1 & 0 & \\
\hline Dioscoreaceae & $\begin{array}{l}\text { Dioscorea orbiculata Hook.f. var. tenuifolia } \\
\text { (Ridl.) Thapyai }\end{array}$ & $\mathrm{C}$ & 1 & 1 & 0 & \\
\hline Dioscoreaceae & Dioscorea polyclados Hook.f. & $\mathrm{C}$ & 1 & 1 & 0 & \\
\hline Dioscoreaceae & Dioscorea prainiana R.Knuth & $\mathrm{C}$ & & & & \\
\hline Dioscoreaceae & Dioscorea pyrifolia Kunth & $\mathrm{C}$ & & & & \\
\hline Dioscoreaceae & Dioscorea sansibarensis $\mathrm{Pax}$ & & 0 & 0 & 1 & NN \\
\hline Dioscoreaceae & Dioscorea sp. & & 1 & 0 & 0 & \\
\hline Dioscoreaceae & Tacca integrifolia Ker Gawl. & $\mathrm{C}$ & 1 & 1 & 1 & \\
\hline Dioscoreaceae & Thismia aseroe Becc. & $\mathrm{C} \dagger$ & & & & \\
\hline Dipterocarpaceae & Anisoptera megistocarpa Slooten & $\mathrm{C}$ & 1 & 0 & 0 & \\
\hline
\end{tabular}


Appendix I. Continuation.

\begin{tabular}{|c|c|c|c|c|c|c|}
\hline Family & Accepted Name & Ref. & $\mathbf{P}$ & $\mathbf{O}$ & M & $\mathbf{N N}$ \\
\hline Dipterocarpaceae & $\begin{array}{l}\text { Dipterocarpus caudatus Foxw. subsp. } \\
\text { penangianus (Foxw.) P.S.Ashton }\end{array}$ & $\mathrm{C}$ & 1 & 1 & 0 & \\
\hline Dipterocarpaceae & Dipterocarpus cornutus Dyer & $\mathrm{C}$ & & & & \\
\hline Dipterocarpaceae & Hopea ferruginea Parijs & A & & & & \\
\hline Dipterocarpaceae & Hopea griffithii Kurz & $\mathrm{C}$ & 1 & 0 & 0 & \\
\hline Dipterocarpaceae & Hopea mengarawan Miq. & $\mathrm{C}$ & 1 & 1 & 0 & \\
\hline Dipterocarpaceae & Hopea sangal Korth. & & 1 & 0 & 0 & \\
\hline Dipterocarpaceae & Shorea curtisii Dyer ex King subsp. curtisii & $\mathrm{C}$ & 1 & 1 & 0 & \\
\hline Dipterocarpaceae & Shorea gibbosa Brandis & & 1 & 1 & 0 & \\
\hline Dipterocarpaceae & Shorea gratissima (Wall. ex Kurz) Dyer & $\mathrm{C}$ & & & & \\
\hline Dipterocarpaceae & Shorea leprosula Miq. & $\mathrm{C}$ & 1 & 0 & 0 & \\
\hline Dipterocarpaceae & Shorea macroptera Dyer subsp. macroptera & $\mathrm{C}$ & 1 & 0 & 0 & \\
\hline Dipterocarpaceae & Shorea ochrophloia Strugnell ex Symington & $\mathrm{C}$ & 1 & 0 & 0 & \\
\hline Dipterocarpaceae & Shorea parvifolia Dyer & $\mathrm{C}$ & 0 & 1 & 0 & \\
\hline Dipterocarpaceae & Shorea pauciflora King & $\mathrm{C}$ & 1 & 0 & 0 & \\
\hline Dipterocarpaceae & Vatica maingayi Dyer & $\mathrm{C}$ & & & & \\
\hline Dipterocarpaceae & $\begin{array}{l}\text { Vatica odorata (Griff.) Symington subsp. } \\
\text { odorata }\end{array}$ & A & 1 & 0 & 0 & \\
\hline Dipterocarpaceae & Vatica pauciflora Blume & $\mathrm{C}$ & 1 & 0 & 0 & \\
\hline Dipterocarpaceae & Vatica ridleyana Brandis & $\mathrm{C}$ & 0 & 1 & 0 & \\
\hline Dipterocarpaceae & Vatica sp. & & 0 & 1 & 0 & \\
\hline Ebenaceae & Diospyros argentea Griff. & $\mathrm{C}$ & & & & \\
\hline Ebenaceae & Diospyros buxifolia (Blume) Hiern & $\mathrm{C}$ & 1 & 1 & 0 & \\
\hline Ebenaceae & Diospyros clavigera C.B.Clarke & $\mathrm{C}$ & 0 & 1 & 0 & \\
\hline Ebenaceae & Diospyros confusa Bakh. & & 1 & 1 & 0 & \\
\hline
\end{tabular}


Appendix I. Continuation.

\begin{tabular}{|c|c|c|c|c|c|c|}
\hline Family & Accepted Name & Ref. & $\mathbf{P}$ & $\mathbf{O}$ & $\mathbf{M}$ & NN \\
\hline Ebenaceae & Diospyros coriacea Hiern & $\mathrm{C}$ & 1 & 0 & 0 & \\
\hline Ebenaceae & Diospyros diepenhorstii Miq. & $\mathrm{C}$ & 1 & 0 & 0 & \\
\hline Ebenaceae & Diospyros lanceifolia Roxb. & $\mathrm{C}$ & 1 & 1 & 0 & \\
\hline Ebenaceae & Diospyros maingayi (Hiern) Bakh. & $\mathrm{C}$ & & & & \\
\hline Ebenaceae & $\begin{array}{l}\text { Diospyros pilosanthera Blanco var. oblonga } \\
\text { (Wall. ex G.Don) } \mathrm{Ng}\end{array}$ & $\mathrm{C}$ & 1 & 1 & 0 & \\
\hline Ebenaceae & Diospyros styraciformis King \& Gamble & $\mathrm{C}$ & 1 & 0 & 0 & \\
\hline Ebenaceae & Diospyros sumatrana Miq. & & 1 & 1 & 0 & \\
\hline Ebenaceae & Diospyros venosa Wall. ex A.DC. & & 1 & 1 & 0 & \\
\hline Elaeocarpaceae & Elaeocarpus ferrugineus (Jack) Steud. & $\mathrm{C}$ & 1 & 1 & 0 & \\
\hline Elaeocarpaceae & Elaeocarpus floribundus Blume & $\mathrm{C}$ & 1 & 0 & 0 & \\
\hline Elaeocarpaceae & Elaeocarpus macrocerus (Turcz.) Merr. & $\mathrm{C}$ & & & & \\
\hline Elaeocarpaceae & Elaeocarpus mastersii King & $\mathrm{C}$ & 1 & 1 & 1 & \\
\hline Elaeocarpaceae & Elaeocarpus nitidus Jack & $\mathrm{C}$ & 1 & 1 & 0 & \\
\hline Elaeocarpaceae & $\begin{array}{l}\text { Elaeocarpus obtusus Blume subsp. apiculatus } \\
\text { (Mast.) Coode. }\end{array}$ & $\mathrm{C}$ & & & & $\mathrm{NN}$ \\
\hline Elaeocarpaceae & Elaeocarpus palembanicus (Miq.) Corner & B & 1 & 0 & 0 & \\
\hline Elaeocarpaceae & Elaeocarpus petiolatus (Jack) Wall. & $\mathrm{C}$ & 1 & 1 & 0 & \\
\hline Elaeocarpaceae & Elaeocarpus polystachyus Wall. ex Müll.Berol. & $\mathrm{C}$ & 1 & 1 & 0 & \\
\hline Elaeocarpaceae & Elaeocarpus salicifolius King & $\mathrm{C}$ & 0 & 1 & 1 & \\
\hline Elaeocarpaceae & Elaeocarpus stipularis Blume & $\mathrm{C}$ & 0 & 1 & 0 & \\
\hline Elaeocarpaceae & Sloanea javanica (Miq.) Szyszył. ex K.Schum. & $\mathrm{C}$ & 1 & 0 & 0 & \\
\hline Ericaceae & Rhododendron longiflorum Lindl. & $\mathrm{C} \dagger$ & & & & \\
\hline Escalloniaceae & Polyosma kingiana Schltr. & $\mathrm{C}$ & & & & \\
\hline Euphorbiaceae & Acalypha siamensis Oliv. ex Gage & & 1 & 0 & 0 & $\mathrm{NN}$ \\
\hline
\end{tabular}


Appendix I. Continuation.

\begin{tabular}{|c|c|c|c|c|c|c|}
\hline Family & Accepted Name & Ref. & $\mathbf{P}$ & $\mathbf{O}$ & M & $\mathbf{N N}$ \\
\hline Euphorbiaceae & Agrostistachys borneensis Becc. & $\mathrm{C}$ & 1 & 1 & 0 & \\
\hline Euphorbiaceae & Alchornea tiliifolia (Benth.) Müll.Arg. & $\mathrm{C}$ & & & & \\
\hline Euphorbiaceae & Blumeodendron tokbrai (Blume) Kurz & $\mathrm{C}$ & 1 & 1 & 0 & \\
\hline Euphorbiaceae & Cheilosa montana Blume & & 1 & 0 & 0 & \\
\hline Euphorbiaceae & Claoxylon indicum (Reinw. ex Blume) Hassk. & & 1 & 1 & 1 & \\
\hline Euphorbiaceae & Claoxylon longifolium (Blume) Endl. ex Hassk. & $\mathrm{C}$ & 1 & 0 & 0 & \\
\hline Euphorbiaceae & Croton caudatus Geiseler & $\mathrm{C}$ & 1 & 1 & 0 & \\
\hline Euphorbiaceae & Croton oblongus Burm.f. & $\mathrm{C}$ & 1 & 1 & 0 & \\
\hline Euphorbiaceae & Endospermum diadenum (Miq.) Airy Shaw & $\mathrm{C}$ & 1 & 1 & 0 & \\
\hline Euphorbiaceae & $\begin{array}{l}\text { Hancea penangensis (Müll.Arg.) S.E.C.Sierra, } \\
\text { Kulju \& Welzen }\end{array}$ & $\mathrm{C}$ & 1 & 1 & 0 & \\
\hline Euphorbiaceae & $\begin{array}{l}\text { Hevea brasiliensis (Willd. ex A.Juss.) } \\
\text { Müll.Arg. }\end{array}$ & & 1 & 0 & 1 & $\mathrm{NN}$ \\
\hline Euphorbiaceae & Koilodepas longifolium Hook.f. & $\mathrm{C}$ & 1 & 1 & 0 & \\
\hline Euphorbiaceae & Macaranga bancana (Miq.) Müll.Arg. & $\mathrm{C}$ & 1 & 1 & 1 & \\
\hline Euphorbiaceae & Macaranga conifera (Zoll.) Müll.Arg. & $\mathrm{C}$ & 1 & 1 & 0 & \\
\hline Euphorbiaceae & $\begin{array}{l}\text { Macaranga gigantea (Rchb.f. \& Zoll.) } \\
\text { Müll.Arg. }\end{array}$ & $\mathrm{C}$ & 0 & 0 & 1 & \\
\hline Euphorbiaceae & Macaranga griffithiana Müll.Arg. & $\mathrm{C}$ & & & & \\
\hline Euphorbiaceae & Macaranga heynei I.M.Johnst. & $\mathrm{C}$ & 1 & 1 & 0 & \\
\hline Euphorbiaceae & Macaranga hullettii King ex Hook.f. & & 0 & 1 & 0 & \\
\hline Euphorbiaceae & $\begin{array}{l}\text { Macaranga hypoleuca (Rchb.f. \& Zoll.) } \\
\text { Müll.Arg. }\end{array}$ & $\mathrm{C}$ & & & & \\
\hline Euphorbiaceae & Macaranga lowii King ex Hook.f. & $\mathrm{C}$ & 1 & 1 & 0 & \\
\hline Euphorbiaceae & Macaranga recurvata Gage & & 1 & 0 & 1 & \\
\hline
\end{tabular}


Appendix I. Continuation.

\begin{tabular}{|c|c|c|c|c|c|c|}
\hline Family & Accepted Name & Ref. & $\mathbf{P}$ & $\mathbf{O}$ & $\mathbf{M}$ & $\mathbf{N N}$ \\
\hline Euphorbiaceae & $\begin{array}{l}\text { Macaranga trichocarpa (Rchb.f. \& Zoll.) } \\
\text { Müll.Arg. }\end{array}$ & $\mathrm{C}$ & 1 & 1 & 0 & \\
\hline Euphorbiaceae & Mallotus macrostachyus (Miq.) Müll.Arg. & $\mathrm{C} \dagger$ & & & & \\
\hline Euphorbiaceae & Mallotus paniculatus (Lam.) Müll.Arg. & $\mathrm{C}$ & & & & \\
\hline Euphorbiaceae & $\begin{array}{l}\text { Neoscortechinia kingii (Hook.f.) Pax \& } \\
\text { K.Hoffm. }\end{array}$ & $\mathrm{C}$ & 0 & 1 & 0 & \\
\hline Euphorbiaceae & Neoscortechinia philippinensis (Merr.) Welzen & A & & & & \\
\hline Euphorbiaceae & Paracroton pendulus (Hassk.) Miq. & $\mathrm{C}$ & & & & \\
\hline Euphorbiaceae & $\begin{array}{l}\text { Pimelodendron griffithianum (Müll.Arg.) } \\
\text { Benth. }\end{array}$ & $\mathrm{C}$ & 1 & 1 & 0 & \\
\hline Euphorbiaceae & Ptychopyxis caput-medusae (Hook.f.) Ridl. & $\mathrm{C}$ & 1 & 1 & 0 & \\
\hline Euphorbiaceae & Suregada multiflora (A.Juss.) Baill. & $\mathrm{C}$ & & & & \\
\hline Euphorbiaceae & Triadica cochinchinensis Lour. & $\mathrm{C}$ & & & & \\
\hline Euphorbiaceae & Trigonostemon heteranthus Wight & $\mathrm{C}$ & 1 & 1 & 0 & \\
\hline Euphorbiaceae & (unidentified) & & 1 & 0 & 0 & \\
\hline Fabaceae & Adenanthera malayana Kosterm. & $\mathrm{C}$ & 1 & 1 & 0 & \\
\hline Fabaceae & Adenanthera pavonina $\mathrm{L}$. & $\mathrm{C}$ & 1 & 1 & 0 & $\mathrm{NN}$ \\
\hline Fabaceae & Aganope thyrsiflora (Benth.) Polhill & $\mathrm{C}$ & 1 & 1 & 0 & \\
\hline Fabaceae & Albizia splendens Miq. & $\mathrm{C}$ & 1 & 1 & 0 & \\
\hline Fabaceae & Archidendron clypearia (Jack) I.C.Nielsen & $\mathrm{C}$ & 1 & 1 & 0 & \\
\hline Fabaceae & Archidendron contortum (Mart.) I.C.Nielsen & $\mathrm{C}$ & 1 & 1 & 0 & \\
\hline Fabaceae & Archidendron ellipticum (Blume) I.C.Nielsen & $\mathrm{C}$ & 1 & 1 & 0 & \\
\hline Fabaceae & Archidendron jiringa (Jack) I.C.Nielsen & & 1 & 1 & 1 & \\
\hline Fabaceae & $\begin{array}{l}\text { Archidendron microcarpum (Benth.) } \\
\text { I.C.Nielsen }\end{array}$ & $\mathrm{C}$ & & & & \\
\hline Fabaceae & Callerya atropurpurea (Wall.) Schot & $\mathrm{C}$ & 1 & 1 & 0 & $\mathrm{NN}$ \\
\hline
\end{tabular}


Appendix I. Continuation.

\begin{tabular}{|c|c|c|c|c|c|c|}
\hline Family & Accepted Name & Ref. & $\mathbf{P}$ & $\mathbf{O}$ & $\mathbf{M}$ & NN \\
\hline Fabaceae & Callerya eriantha (Benth.) Schot & & 0 & 1 & 0 & \\
\hline Fabaceae & Dalbergia junghuhnii Benth. & $\mathrm{C}$ & & & & \\
\hline Fabaceae & Dalbergia parviflora Roxb. & $\mathrm{C}$ & & & & \\
\hline Fabaceae & Dalbergia rostrata Hassk. & $\mathrm{C}$ & 1 & 1 & 0 & \\
\hline Fabaceae & Dalbergia velutina Benth. & & 1 & 1 & 0 & \\
\hline Fabaceae & Derris amoena Benth. & $\mathrm{C}$ & 1 & 1 & 0 & \\
\hline Fabaceae & Derris elliptica (Wall.) Benth. & & 0 & 0 & 1 & \\
\hline Fabaceae & Dialium indum L. var. indum & $\mathrm{C}$ & 1 & 1 & 0 & \\
\hline Fabaceae & Dialium platysepalum Baker & $\mathrm{C}$ & 1 & 1 & 0 & \\
\hline Fabaceae & Entada spiralis Ridl. & $\mathrm{C}$ & & & & \\
\hline Fabaceae & $\begin{array}{l}\text { Falcataria moluccana (Miq.) Barneby \& } \\
\text { J.W.Grimes }\end{array}$ & $\mathrm{C}$ & 0 & 1 & 0 & NN \\
\hline Fabaceae & Intsia bijuga (Colebr.) Kuntze & $\mathrm{C}$ & & & & \\
\hline Fabaceae & Intsia palembanica Miq. & B & & & & \\
\hline Fabaceae & Koompassia malaccensis Maingay ex Benth. & $\mathrm{C}$ & 1 & 1 & 0 & \\
\hline Fabaceae & Kunstleria ridleyi Prain & $\mathrm{C}$ & 1 & 1 & 1 & \\
\hline Fabaceae & $\begin{array}{l}\text { Mezoneuron sumatranum (Roxb.) Wight \& } \\
\text { Arn. ex Miq. }\end{array}$ & $\mathrm{C}$ & & & & \\
\hline Fabaceae & Ormosia bancana (Miq.) Merr. & $\mathrm{C}$ & & & & \\
\hline Fabaceae & Parkia speciosa Hassk. & $\mathrm{C}$ & 1 & 1 & 0 & \\
\hline Fabaceae & $\begin{array}{l}\text { Phanera semibifida (Roxb.) Benth. var. } \\
\text { semibifida }\end{array}$ & $\mathrm{C}$ & 1 & 1 & 0 & \\
\hline Fabaceae & $\begin{array}{l}\text { Senegalia kekapur (I.C.Nielsen) Maslin, Seigler } \\
\text { \& Ebinger }\end{array}$ & $\mathrm{C}$ & 1 & 0 & 0 & \\
\hline Fabaceae & Sindora echinocalyx Prain & A & 1 & 0 & 0 & \\
\hline Fabaceae & Sindora wallichii Benth. & $\mathrm{C}$ & 1 & 0 & 0 & \\
\hline
\end{tabular}


Appendix I. Continuation.

\begin{tabular}{|c|c|c|c|c|c|c|}
\hline Family & Accepted Name & Ref. & $\mathbf{P}$ & $\mathbf{O}$ & $\mathbf{M}$ & $\mathbf{N N}$ \\
\hline Fabaceae & $\begin{array}{l}\text { Spatholobus ferrugineus (Zoll. \& Moritzi) } \\
\text { Benth. }\end{array}$ & $\mathrm{C}$ & 1 & 1 & 1 & \\
\hline Fabaceae & Spatholobus maingayi Prain & $\mathrm{C}$ & 1 & 1 & 0 & \\
\hline Fabaceae & Spatholobus cf. ridleyi Prain & & 1 & 1 & 0 & \\
\hline Fabaceae & Spatholobus sp. A & & 1 & 0 & 0 & \\
\hline Fagaceae & Castanopsis lucida (Nees) Soepadmo & $\mathrm{C}$ & 1 & 1 & 0 & \\
\hline Fagaceae & Castanopsis malaccensis Gamble & $\mathrm{C}$ & & & & \\
\hline Fagaceae & Castanopsis megacarpa Gamble & $\mathrm{C}$ & 1 & 0 & 0 & \\
\hline Fagaceae & Castanopsis nephelioides King ex Hook.f. & $\mathrm{C}$ & & & & \\
\hline Fagaceae & Castanopsis wallichii King ex Hook.f. & $\mathrm{C}$ & 1 & 0 & 0 & \\
\hline Fagaceae & $\begin{array}{l}\text { Lithocarpus cantleyanus (King ex Hook.f.) } \\
\text { Rehder }\end{array}$ & $\mathrm{C}$ & 0 & 1 & 0 & \\
\hline Fagaceae & Lithocarpus conocarpus (Oudem.) Rehder & $\mathrm{C}$ & 1 & 1 & 0 & \\
\hline Fagaceae & $\begin{array}{l}\text { Lithocarpus elegans (Blume) Hatus. ex } \\
\text { Soepadmo }\end{array}$ & $\mathrm{C}$ & 0 & 1 & 0 & \\
\hline Fagaceae & Lithocarpus ewyckii (Korth.) Rehder & $\mathrm{C}$ & 1 & 1 & 0 & \\
\hline Fagaceae & Lithocarpus cf. gracilis (Korth.) Soepadmo & & 1 & 0 & 0 & \\
\hline Fagaceae & Lithocarpus hystrix (Korth.) Rehder & $\mathrm{C}$ & & & & \\
\hline Fagaceae & Lithocarpus lucidus (Roxb.) Rehder & $\mathrm{C}$ & 1 & 0 & 0 & \\
\hline Fagaceae & Quercus argentata Korth. & $\mathrm{C}$ & 1 & 0 & 0 & \\
\hline Fagaceae & Quercus sp. A & & 1 & 0 & 0 & \\
\hline Flagellariaceae & Flagellaria indica $\mathrm{L}$. & & 0 & 1 & 0 & \\
\hline Gentianaceae & Cyrtophyllum fragrans (Roxb.) DC. & & 0 & 1 & 0 & \\
\hline Gentianaceae & Fagraea ridleyi King \& Gamble & $\mathrm{C} \dagger$ & & & & \\
\hline Gentianaceae & $\begin{array}{l}\text { Utania volubilis (Wall.) Sugumaran var. } \\
\text { volubilis }\end{array}$ & $\mathrm{C}$ & & & & \\
\hline
\end{tabular}


Appendix I. Continuation.

\begin{tabular}{|c|c|c|c|c|c|c|}
\hline Family & Accepted Name & Ref. & $\mathbf{P}$ & $\mathbf{O}$ & $\mathbf{M}$ & NN \\
\hline Gesneriaceae & Aeschynanthus pulcher (Blume) G.Don & $\mathrm{C} \dagger$ & & & & \\
\hline Gesneriaceae & Aeschynanthus radicans Jack & $\mathrm{B} \dagger$ & & & & \\
\hline Gesneriaceae & Codonoboea platypus (C.B.Clarke) C.L.Lim & $\mathrm{C}$ & 1 & 1 & 0 & \\
\hline Gesneriaceae & Cyrtandra pendula Blume & $\mathrm{C}$ & 1 & 0 & 0 & \\
\hline Hanguanaceae & Hanguana corneri Škorničk. \& P.C.Boyce & A & & & & \\
\hline Hanguanaceae & Hanguana neglecta Škorničk. \& Niissalo & A & 0 & 1 & 0 & \\
\hline Hanguanaceae & $\begin{array}{l}\text { Hanguana nitens Siti Nurfazilah, Mohd Fahmi, } \\
\text { Sofiman Othman \& P.C.Boyce }\end{array}$ & $\mathrm{C}$ & & & & \\
\hline Hanguanaceae & Hanguana rubinea Škorničk. \& P.C.Boyce & A & 1 & 0 & 0 & \\
\hline Hanguanaceae & Hanguana triangulata Škorničk. \& P.C.Boyce & $\mathrm{A}$ & & & & \\
\hline Hanguanaceae & Hanguana sp. & & 0 & 1 & 0 & \\
\hline Heliconiaceae & Heliconia psittacorum L.f. & & 0 & 0 & 1 & $\mathrm{NN}$ \\
\hline Hernandiaceae & Illigera trifoliata (Griff.) Dunn & $\mathrm{C} \dagger$ & & & & \\
\hline Hypericaceae & Cratoxylum arborescens (Vahl) Blume & $\mathrm{C}$ & & & & \\
\hline Hypericaceae & Cratoxylum cochinchinense (Lour.) Blume & $\mathrm{C}$ & 1 & 1 & 0 & \\
\hline Hypericaceae & Cratoxylum formosum (Jack) Dyer & $\mathrm{C}$ & 1 & 1 & 0 & \\
\hline Hypericaceae & Cratoxylum maingayi Dyer & & 1 & 1 & 0 & \\
\hline Hypericaceae & Cratoxylum sp. & & 0 & 1 & 0 & \\
\hline Hypoxidaceae & $\begin{array}{l}\text { Molineria latifolia (Dryand. ex W.T.Aiton) } \\
\text { Herb. ex Kurz var. latifolia }\end{array}$ & $\mathrm{C}$ & 1 & 1 & 0 & \\
\hline Icacinaceae & Iodes cirrhosa Turcz. & & 1 & 0 & 0 & \\
\hline Icacinaceae & Iodes ovalis Blume & & 1 & 1 & 0 & \\
\hline Icacinaceae & Phytocrene bracteata Wall. & $\mathrm{C}$ & 1 & 1 & 0 & \\
\hline Irvingiaceae & Irvingia malayana Oliv. ex A.W.Benn. & $\mathrm{C}$ & 1 & 0 & 0 & \\
\hline Ixonanthaceae & Ixonanthes icosandra Jack & $\mathrm{C}$ & 1 & 1 & 0 & \\
\hline
\end{tabular}


Appendix I. Continuation.

\begin{tabular}{|c|c|c|c|c|c|c|}
\hline Family & Accepted Name & Ref. & $\mathbf{P}$ & $\mathbf{O}$ & $\mathbf{M}$ & $\mathbf{N N}$ \\
\hline Ixonanthaceae & Ixonanthes reticulata Jack & $\mathrm{C}$ & 1 & 1 & 0 & \\
\hline Lamiaceae & Callicarpa longifolia Lam. & $\mathrm{B}$ & & & & \\
\hline Lamiaceae & Clerodendrum deflexum Wall. & $\mathrm{C}$ & 1 & 1 & 0 & \\
\hline Lamiaceae & Clerodendrum disparifolium Blume & $\mathrm{C}$ & 1 & 1 & 1 & \\
\hline Lamiaceae & Clerodendrum villosum Blume & $\mathrm{C}$ & & & & \\
\hline Lamiaceae & $\begin{array}{l}\text { Teijsmanniodendron coriaceum (C.B.Clarke) } \\
\text { Kosterm. }\end{array}$ & $\mathrm{C}$ & & & & \\
\hline Lamiaceae & Vitex negundo L. & $\mathrm{B}$ & & & & \\
\hline Lamiaceae & Vitex pinnata $\mathrm{L}$. & $\mathrm{C}$ & 1 & 0 & 1 & \\
\hline Lamiaceae & Vitex vestita Wall. ex Schauer & $\mathrm{C}$ & 1 & 0 & 0 & \\
\hline Lauraceae & Actinodaphne glomerata (Blume) Nees & $\mathrm{C}$ & & & & \\
\hline Lauraceae & Actinodaphne malaccensis Hook.f. & $\mathrm{C}$ & 1 & 1 & 0 & \\
\hline Lauraceae & Actinodaphne pruinosa Nees & & 1 & 0 & 0 & \\
\hline Lauraceae & Alseodaphne bancana Miq. & $\mathrm{C}$ & 1 & 0 & 0 & \\
\hline Lauraceae & Alseodaphne nigrescens (Gamble) Kosterm. & & 1 & 0 & 0 & \\
\hline Lauraceae & Alseodaphne sp. A & & 0 & 1 & 0 & \\
\hline Lauraceae & Beilschmiedia kunstleri Gamble & $\mathrm{C}$ & 1 & 0 & 0 & \\
\hline Lauraceae & Beilschmiedia madang Blume & $\mathrm{C}$ & 1 & 1 & 0 & \\
\hline Lauraceae & Cinnamomum iners Reinw. ex Blume & & 1 & 1 & 1 & \\
\hline Lauraceae & Cinnamomum javanicum Blume & $\mathrm{B}$ & & & & \\
\hline Lauraceae & Cryptocarya ferrea Blume & $\mathrm{C}$ & & & & \\
\hline Lauraceae & Cryptocarya griffithiana Wight & $\mathrm{C}$ & 1 & 1 & 0 & \\
\hline Lauraceae & Cryptocarya impressa Miq. & $\mathrm{C}$ & & & & \\
\hline Lauraceae & Cryptocarya cf. kurzii Hook.f. & & 0 & 1 & 0 & \\
\hline
\end{tabular}


Appendix I. Continuation.

\begin{tabular}{|c|c|c|c|c|c|c|}
\hline Family & Accepted Name & Ref. & $\mathbf{P}$ & $\mathbf{O}$ & $\mathbf{M}$ & NN \\
\hline Lauraceae & Cryptocarya nitens (Blume) Koord. \& Valeton & A & 1 & 0 & 0 & \\
\hline Lauraceae & Cryptocarya rugulosa Hook.f. & $\mathrm{C}$ & 1 & 1 & 0 & \\
\hline Lauraceae & Dehaasia cuneata (Blume) Blume & A & 1 & 0 & 0 & \\
\hline Lauraceae & Endiandra maingayi Hook.f. & A & & & & \\
\hline Lauraceae & Lindera lucida (Blume) Boerl. & $\mathrm{C}$ & 1 & 0 & 0 & \\
\hline Lauraceae & Litsea accedens (Blume) Boerl. & $\mathrm{C}$ & 1 & 1 & 0 & \\
\hline Lauraceae & Litsea castanea Hook.f. & $\mathrm{C}$ & 1 & 1 & 0 & \\
\hline Lauraceae & Litsea costalis (Nees) Kosterm. & & 1 & 1 & 0 & \\
\hline Lauraceae & Litsea costata (Blume) Boerl. & $\mathrm{C}$ & & & & \\
\hline Lauraceae & Litsea elliptica Blume & $\mathrm{C}$ & 1 & 1 & 0 & \\
\hline Lauraceae & Litsea ferruginea Blume & $\mathrm{C}$ & & & & \\
\hline Lauraceae & Litsea firma Hook.f. & $\mathrm{C}$ & 1 & 1 & 0 & \\
\hline Lauraceae & Litsea grandis Hook.f. & $\mathrm{C}$ & 1 & 1 & 0 & \\
\hline Lauraceae & Litsea machilifolia Gamble & $\mathrm{C}$ & & & & \\
\hline Lauraceae & Litsea ridleyi Gamble & $\mathrm{C}$ & 1 & 1 & 0 & \\
\hline Lauraceae & Litsea robusta Blume & & 1 & 0 & 0 & \\
\hline Lauraceae & Litsea umbellata (Lour.) Merr. & $\mathrm{C}$ & 1 & 1 & 0 & \\
\hline Lauraceae & Neolitsea cassia (L.) Kosterm. & $\mathrm{C}$ & 1 & 0 & 0 & \\
\hline Lauraceae & Nothaphoebe umbelliflora (Blume) Blume & $\mathrm{C}$ & 1 & 1 & 1 & \\
\hline Lecythidaceae & Barringtonia racemosa (L.) Spreng. & $\mathrm{C}$ & & & & \\
\hline Lentibulariaceae & Utricularia aurea Lour. & B & & & & \\
\hline Linaceae & Indorouchera griffithiana (Planch.) Hallier $\mathrm{f}$. & $\mathrm{C}$ & 1 & 0 & 1 & \\
\hline Linderniaceae & Lindernia crustacea (L.) F.Muell. & $\mathrm{B}$ & & & & \\
\hline
\end{tabular}


Appendix I. Continuation.

\begin{tabular}{|c|c|c|c|c|c|c|}
\hline Family & Accepted Name & Ref. & $\mathbf{P}$ & $\mathbf{O}$ & $\mathbf{M}$ & NN \\
\hline Loganiaceae & Strychnos axillaris Colebr. & $\mathrm{C}$ & 1 & 1 & 0 & \\
\hline Loganiaceae & Strychnos ignatii P.J.Bergius & & 1 & 1 & 0 & \\
\hline Loganiaceae & Strychnos maingayi C.B.Clarke & $\mathrm{C}$ & 1 & 0 & 0 & \\
\hline Loranthaceae & Amylotheca duthieana (King) Danser & $\mathrm{C} \dagger$ & & & & \\
\hline Loranthaceae & Barathranthus axanthus (Korth.) Miq. & $\mathrm{C} \dagger$ & & & & \\
\hline Loranthaceae & Dendrophthoe pentandra (L.) Miq. & $\mathrm{C}$ & & & & \\
\hline Loranthaceae & Elytranthe albida (Blume) Blume & $\mathrm{C} \dagger$ & & & & \\
\hline Loranthaceae & Macrosolen cochinchinensis (Lour.) Tiegh. & $\mathrm{C}$ & & & & \\
\hline Magnoliaceae & Magnolia elegans (Blume) H.Keng & $\mathrm{C}$ & & & & \\
\hline Magnoliaceae & $\begin{array}{l}\text { Magnolia macklottii (Korth.) Dandy var. } \\
\text { beccariana (A.Agostini) Noot. }\end{array}$ & $\mathrm{C} \dagger$ & & & & \\
\hline Magnoliaceae & Magnolia singapurensis (Ridl.) H.Keng & $\mathrm{C}$ & & & & \\
\hline Magnoliaceae & (unidentified) & & 1 & 0 & 0 & \\
\hline Malpighiaceae & Aspidopterys concava (Wall.) A.Juss. & B & 1 & 1 & 1 & \\
\hline Malpighiaceae & Hiptage sericea Hook.f. & & 1 & 0 & 0 & \\
\hline Malvaceae & Brownlowia argentata Kurz & $\mathrm{C} \dagger$ & & & & \\
\hline Malvaceae & Byttneria maingayi Mast. & $\mathrm{C}$ & 1 & 1 & 0 & \\
\hline Malvaceae & Coelostegia griffithii Benth. & $\mathrm{C}$ & & & & \\
\hline Malvaceae & Commersonia bartramia (L.) Merr. & $\mathrm{C}$ & & & & \\
\hline Malvaceae & Durio griffithii (Mast.) Bakh. & $\mathrm{C}$ & 1 & 1 & 0 & \\
\hline Malvaceae & Durio singaporensis Ridl. & $\mathrm{C}$ & 1 & 0 & 0 & \\
\hline Malvaceae & Durio zibethinus L. & & 0 & 1 & 1 & $\mathrm{NN}$ \\
\hline Malvaceae & Grewia laevigata Vahl & & 0 & 1 & 0 & \\
\hline Malvaceae & Heritiera borneensis (Merr.) Kosterm. & $\mathrm{C}$ & 1 & 0 & 0 & \\
\hline
\end{tabular}


Appendix I. Continuation.

\begin{tabular}{|c|c|c|c|c|c|c|}
\hline Family & Accepted Name & Ref. & $\mathbf{P}$ & $\mathbf{O}$ & $\mathbf{M}$ & $\mathbf{N N}$ \\
\hline Malvaceae & Heritiera elata Ridl. & $\mathrm{C}$ & 1 & 1 & 0 & \\
\hline Malvaceae & Heritiera simplicifolia (Mast.) Kosterm. & $\mathrm{C}$ & 0 & 1 & 0 & \\
\hline Malvaceae & Microcos globulifera (Mast.) Burret & $\mathrm{C}^{\dagger}$ & & & & \\
\hline Malvaceae & Microcos hirsuta (Korth.) Burret & $\mathrm{C}$ & & & & \\
\hline Malvaceae & Microcos latifolia Burret & $\mathrm{C}$ & 1 & 0 & 0 & \\
\hline Malvaceae & Neesia synandra Mast. & $\mathrm{C}$ & & & & \\
\hline Malvaceae & Pentace triptera Mast. & $\mathrm{C}$ & 1 & 1 & 0 & \\
\hline Malvaceae & Pterocymbium tubulatum (Mast.) Pierre & $\mathrm{C}$ & & & & \\
\hline Malvaceae & Pterospermum javanicum Jungh. & $\mathrm{C}$ & 1 & 1 & 0 & \\
\hline Malvaceae & $\begin{array}{l}\text { Scaphium macropodum (Miq.) Beumée ex } \\
\text { K.Heyne }\end{array}$ & $\mathrm{C}$ & 1 & 1 & 0 & \\
\hline Malvaceae & Sterculia cordata Blume & B & 0 & 1 & 0 & \\
\hline Malvaceae & $\begin{array}{l}\text { Sterculia lanceolata Blume var. coccinea (Jack) } \\
\text { Phengklai }\end{array}$ & $\mathrm{C}$ & 1 & 1 & 1 & \\
\hline Malvaceae & Sterculia parviflora Roxb. & & 1 & 1 & 0 & \\
\hline Malvaceae & Sterculia rubiginosa Vent. & $\mathrm{C}$ & 1 & 1 & 1 & \\
\hline Marantaceae & Donax canniformis (G.Forst.) K.Schum. & $\mathrm{C}$ & 1 & 0 & 0 & \\
\hline Marantaceae & Phrynium villosulum Miq. & $\mathrm{C}$ & & & & $\mathrm{NN}$ \\
\hline Marantaceae & Stachyphrynium latifolium (Blume) K.Schum. & $\mathrm{C}$ & 1 & 0 & 0 & \\
\hline Marantaceae & $\begin{array}{l}\text { Thaumatococcus daniellii (Benn.) Benth. ex } \\
\text { Eichler }\end{array}$ & A & & & & \\
\hline Melastomataceae & Clidemia hirta (L.) D.Don & $\mathrm{C}$ & 1 & 1 & 1 & NN \\
\hline Melastomataceae & Diplectria divaricata (Willd.) Kuntze & B & 1 & 0 & 0 & \\
\hline Melastomataceae & Dissochaeta annulata Hook.f. ex Triana & $\mathrm{C} \dagger$ & & & & \\
\hline Melastomataceae & Dissochaeta biligulata Korth. & $\mathrm{C}$ & & & & \\
\hline
\end{tabular}


Appendix I. Continuation.

\begin{tabular}{|c|c|c|c|c|c|c|}
\hline Family & Accepted Name & Ref. & $\mathbf{P}$ & $\mathbf{O}$ & $\mathbf{M}$ & $\mathbf{N N}$ \\
\hline Melastomataceae & Dissochaeta gracilis (Jack) Blume & $\mathrm{C}$ & 0 & 1 & 0 & \\
\hline Melastomataceae & Dissochaeta pallida (Jack) Blume & $\mathrm{C}$ & & & & \\
\hline Melastomataceae & Dissochaeta punctulata Hook.f. ex Triana & $\mathrm{C}$ & & & & \\
\hline Melastomataceae & Lijndenia laurina Zoll. \& Moritzi & $\mathrm{C}$ & 1 & 0 & 0 & \\
\hline Melastomataceae & Macrolenes echinulata (Naudin) Bakh.f. & $\mathrm{C}$ & & & & \\
\hline Melastomataceae & Melastoma malabathricum $\mathrm{L}$. & $\mathrm{C}$ & 1 & 1 & 0 & \\
\hline Melastomataceae & Memecylon amplexicaule Roxb. & $\mathrm{C}$ & 1 & 1 & 0 & \\
\hline Melastomataceae & Memecylon campanulatum C.B.Clarke & $\mathrm{C}$ & 1 & 1 & 0 & \\
\hline Melastomataceae & Memecylon cantleyi Ridl. & & 1 & 0 & 0 & \\
\hline Melastomataceae & Memecylon acuminatum Sm. var. acuminatum & $\mathrm{B}$ & 1 & 1 & 0 & \\
\hline Melastomataceae & Memecylon durum Cogn. & $\mathrm{C} \dagger$ & & & & \\
\hline Melastomataceae & Memecylon edule Roxb. & $\mathrm{C}$ & & & & \\
\hline Melastomataceae & Memecylon excelsum Blume & & 1 & 1 & 0 & \\
\hline Melastomataceae & Memecylon floridum Ridl. & $\mathrm{C}$ & 1 & 1 & 0 & \\
\hline Melastomataceae & Memecylon garcinioides Blume & $\mathrm{C}$ & & & & \\
\hline Melastomataceae & Memecylon megacarpum Furtado & $\mathrm{C}$ & 1 & 0 & 0 & \\
\hline Melastomataceae & Memecylon minutiflorum Miq. & $\mathrm{C}$ & 1 & 1 & 0 & \\
\hline Melastomataceae & Memecylon paniculatum Jack & & 1 & 0 & 0 & \\
\hline Melastomataceae & Memecylon pubescens (C.B.Clarke) King & $\mathrm{C}$ & 0 & 1 & 0 & \\
\hline Melastomataceae & Ochthocharis javanica Blume & $\mathrm{C} \dagger$ & & & & \\
\hline Melastomataceae & $\begin{array}{l}\text { Pachycentria glauca Triana subsp. maingayi } \\
\text { (C.B.Clarke) Clausing }\end{array}$ & $\mathrm{C}$ & & & & \\
\hline Melastomataceae & Pachycentria pulverulenta (Jack) Clausing & $\mathrm{C}$ & & & & \\
\hline Melastomataceae & Pternandra coerulescens Jack & $\mathrm{C}$ & 1 & 1 & 0 & \\
\hline
\end{tabular}


Appendix I. Continuation.

\begin{tabular}{|c|c|c|c|c|c|c|}
\hline Family & Accepted Name & Ref. & $\mathbf{P}$ & $\mathbf{O}$ & M & $\mathbf{N N}$ \\
\hline Melastomataceae & Pternandra echinata Jack & $\mathrm{C}$ & 1 & 1 & 0 & \\
\hline Melastomataceae & Pternandra tuberculata (Korth.) M.P.Nayar & $\mathrm{C}$ & & & & \\
\hline Melastomataceae & Sonerila moluccana Roxb. & $\mathrm{C}$ & & & & \\
\hline Melastomataceae & Sonerila obliqua Korth. & $\mathrm{C}$ & & & & \\
\hline Meliaceae & Aglaia crassinervia Kurz ex Hiern & A & 1 & 1 & 0 & \\
\hline Meliaceae & Aglaia exstipulata (Griff.) W.Theob. & $\mathrm{C}$ & & & & \\
\hline Meliaceae & Aglaia leptantha Miq. & $\mathrm{C}$ & 0 & 1 & 0 & \\
\hline Meliaceae & Aglaia malaccensis (Ridl.) Pannell & $\mathrm{C}$ & 1 & 0 & 0 & \\
\hline Meliaceae & Aglaia meliosmoides Craib & $\mathrm{C}$ & & & & \\
\hline Meliaceae & Aglaia odoratissima Blume & $\mathrm{C}$ & 1 & 0 & 0 & \\
\hline Meliaceae & Aglaia oligophylla Miq. & $\mathrm{C} \dagger$ & & & & \\
\hline Meliaceae & Aglaia palembanica Miq. & B & 0 & 1 & 0 & \\
\hline Meliaceae & Aglaia rubiginosa (Hiern) Pannell & $\mathrm{C}$ & & & & \\
\hline Meliaceae & Aglaia rufinervis (Blume) Bentv. & $\mathrm{C}$ & 1 & 0 & 0 & \\
\hline Meliaceae & Aglaia teysmanniana (Miq.) Miq. & & 1 & 0 & 0 & \\
\hline Meliaceae & Aphanamixis polystachya (Wall.) Parker & $\mathrm{C}$ & & & & \\
\hline Meliaceae & Chisocheton patens Blume & $\mathrm{C}$ & 1 & 1 & 0 & \\
\hline Meliaceae & $\begin{array}{l}\text { Chisocheton pentandrus (Blanco) Merr. subsp. } \\
\text { paucijugus (Miq.) Mabb. }\end{array}$ & $\mathrm{B}$ & & & & \\
\hline Meliaceae & Chisocheton sarawakanus (C.DC.) Harms & & 0 & 1 & 0 & \\
\hline Meliaceae & Dysoxylum acutangulum Miq. & $\mathrm{C}$ & & & & \\
\hline Meliaceae & Dysoxylum alliaceum (Blume) Blume & $\mathrm{C} \dagger$ & & & & \\
\hline Meliaceae & Dysoxylum cauliflorum Hiern & $\mathrm{C}$ & 1 & 1 & 1 & \\
\hline Meliaceae & Dysoxylum cyrtobotryum Miq. & $\mathrm{C}^{\dagger}$ & & & & \\
\hline
\end{tabular}


Appendix I. Continuation.

\begin{tabular}{|c|c|c|c|c|c|c|}
\hline Family & Accepted Name & Ref. & $\mathbf{P}$ & $\mathbf{O}$ & $\mathbf{M}$ & $\mathbf{N N}$ \\
\hline Meliaceae & Dysoxylum excelsum Blume & $\mathrm{B}$ & & & & \\
\hline Meliaceae & Dysoxylum grande Hiern & A & & & & \\
\hline Meliaceae & Dysoxylum sp. & & 0 & 1 & 0 & \\
\hline Meliaceae & Lansium domesticum Corrêa & $\mathrm{C}$ & 0 & 1 & 1 & $\mathrm{NN}$ \\
\hline Meliaceae & Pseudoclausena chrysogyne (Miq.) T.P.Clark & $\mathrm{C}$ & 1 & 1 & 0 & \\
\hline Meliaceae & Sandoricum beccarianum Baill. & $\mathrm{C}$ & 1 & 1 & 0 & \\
\hline Meliaceae & Sandoricum koetjape (Burm.f.) Merr. & $\mathrm{C}$ & 1 & 1 & 0 & \\
\hline Menispermaceae & Coscinium fenestratum (Gaertn.) Colebr. & $\mathrm{C}$ & 0 & 1 & 0 & \\
\hline Menispermaceae & Cyclea laxiflora Miers & $\mathrm{C}$ & & & & \\
\hline Menispermaceae & Fibraurea tinctoria Lour. & $\mathrm{C}$ & 1 & 1 & 1 & \\
\hline Menispermaceae & Limacia scandens Lour. & $\mathrm{C}$ & 1 & 1 & 0 & \\
\hline Menispermaceae & $\begin{array}{l}\text { Tinomiscium petiolare Miers ex Hook.f. \& } \\
\text { Thomson }\end{array}$ & $\mathrm{C}$ & 1 & 1 & 1 & \\
\hline Menispermaceae & Tinospora macrocarpa Diels & & 0 & 1 & 0 & \\
\hline Monimiaceae & Kibara coriacea (Blume) Hook.f. \& Thomson & $\mathrm{C}$ & & & & \\
\hline Monimiaceae & Matthaea sancta Blume & $\mathrm{C}$ & 1 & 0 & 0 & \\
\hline Moraceae & Antiaris toxicaria Lesch. & B & 1 & 1 & 0 & \\
\hline Moraceae & Artocarpus anisophyllus Miq. & & 1 & 0 & 0 & \\
\hline Moraceae & Artocarpus elasticus Reinw. ex Blume & $\mathrm{C}$ & 1 & 1 & 1 & \\
\hline Moraceae & Artocarpus heterophyllus Lam. & & 0 & 1 & 0 & $\mathrm{NN}$ \\
\hline Moraceae & Artocarpus hispidus F.M.Jarrett & $\mathrm{C}$ & 1 & 1 & 0 & \\
\hline Moraceae & Artocarpus integer (Thunb.) Merr. & $\mathrm{C}$ & 0 & 0 & 1 & $\mathrm{NN}$ \\
\hline Moraceae & Artocarpus kemando Miq. & $\mathrm{C}$ & & & & \\
\hline Moraceae & Artocarpus lacucha Buch.-Ham. & $\mathrm{C}$ & 1 & 0 & 0 & \\
\hline
\end{tabular}


Appendix I. Continuation.

\begin{tabular}{|c|c|c|c|c|c|c|}
\hline Family & Accepted Name & Ref. & $\mathbf{P}$ & $\mathbf{O}$ & $\mathbf{M}$ & $\mathbf{N N}$ \\
\hline Moraceae & Artocarpus lanceifolius Roxb. & $\mathrm{C}$ & 1 & 1 & 0 & \\
\hline Moraceae & Artocarpus lowii King & $\mathrm{C}$ & 1 & 0 & 0 & \\
\hline Moraceae & $\begin{array}{l}\text { Artocarpus nitidus Trécul subsp. griffithii } \\
\text { (King) F.M.Jarrett }\end{array}$ & & 0 & 1 & 1 & \\
\hline Moraceae & Artocarpus rigidus Blume & $\mathrm{C}$ & 1 & 0 & 0 & \\
\hline Moraceae & Ficus annulata Blume & $\mathrm{C}^{\dagger}$ & & & & \\
\hline Moraceae & Ficus apiocarpa Miq. & & 0 & 1 & 0 & \\
\hline Moraceae & Ficus aurata Miq. var. aurata & $\mathrm{C}$ & 1 & 1 & 0 & \\
\hline Moraceae & Ficus chartacea Wall. ex King & $\mathrm{C}$ & 1 & 1 & 0 & \\
\hline Moraceae & Ficus consociata Blume var. murtonii King & $\mathrm{C}$ & & & & \\
\hline Moraceae & Ficus crassiramea Miq. & $\mathrm{C}$ & & & & \\
\hline Moraceae & Ficus deltoidea Jack & $\mathrm{C}$ & & & & \\
\hline Moraceae & Ficus dubia Wall. ex King & $\mathrm{C}$ & & & & \\
\hline Moraceae & Ficus fistulosa Reinw. ex Blume & $\mathrm{C}$ & 1 & 1 & 1 & \\
\hline Moraceae & Ficus glandulifera (Wall. ex Miq.) King & $\mathrm{C}$ & 1 & 0 & 0 & \\
\hline Moraceae & Ficus globosa Blume & $\mathrm{C}$ & 1 & 0 & 0 & \\
\hline Moraceae & $\begin{array}{l}\text { Ficus grossularioides Burm.f. var. } \\
\text { grossularioides }\end{array}$ & $\mathrm{C}$ & 1 & 1 & 1 & \\
\hline Moraceae & Ficus heteropleura Blume & B & 1 & 1 & 0 & \\
\hline Moraceae & Ficus kerkhovenii Valeton & $\mathrm{C}$ & & & & \\
\hline Moraceae & Ficus laevis Blume & $\mathrm{C}$ & 0 & 1 & 0 & \\
\hline Moraceae & Ficus lamponga Miq. & $\mathrm{C}$ & & & & \\
\hline Moraceae & Ficus microcarpa L.f. & $\mathrm{C}$ & & & & \\
\hline Moraceae & Ficus pallescens (Weiblen) C.C.Berg & $\mathrm{C}$ & 1 & 0 & 0 & \\
\hline Moraceae & Ficus pumila $\mathrm{L}$. & & 1 & 0 & 0 & NN \\
\hline
\end{tabular}


Appendix I. Continuation.

\begin{tabular}{|c|c|c|c|c|c|c|}
\hline Family & Accepted Name & Ref. & $\mathbf{P}$ & $\mathbf{O}$ & $\mathbf{M}$ & NN \\
\hline Moraceae & Ficus punctata Thunb. & $\mathrm{C}$ & 1 & 1 & 1 & $\mathrm{NN}$ \\
\hline Moraceae & Ficus recurva Blume var. ribesoides King & $\mathrm{C}$ & & & & \\
\hline Moraceae & Ficus rosulata C.C.Berg & $\mathrm{C}$ & 0 & 1 & 0 & \\
\hline Moraceae & Ficus ruginervia Corner & $\mathrm{C}$ & 1 & 0 & 0 & \\
\hline Moraceae & Ficus sagittata Vahl & $\mathrm{C}$ & 1 & 1 & 0 & \\
\hline Moraceae & Ficus scortechinii King & $\mathrm{C}$ & & & & \\
\hline Moraceae & Ficus sinuata Thunb. & $\mathrm{C}$ & & & & \\
\hline Moraceae & Ficus subgelderi Corner & $\mathrm{C}^{\dagger}$ & & & & \\
\hline Moraceae & Ficus sundaica Blume var. sundaica & $\mathrm{C}$ & & & & \\
\hline Moraceae & Ficus variegata Blume & $\mathrm{C}$ & 1 & 0 & 1 & \\
\hline Moraceae & Ficus vasculosa Wall. ex Miq. & $\mathrm{C}$ & 0 & 1 & 0 & \\
\hline Moraceae & Ficus villosa Blume & $\mathrm{C}$ & 1 & 1 & 0 & \\
\hline Moraceae & Ficus virens Aiton & $\mathrm{C}$ & & & & \\
\hline Moraceae & Ficus xylophylla Wall. ex Miq. & $\mathrm{C}$ & & & & \\
\hline Moraceae & Streblus elongatus (Miq.) Corner & $\mathrm{C}$ & 1 & 1 & 0 & \\
\hline Muntingiaceae & Muntingia calabura 1 . & A & & & & \\
\hline Musaceae & Musa sp. & & 0 & 1 & 0 & \\
\hline Myristicaceae & Endocomia canarioides (King) W.J.de Wilde & $\mathrm{C}$ & & & & \\
\hline Myristicaceae & $\begin{array}{l}\text { Gymnacranthera farquhariana (Wall. ex } \\
\text { Hook.f. \& Thomson) Warb. var. eugeniifolia } \\
\text { (A.DC.) R.T.A.Schouten }\end{array}$ & $\mathrm{C}$ & 1 & 1 & 0 & \\
\hline Myristicaceae & Gymnacranthera forbesii (King) Warb. & $\mathrm{C}$ & 1 & 0 & 0 & \\
\hline Myristicaceae & $\begin{array}{l}\text { Horsfieldia crassifolia (Hook.f. \& Thomson) } \\
\text { Warb. }\end{array}$ & $\mathrm{C}$ & & & & \\
\hline Myristicaceae & Horsfieldia grandis (Hook.f.) Warb. & & 1 & 0 & 0 & \\
\hline
\end{tabular}


Appendix I. Continuation.

\begin{tabular}{|c|c|c|c|c|c|c|}
\hline Family & Accepted Name & Ref. & $\mathbf{P}$ & $\mathbf{O}$ & $\mathbf{M}$ & $\mathbf{N N}$ \\
\hline Myristicaceae & Horsfieldia polyspherula (Hook.f.) J.Sinclair & $\mathrm{C}$ & 1 & 1 & 0 & \\
\hline Myristicaceae & Horsfieldia punctatifolia J.Sinclair & $\mathrm{C}$ & & & & \\
\hline Myristicaceae & $\begin{array}{l}\text { Horsfieldia superba (Hook.f. \& Thomson) } \\
\text { Warb. }\end{array}$ & $\mathrm{C}$ & 1 & 0 & 0 & \\
\hline Myristicaceae & $\begin{array}{l}\text { Horsfieldia wallichii (Hook.f. \& Thomson) } \\
\text { Warb. }\end{array}$ & $\mathrm{C}$ & & & & \\
\hline Myristicaceae & Knema communis J.Sinclair & $\mathrm{C}$ & 1 & 1 & 0 & \\
\hline Myristicaceae & Knema conferta (King) Warb. & $\mathrm{C}$ & 0 & 1 & 0 & \\
\hline Myristicaceae & $\begin{array}{l}\text { Knema hookeriana (Wall. ex Hook.f. \& } \\
\text { Thomson) Warb. }\end{array}$ & $\mathrm{C}$ & 1 & 0 & 0 & \\
\hline Myristicaceae & Knema intermedia (Blume) Warb. & $\mathrm{C}$ & 1 & 1 & 0 & \\
\hline Myristicaceae & $\begin{array}{l}\text { Knema latericia Elmer subsp. ridleyi (Gand.) } \\
\text { W.J.de Wilde }\end{array}$ & $\mathrm{C}$ & 1 & 1 & 0 & \\
\hline Myristicaceae & Knema laurina (Blume) Warb. & $\mathrm{C}$ & 1 & 1 & 0 & \\
\hline Myristicaceae & Knema malayana Warb. & & 1 & 1 & 0 & \\
\hline Myristicaceae & Knema rubens (J.Sinclair) W.J.de Wilde & $\mathrm{C}$ & & & & \\
\hline Myristicaceae & Knema cf. sumatrana (Blume) W.J.de Wilde & & 1 & 0 & 0 & \\
\hline Myristicaceae & Myristica cinnamomea King & $\mathrm{C}$ & & & & \\
\hline Myristicaceae & Myristica crassa King & & 0 & 1 & 0 & \\
\hline Myristicaceae & $\begin{array}{l}\text { Myristica elliptica Wall. ex Hook.f. \& } \\
\text { Thomson }\end{array}$ & $\mathrm{C}$ & 1 & 0 & 0 & \\
\hline Myristicaceae & Myristica iners Blume & & 1 & 0 & 0 & \\
\hline Myristicaceae & Myristica lowiana King & $\mathrm{C}$ & & & & \\
\hline Myristicaceae & Myristica maingayi Hook.f. & $\mathrm{C}$ & 1 & 0 & 0 & \\
\hline Myristicaceae & Myristica maxima Warb. & $\mathrm{C}$ & & & & \\
\hline Myristicaceae & Myristica sp. & & 1 & 0 & 0 & \\
\hline
\end{tabular}


Appendix I. Continuation.

\begin{tabular}{|c|c|c|c|c|c|c|}
\hline Family & Accepted Name & Ref. & $\mathbf{P}$ & $\mathbf{O}$ & $\mathbf{M}$ & $\mathbf{N N}$ \\
\hline Myrtaceae & Decaspermum parviflorum (Lam.) A.J.Scott & $\mathrm{C}$ & & & & \\
\hline Myrtaceae & Rhodamnia cinerea Jack & $\mathrm{C}$ & 1 & 1 & 0 & \\
\hline Myrtaceae & Rhodomyrtus tomentosa (Aiton) Hassk. & $\mathrm{C}$ & & & & \\
\hline Myrtaceae & Syzygium acuminatissimum (Blume) DC. & & 0 & 1 & 0 & \\
\hline Myrtaceae & $\begin{array}{l}\text { Syzygium attenuatum (Miq.) Merr. \& } \\
\text { L.M.Perry var. attenuatum }\end{array}$ & $\mathrm{C}$ & 1 & 0 & 1 & \\
\hline Myrtaceae & Syzygium borneense (Miq.) Miq. & $\mathrm{C}$ & 1 & 1 & 1 & \\
\hline Myrtaceae & $\begin{array}{l}\text { Syzygium chloranthum (Duthie) Merr. \& } \\
\text { L.M.Perry }\end{array}$ & $\mathrm{C}$ & 1 & 0 & 0 & \\
\hline Myrtaceae & $\begin{array}{l}\text { Syzygium claviflorum (Roxb.) Wall. ex } \\
\text { A.M.Cowan \& Cowan var. claviflorum }\end{array}$ & & 1 & 1 & 0 & \\
\hline Myrtaceae & Syzygium duthieanum (King) Masam. & $\mathrm{C}$ & 1 & 1 & 0 & \\
\hline Myrtaceae & $\begin{array}{l}\text { Syzygium filiforme (Wall. ex Duthie) Chantar. } \\
\text { \& J.Parn. var. filiforme }\end{array}$ & $\mathrm{C}$ & 1 & 1 & 0 & \\
\hline Myrtaceae & Syzygium cf. glabratum (DC.) Veldkamp & & 1 & 0 & 0 & \\
\hline Myrtaceae & Syzygium glaucum (King) Chantar. \& J.Parn. & $\mathrm{C}$ & 1 & 1 & 0 & \\
\hline Myrtaceae & Syzygium grande (Wight) Walp. & $\mathrm{C}$ & 0 & 1 & 0 & \\
\hline Myrtaceae & $\begin{array}{l}\text { Syzygium incarnatum (Elmer) Merr. \& } \\
\text { L.M.Perry }\end{array}$ & $\mathrm{C}$ & 1 & 0 & 0 & \\
\hline Myrtaceae & Syzygium inophyllum DC. & $\mathrm{C}$ & & & & \\
\hline Myrtaceae & $\begin{array}{l}\text { Syzygium leptostemon (Korth.) Merr. \& } \\
\text { L.M.Perry }\end{array}$ & $\mathrm{C}^{\dagger}$ & & & & \\
\hline Myrtaceae & Syzygium lineatum (DC.) Merr. \& L.M.Perry & $\mathrm{C}$ & 0 & 1 & 1 & \\
\hline Myrtaceae & Syzygium linocieroideum (King) I.M.Turner & $\mathrm{C}$ & 1 & 0 & 0 & \\
\hline Myrtaceae & $\begin{array}{l}\text { Syzygium ngadimanianum (M.R.Hend.) } \\
\text { I.M.Turner }\end{array}$ & $\mathrm{C}$ & & & & \\
\hline Myrtaceae & Syzygium nigricans (King) Merr. \& L.M.Perry & $\mathrm{C}$ & 1 & 1 & 0 & \\
\hline
\end{tabular}


Appendix I. Continuation.

\begin{tabular}{|c|c|c|c|c|c|c|}
\hline Family & Accepted Name & Ref. & $\mathbf{P}$ & $\mathbf{O}$ & $\mathbf{M}$ & $\mathbf{N N}$ \\
\hline Myrtaceae & $\begin{array}{l}\text { Syzygium pachyphyllum (Kurz) Merr. \& } \\
\text { L.M.Perry }\end{array}$ & $\mathrm{C}$ & 0 & 1 & 0 & \\
\hline Myrtaceae & Syzygium palembanicum Miq. & $\mathrm{C}$ & & & & \\
\hline Myrtaceae & $\begin{array}{l}\text { Syzygium papillosum (Duthie) Merr. \& } \\
\text { L.M.Perry }\end{array}$ & $\mathrm{C}$ & & & & \\
\hline Myrtaceae & Syzygium pauper (Ridl.) I.M.Turner & $\mathrm{C}$ & & & & \\
\hline Myrtaceae & Syzygium pendens (Duthie) I.M.Turner & $\mathrm{C}$ & 1 & 1 & 0 & \\
\hline Myrtaceae & Syzygium polyanthum (Wight) Walp. & $\mathrm{C}$ & 0 & 0 & 1 & \\
\hline Myrtaceae & $\begin{array}{l}\text { Syzygium pseudoformosum (King) Merr. \& } \\
\text { L.M.Perry }\end{array}$ & $\mathrm{C}$ & 1 & 1 & 0 & \\
\hline Myrtaceae & Syzygium pustulatum (Duthie) Merr. & & 1 & 1 & 0 & \\
\hline Myrtaceae & Syzygium pycnanthum Merr. \& L.M.Perry & $\mathrm{C}$ & 1 & 1 & 0 & \\
\hline Myrtaceae & Syzygium pyrifolium (Blume) DC. & $\mathrm{C}$ & 0 & 1 & 0 & \\
\hline Myrtaceae & Syzygium ridleyi (King) Chantar. \& J.Parn. & $\mathrm{C}$ & 1 & 0 & 0 & \\
\hline Myrtaceae & Syzygium rugosum Korth. var. rugosum & $\mathrm{C}$ & 1 & 1 & 0 & \\
\hline Myrtaceae & Syzygium singaporense (King) Airy Shaw & & 1 & 0 & 0 & \\
\hline Myrtaceae & $\begin{array}{l}\text { Syzygium sp. (Eugenia sp. } 8 \text { sensu } \\
\text { Kochummen) }\end{array}$ & $\mathrm{C}$ & & & & \\
\hline Myrtaceae & $\begin{array}{l}\text { Syzygium subdecussatum (Wall. ex Duthie) } \\
\text { I.M.Turner var. subdecussatum }\end{array}$ & $\mathrm{C}$ & 1 & 1 & 0 & \\
\hline Myrtaceae & $\begin{array}{l}\text { Syzygium syzygioides (Miq.) Merr. \& } \\
\text { L.M.Perry }\end{array}$ & & 1 & 0 & 1 & \\
\hline Myrtaceae & Syzygium urophyllum Merr. & $\mathrm{C} \dagger$ & & & & \\
\hline Myrtaceae & $\begin{array}{l}\text { Tristaniopsis merguensis (Griff.) Peter } \\
\text { G.Wilson \& J.T.Waterh. }\end{array}$ & $\mathrm{C}$ & & & & \\
\hline Nepenthaceae & Nepenthes ampullaria Jack & $\mathrm{C}$ & & & & \\
\hline Nepenthaceae & Nepenthes gracilis Korth. & $\mathrm{C}$ & 0 & 1 & 0 & \\
\hline
\end{tabular}


Appendix I. Continuation.

\begin{tabular}{|c|c|c|c|c|c|c|}
\hline Family & Accepted Name & Ref. & $\mathbf{P}$ & $\mathbf{O}$ & $\mathbf{M}$ & NN \\
\hline Nepenthaceae & Nepenthes $\times$ hookeriana Lindl. & $\mathrm{C}$ & & & & \\
\hline Nymphaeaceae & Barclaya kunstleri (King) Ridl. & $\mathrm{C}$ & & & & \\
\hline Nyssaceae & $\begin{array}{l}\text { Mastixia pentandra Blume subsp. scortechinii } \\
\text { (King) K.M.Matthew }\end{array}$ & $\mathrm{C}$ & 1 & 1 & 0 & \\
\hline Ochnaceae & Brackenridgea elegantissima (Wall.) Kanis & $\mathrm{C}$ & 1 & 0 & 0 & \\
\hline Ochnaceae & $\begin{array}{l}\text { Campylospermum serratum (Gaertn.) Bittrich } \\
\text { \& M.C.E.Amaral }\end{array}$ & $\mathrm{C}$ & 1 & 1 & 0 & \\
\hline Ochnaceae & Euthemis leucocarpa Jack & $\mathrm{C} \dagger$ & & & & \\
\hline Olacaceae & Anacolosa frutescens (Blume) Blume & $\mathrm{C} \uparrow$ & & & & \\
\hline Olacaceae & Erythropalum scandens Blume & & 0 & 1 & 0 & \\
\hline Olacaceae & Ochanostachys amentacea Mast. & $\mathrm{C}$ & 1 & 1 & 0 & \\
\hline Olacaceae & Scorodocarpus borneensis Becc. & $\mathrm{C}$ & 1 & 1 & 0 & \\
\hline Olacaceae & Strombosia ceylanica Gardner & $\mathrm{C}$ & 1 & 1 & 0 & \\
\hline Olacaceae & Strombosia javanica Blume & $\mathrm{C}$ & 1 & 0 & 0 & \\
\hline Oleaceae & Chionanthus ramiflorus Roxb. & $\mathrm{C}$ & & & & \\
\hline Oleaceae & Jasminum elongatum (P.J.Bergius) Willd. & $\mathrm{C}$ & 1 & 0 & 1 & \\
\hline Oleaceae & Olea brachiata (Lour.) Merr. & & 1 & 1 & 0 & \\
\hline Oleaceae & Olea sp. & & 1 & 0 & 0 & \\
\hline Opiliaceae & Cansjera rheedei J.F.Gmel. & $\mathrm{C}$ & 1 & 1 & 0 & \\
\hline Opiliaceae & Champereia manillana (Blume) Merr. & & 0 & 1 & 1 & \\
\hline Orchidaceae & Acriopsis ridleyi Hook.f. & A & & & & \\
\hline Orchidaceae & $\begin{array}{l}\text { Agrostophyllum stipulatum (Griff.) Schltr. } \\
\text { subsp. bicuspidatum (J.J.Sm.) Schuit. }\end{array}$ & $\mathrm{C} \dagger$ & & & & \\
\hline Orchidaceae & Anoectochilus geniculatus Ridl. & $\mathrm{C} \dagger$ & & & & \\
\hline Orchidaceae & Aphyllorchis pallida Blume & $\mathrm{C} \dagger$ & & & & \\
\hline
\end{tabular}


Appendix I. Continuation.

\begin{tabular}{|c|c|c|c|c|c|c|}
\hline Family & Accepted Name & Ref. & $\mathbf{P}$ & $\mathbf{O}$ & $\mathbf{M}$ & $\mathbf{N N}$ \\
\hline Orchidaceae & Apostasia nuda R.Br. & $\mathrm{C}$ & & & & \\
\hline Orchidaceae & Appendicula cornuta Blume & $\mathrm{C} \dagger$ & & & & \\
\hline Orchidaceae & Appendicula lucida Ridl. & $\mathrm{B} \dagger$ & & & & \\
\hline Orchidaceae & Appendicula uncata Ridl. & $\mathrm{B} \dagger$ & & & & \\
\hline Orchidaceae & Bromheadia finlaysoniana (Lindl.) Miq. & $\mathrm{C}$ & 1 & 0 & 0 & \\
\hline Orchidaceae & Bromheadia truncata Seidenf. & $\mathrm{C} \dagger$ & & & & \\
\hline Orchidaceae & Bulbophyllum acuminatum (Ridl.) Ridl. & $\mathrm{C}$ & & & & \\
\hline Orchidaceae & Bulbophyllum macrochilum Rolfe & $\mathrm{C} \dagger$ & & & & \\
\hline Orchidaceae & Bulbophyllum medusae (Lindl.) Rchb.f. & $\mathrm{C} \dagger$ & & & & \\
\hline Orchidaceae & Bulbophyllum patens King ex Hook.f. & $\mathrm{C} \dagger$ & & & & \\
\hline Orchidaceae & Bulbophyllum pileatum Lindl. & $\mathrm{C} \dagger$ & & & & \\
\hline Orchidaceae & Bulbophyllum singaporeanum Schltr. & $\mathrm{C}$ & & & & \\
\hline Orchidaceae & Calanthe pulchra (Blume) Lindl. & $\mathrm{C}$ & & & & \\
\hline Orchidaceae & Claderia viridiflora Hook.f. & $\mathrm{C}$ & & & & \\
\hline Orchidaceae & Cryptostylis arachnites (Blume) Hassk. & $\mathrm{C}^{\dagger}$ & & & & \\
\hline Orchidaceae & Cystorchis variegata Blume & $\mathrm{C} \dagger$ & & & & \\
\hline Orchidaceae & Dendrobium crumenatum Sw. & $\mathrm{C}$ & 1 & 0 & 0 & \\
\hline Orchidaceae & Dendrobium flexile Ridl. & $\mathrm{C}^{\dagger}$ & & & & \\
\hline Orchidaceae & Dendrobium indragiriense Schltr. & $\mathrm{C}$ & & & & \\
\hline Orchidaceae & Dendrobium plicatile Lindl. & $\mathrm{C} \dagger$ & & & & \\
\hline Orchidaceae & Dendrobium subulatum (Blume) Lindl. & $\mathrm{C}$ & & & & \\
\hline Orchidaceae & Didymoplexis pallens Griff. & $\mathrm{C}$ & & & & \\
\hline Orchidaceae & Dienia ophrydis (J.Koenig) Seidenf. & $\mathrm{C}^{\dagger}$ & & & & \\
\hline
\end{tabular}


Appendix I. Continuation.

\begin{tabular}{|c|c|c|c|c|c|c|}
\hline Family & Accepted Name & Ref. & $\mathbf{P}$ & $\mathbf{O}$ & $\mathbf{M}$ & $\mathbf{N N}$ \\
\hline Orchidaceae & Gastrodia javanica (Blume) Lindl. & $\mathrm{C}$ & & & & \\
\hline Orchidaceae & Hetaeria nitida Ridl. & $\mathrm{C} \dagger$ & & & & \\
\hline Orchidaceae & Hetaeria obliqua Blume & $\mathrm{C}$ & & & & \\
\hline Orchidaceae & Hylophila mollis Lindl. & $\mathrm{C}$ & & & & \\
\hline Orchidaceae & Lecanorchis malaccensis Ridl. & $\mathrm{C}$ & & & & \\
\hline Orchidaceae & Mycaranthes obliqua Lindl. & $\mathrm{C} \dagger$ & & & & \\
\hline Orchidaceae & Nervilia punctata (Blume) Makino & $\mathrm{C}^{\dagger}$ & & & & \\
\hline Orchidaceae & Neuwiedia griffithii Rchb.f. & $\mathrm{C}^{\dagger}$ & & & & \\
\hline Orchidaceae & Neuwiedia veratrifolia Blume & $\mathrm{C}$ & & & & \\
\hline Orchidaceae & Oberonia ciliolata Hook.f. & $\mathrm{B} \dagger$ & & & & \\
\hline Orchidaceae & Oberonia dissitiflora Ridl. & $\mathrm{C}^{\dagger}$ & & & & \\
\hline Orchidaceae & Peristylus lacertifer (Lindl.) J.J.Sm. & $\mathrm{B} \dagger$ & & & & \\
\hline Orchidaceae & Pinalia floribunda (Lindl.) Kuntze & A & & & & \\
\hline Orchidaceae & Plocoglottis gigantea (Hook.f.) J.J.Sm. & $\mathrm{C}$ & & & & \\
\hline Orchidaceae & Spathoglottis plicata Blume & $\mathrm{C}$ & & & & \\
\hline Orchidaceae & Stereosandra javanica Blume & $\mathrm{C}^{\dagger}$ & & & & \\
\hline Orchidaceae & Thrixspermum calceolus (Lindl.) Rchb.f. & $\mathrm{B} \dagger$ & & & & \\
\hline Orchidaceae & Thrixspermum ridleyanum Schltr. & $\mathrm{C}^{\dagger}$ & & & & \\
\hline Orchidaceae & Thrixspermum trichoglottis (Hook.f.) Kuntze & $\mathrm{C}$ & & & & \\
\hline Orchidaceae & Trichotosia gracilis (Hook.f.) Kraenzl. & $\mathrm{C}^{\dagger}$ & & & & \\
\hline Orchidaceae & Tropidia curculigoides Lindl. & $\mathrm{C} \dagger$ & & & & \\
\hline Orchidaceae & Vrydagzynea albida (Blume) Blume & $\mathrm{C}$ & & & & \\
\hline Orchidaceae & Vrydagzynea lancifolia Ridl. & $\mathrm{C}$ & & & & \\
\hline
\end{tabular}


Appendix I. Continuation.

\begin{tabular}{|c|c|c|c|c|c|c|}
\hline Family & Accepted Name & Ref. & $\mathbf{P}$ & $\mathbf{O}$ & $\mathbf{M}$ & $\mathbf{N} \mathbf{N}$ \\
\hline Orchidaceae & Zeuxine clandestina Blume & $\mathrm{C}$ & 0 & 0 & 1 & \\
\hline Oxalidaceae & Averrhoa carambola $\mathrm{L}$. & & 0 & 0 & 1 & NN \\
\hline Oxalidaceae & Dapania racemosa Korth. & $\mathrm{C}$ & & & & \\
\hline Oxalidaceae & $\begin{array}{l}\text { Sarcotheca griffithii (Planch. ex Hook.f.) } \\
\text { Hallier f. }\end{array}$ & $\mathrm{C}$ & & & & \\
\hline Pandaceae & Galearia fulva (Tul.) Miq. & $\mathrm{C}$ & 1 & 1 & 0 & \\
\hline Pandaceae & Galearia maingayi Hook.f. & $\mathrm{C}$ & & & & \\
\hline Pandaceae & Microdesmis caseariifolia Planch. ex Hook. & $\mathrm{C}$ & 1 & 0 & 0 & \\
\hline Pandanaceae & Benstonea ornata (Kurz) Callm. \& Buerki & $\mathrm{C}$ & 0 & 1 & 0 & \\
\hline Pandanaceae & Freycinetia angustifolia Blume & $\mathrm{C}$ & 1 & 1 & 0 & \\
\hline Pandanaceae & Freycinetia sumatrana Hemsl. var. sumatrana & & 1 & 1 & 0 & \\
\hline Pandanaceae & Pandanus amaryllifolius Roxb. & & 0 & 0 & 1 & NN \\
\hline Pandanaceae & Pandanus houlletii Carrière & $\mathrm{C}$ & 1 & 1 & 0 & \\
\hline Pandanaceae & Pandanus yvanii Solms & $\mathrm{C}$ & & & & \\
\hline Passifloraceae & $\begin{array}{l}\text { Adenia macrophylla (Blume) Koord. var. } \\
\text { singaporiana (Wall. ex G.Don) W.J.de Wilde }\end{array}$ & $\mathrm{C}$ & & & & \\
\hline Passifloraceae & Passiflora quadriglandulosa Rodschied & A & 1 & 0 & 0 & NN \\
\hline Pentaphragmataceae & $\begin{array}{l}\text { Pentaphragma ellipticum Poulsen var. } \\
\text { ellipticum }\end{array}$ & $\mathrm{C}$ & 1 & 0 & 0 & \\
\hline Pentaphylacaceae & Adinandra acuminata Korth. & $\mathrm{C}$ & 1 & 0 & 0 & \\
\hline Pentaphylacaceae & Adinandra dumosa Jack & $\mathrm{C}$ & 1 & 1 & 0 & \\
\hline Pentaphylacaceae & Adinandra integerrima $\mathrm{T}$.Anderson ex Dyer & $\mathrm{C} \dagger$ & & & & \\
\hline Pentaphylacaceae & Adinandra sp. & & 1 & 0 & 0 & \\
\hline Pentaphylacaceae & Ternstroemia penangiana Choisy & $\mathrm{C}$ & & & & \\
\hline Phyllanthaceae & Actephila excelsa (Dalzell) Müll.Arg. & $\mathrm{C}$ & 1 & 0 & 0 & \\
\hline
\end{tabular}


Appendix I. Continuation.

\begin{tabular}{|c|c|c|c|c|c|c|}
\hline Family & Accepted Name & Ref. & $\mathbf{P}$ & $\mathbf{O}$ & $\mathbf{M}$ & NN \\
\hline Phyllanthaceae & Antidesma coriaceum Tul. & $\mathrm{C}$ & 1 & 1 & 0 & \\
\hline Phyllanthaceae & Antidesma cuspidatum Müll.Arg. & $\mathrm{C}$ & 1 & 1 & 0 & \\
\hline Phyllanthaceae & Antidesma neurocarpum Miq. & $\mathrm{C}$ & 1 & 1 & 0 & \\
\hline Phyllanthaceae & Aporosa benthamiana Hook.f. & $\mathrm{C}$ & 1 & 1 & 0 & \\
\hline Phyllanthaceae & Aporosa confusa Gage & & 1 & 1 & 0 & \\
\hline Phyllanthaceae & Aporosa falcifera Hook.f. & $\mathrm{C}$ & & & & \\
\hline Phyllanthaceae & Aporosa frutescens Blume & $\mathrm{C}$ & 1 & 1 & 1 & \\
\hline Phyllanthaceae & Aporosa lucida (Miq.) Airy Shaw var. lucida & $\mathrm{C}$ & 1 & 1 & 0 & \\
\hline Phyllanthaceae & Aporosa lunata (Miq.) Kurz & $\mathrm{C}$ & & & & \\
\hline Phyllanthaceae & Aporosa microstachya (Tul.) Müll.Arg. & $\mathrm{C}$ & 1 & 1 & 0 & \\
\hline Phyllanthaceae & Aporosa nervosa Hook.f. & $\mathrm{C}$ & 1 & 0 & 0 & \\
\hline Phyllanthaceae & Aporosa nigricans Hook.f. & $\mathrm{C}$ & 1 & 1 & 0 & \\
\hline Phyllanthaceae & Aporosa prainiana King ex Gage & $\mathrm{C}$ & 0 & 1 & 0 & \\
\hline Phyllanthaceae & Aporosa subcaudata Merr. & $\mathrm{C}$ & 1 & 0 & 0 & \\
\hline Phyllanthaceae & Aporosa symplocoides (Hook.f.) Gage & $\mathrm{C}$ & 1 & 1 & 1 & \\
\hline Phyllanthaceae & Baccaurea brevipes Hook.f. & $\mathrm{C}$ & & & & \\
\hline Phyllanthaceae & Baccaurea macrocarpa (Miq.) Müll.Arg. & $\mathrm{C}$ & & & & \\
\hline Phyllanthaceae & Baccaurea maingayi Hook.f. & $\mathrm{C}$ & & & & \\
\hline Phyllanthaceae & Baccaurea minor Hook.f. & B & 0 & 1 & 0 & \\
\hline Phyllanthaceae & Baccaurea motleyana (Müll.Arg.) Müll.Arg. & & 0 & 1 & 1 & \\
\hline Phyllanthaceae & Baccaurea parviflora (Müll.Arg.) Müll.Arg. & $\mathrm{C}$ & 1 & 1 & 0 & \\
\hline Phyllanthaceae & Baccaurea polyneura Hook.f. & $\mathrm{C}$ & 1 & 1 & 0 & \\
\hline Phyllanthaceae & Baccaurea racemosa (Reinw.) Müll.Arg. & $\mathrm{C}$ & 1 & 1 & 0 & \\
\hline
\end{tabular}


Appendix I. Continuation.

\begin{tabular}{|c|c|c|c|c|c|c|}
\hline Family & Accepted Name & Ref. & $\mathbf{P}$ & $\mathbf{O}$ & $\mathbf{M}$ & NN \\
\hline Phyllanthaceae & Baccaurea sumatrana (Miq.) Müll.Arg. & $\mathrm{C}$ & 1 & 1 & 0 & \\
\hline Phyllanthaceae & Breynia discigera Müll.Arg. & B & & & & \\
\hline Phyllanthaceae & Breynia racemosa (Blume) Müll.Arg. & $\mathrm{C}$ & & & & \\
\hline Phyllanthaceae & Bridelia pustulata Hook.f. & $\mathrm{C}$ & & & & \\
\hline Phyllanthaceae & Bridelia stipularis (L.) Blume & $\mathrm{C}$ & & & & \\
\hline Phyllanthaceae & Cleistanthus hirsutulus Hook.f. & $\mathrm{C} \dagger$ & & & & \\
\hline Phyllanthaceae & Cleistanthus macrophyllus Hook.f. & $\mathrm{C}$ & 1 & 0 & 1 & \\
\hline Phyllanthaceae & Cleistanthus sp. / unknown Euphorbiaceae & & 1 & 0 & 0 & \\
\hline Phyllanthaceae & Glochidion borneense (Müll.Arg.) Boerl. & $\mathrm{C}$ & & & & \\
\hline Phyllanthaceae & Glochidion lutescens Blume & $\mathrm{C}$ & & & & \\
\hline Phyllanthaceae & Glochidion singaporense Gage & & 1 & 0 & 0 & \\
\hline Phyllanthaceae & Glochidion superbum Baill. & $\mathrm{C}$ & 0 & 1 & 0 & \\
\hline Phyllanthaceae & $\begin{array}{l}\text { Glochidion zeylanicum (Gaertn.) A.Juss. var. } \\
\text { arborescens (Blume) Chakrab. \& M.Gangop. }\end{array}$ & $\mathrm{C}$ & & & & \\
\hline Phyllanthaceae & $\begin{array}{l}\text { Glochidion zeylanicum (Gaertn.) A.Juss. var. } \\
\text { zeylanicum }\end{array}$ & $\mathrm{C}$ & & & & \\
\hline Phyllanthaceae & Phyllanthus emblica $\mathrm{L}$. & $\mathrm{C}$ & & & & \\
\hline Phyllanthaceae & Phyllanthus reticulatus Poir. & A & & & & \\
\hline Piperaceae & Piper caninum Blume & $\mathrm{C}$ & 1 & 1 & 0 & \\
\hline Piperaceae & Piper flavimarginatum C.DC. & $\mathrm{C}$ & 1 & 1 & 0 & \\
\hline Piperaceae & Piper macropiper Pennant & $\mathrm{C}$ & 1 & 1 & 0 & \\
\hline Piperaceae & Piper muricatum Blume & $\mathrm{C}$ & & & & \\
\hline Piperaceae & Piper neesii (Miq.) P.K.Mukh. & & 1 & 1 & 1 & \\
\hline Piperaceae & Piper ramipilum C.DC. & $\mathrm{C}$ & 1 & 1 & 1 & \\
\hline Piperaceae & Piper ridleyi C.DC. & $\mathrm{C}$ & & & & \\
\hline
\end{tabular}


Appendix I. Continuation.

\begin{tabular}{|c|c|c|c|c|c|c|}
\hline Family & Accepted Name & Ref. & $\mathbf{P}$ & $\mathbf{O}$ & $\mathbf{M}$ & $\mathbf{N N}$ \\
\hline Piperaceae & Piper sarmentosum Roxb. & & 0 & 1 & 1 & \\
\hline Piperaceae & Piper sp. A & & 1 & 0 & 0 & \\
\hline Piperaceae & Piper sp. B & & 0 & 1 & 0 & \\
\hline Plantaginaceae & Adenosma inopinatum Prain & B & & & & \\
\hline Plantaginaceae & Adenosma javanicum (Blume) Koord. & $\mathrm{B}$ & & & & \\
\hline Plantaginaceae & Bacopa monnieri (L.) Pennell & $\mathrm{B}$ & & & & $\mathrm{NN}$ \\
\hline Plantaginaceae & Limnophila villosa Blume & $\mathrm{B}$ & & & & \\
\hline Poaceae & Centotheca lappacea (L.) Desv. & $\mathrm{C}$ & 0 & 1 & 1 & \\
\hline Poaceae & Cyrtococcum accrescens (Trin.) Stapf & B & & & & \\
\hline Poaceae & Echinochloa colona (L.) Link & $\mathrm{B}$ & & & & \\
\hline Poaceae & Eragrostis brownii (Kunth) Nees & B & & & & \\
\hline Poaceae & $\begin{array}{l}\text { Gigantochloa hasskarliana (Kurz) Backer ex } \\
\text { K.Heyne }\end{array}$ & & 0 & 1 & 0 & $\mathrm{NN}$ \\
\hline Poaceae & Gigantochloa ligulata Gamble & $\mathrm{C}$ & & & & \\
\hline Poaceae & Lophatherum gracile Brongn. & $\mathrm{C}$ & 1 & 0 & 0 & \\
\hline Poaceae & Ottochloa nodosa (Kunth) Dandy & & 0 & 0 & 1 & \\
\hline Poaceae & Scrotochloa urceolata (Roxb.) Judz. & $\mathrm{C}$ & 1 & 1 & 0 & \\
\hline Poaceae & Soejatmia ridleyi (Gamble) K.M.Wong & $\mathrm{C}$ & & & & \\
\hline Poaceae & $\begin{array}{l}\text { Thysanolaena latifolia (Roxb. ex Hornem.) } \\
\text { Honda }\end{array}$ & $\mathrm{B}$ & & & & $\mathrm{NN}$ \\
\hline Polygalaceae & Xanthophyllum amoenum Chodat & $\mathrm{C}$ & 0 & 1 & 0 & \\
\hline Polygalaceae & Xanthophyllum discolor Chodat & & 1 & 1 & 0 & \\
\hline Polygalaceae & Xanthophyllum ellipticum Korth. & & 0 & 1 & 0 & \\
\hline Polygalaceae & Xanthophyllum eurhynchum Miq. & $\mathrm{C}$ & 1 & 1 & 0 & \\
\hline Polygalaceae & Xanthophyllum flavescens Roxb. & $\mathrm{C}$ & 1 & 0 & 0 & \\
\hline
\end{tabular}


Appendix I. Continuation.

\begin{tabular}{|c|c|c|c|c|c|c|}
\hline Family & Accepted Name & Ref. & $\mathbf{P}$ & $\mathbf{O}$ & $\mathbf{M}$ & $\mathbf{N N}$ \\
\hline Polygalaceae & $\begin{array}{l}\text { Xanthophyllum griffithii Hook.f. ex A.W.Benn. } \\
\text { subsp. erectum Meijden }\end{array}$ & & 0 & 1 & 0 & \\
\hline Polygalaceae & Xanthophyllum obscurum A.W.Benn. & $\mathrm{C}$ & & & & \\
\hline Polygalaceae & Xanthophyllum stipitatum A.W.Benn. & $\mathrm{C}$ & 1 & 1 & 0 & \\
\hline Polygalaceae & Xanthophyllum vitellinum (Blume) D.Dietr. & $\mathrm{C}$ & 1 & 1 & 0 & \\
\hline Primulaceae & Ardisia purpurea Reinw. ex Blume & $\mathrm{C}$ & 1 & 0 & 0 & \\
\hline Primulaceae & Ardisia ridleyi King \& Gamble & & 1 & 0 & 0 & \\
\hline Primulaceae & Ardisia sanguinolenta Blume & $\mathrm{C}$ & 1 & 1 & 0 & \\
\hline Primulaceae & Ardisia teysmanniana Scheff. & $\mathrm{C}$ & 1 & 1 & 0 & \\
\hline Primulaceae & Ardisia tuberculata Wall. ex A.DC. & $\mathrm{C}$ & & & & \\
\hline Primulaceae & Ardisia sp. A & & 0 & 1 & 0 & \\
\hline Primulaceae & Embelia amentacea C.B.Clarke & $\mathrm{C}$ & 1 & 1 & 0 & \\
\hline Primulaceae & Embelia canescens Jack & $\mathrm{C}$ & & & & \\
\hline Primulaceae & Embelia lampani Scheff. & $\mathrm{C}$ & 1 & 0 & 0 & \\
\hline Primulaceae & Embelia ribes Burm.f. & $\mathrm{C}$ & 0 & 1 & 0 & \\
\hline Primulaceae & Labisia pumila (Blume) Fern.-Vill. & $\mathrm{C}$ & 1 & 0 & 0 & \\
\hline Primulaceae & Maesa ramentacea Wall. ex Roxb. & $\mathrm{C}$ & 1 & 1 & 0 & \\
\hline Putranjavaceae & Drypetes crassipes Pax \& K.Hoffm. & A & & & & \\
\hline Putranjavaceae & Drypetes longifolia (Blume) Pax \& K.Hoffm. & $\mathrm{C}$ & 1 & 0 & 0 & \\
\hline Putranjavaceae & Drypetes pendula Ridl. & $\mathrm{C}$ & 1 & 0 & 0 & \\
\hline Rhamnaceae & Smythea lanceata Summerh. & & 1 & 0 & 0 & \\
\hline Rhamnaceae & Ventilago malaccensis Ridl. & $\mathrm{C}$ & 1 & 1 & 0 & \\
\hline Rhamnaceae & Ventilago sp. A & & 1 & 1 & 1 & \\
\hline Rhamnaceae & Ziziphus calophylla Wall. ex Hook.f. & $\mathrm{C}$ & 1 & 1 & 0 & \\
\hline
\end{tabular}


Appendix I. Continuation.

\begin{tabular}{|c|c|c|c|c|c|c|}
\hline Family & Accepted Name & Ref. & $\mathbf{P}$ & $\mathbf{O}$ & $\mathbf{M}$ & $\mathbf{N N}$ \\
\hline Rhamnaceae & Ziziphus elegans Wall. & $\mathrm{C}$ & 1 & 1 & 0 & \\
\hline Rhamnaceae & Ziziphus horsfieldii Miq. & $\mathrm{C}$ & 1 & 0 & 0 & \\
\hline Rhamnaceae & Ziziphus sp. A & & 0 & 1 & 0 & \\
\hline Rhizophoraceae & Carallia brachiata (Lour.) Merr. & $\mathrm{C}$ & 1 & 1 & 0 & \\
\hline Rhizophoraceae & Gynotroches axillaris Blume & $\mathrm{C}$ & 1 & 1 & 1 & \\
\hline Rhizophoraceae & Pellacalyx axillaris Korth. & $\mathrm{B}$ & 1 & 1 & 1 & \\
\hline Rhizophoraceae & Pellacalyx saccardianus Scort. & $\mathrm{C}$ & 1 & 1 & 1 & \\
\hline Rosaceae & Prunus arborea (Blume) Kalkman & $\mathrm{C}$ & 1 & 0 & 0 & \\
\hline Rosaceae & Prunus polystachya (Hook.f.) Kalkman & $\mathrm{C}$ & 1 & 1 & 1 & \\
\hline Rosaceae & Rubus moluccanus L. var. moluccanus & $\mathrm{B} \dagger$ & & & & \\
\hline Rubiaceae & Aidia auriculata (Wall.) Ridsdale & & 1 & 1 & 0 & \\
\hline Rubiaceae & Aidia densiflora (Wall.) Masam. & $\mathrm{C}$ & 1 & 1 & 0 & \\
\hline Rubiaceae & Canthium malayense K.M.Wong & $\mathrm{C}$ & 0 & 1 & 0 & \\
\hline Rubiaceae & Canthium sp. & & 1 & 0 & 0 & \\
\hline Rubiaceae & Chassalia chartacea Craib & $\mathrm{C}$ & 0 & 1 & 0 & \\
\hline Rubiaceae & Chassalia curviflora (Wall.) Thwaites & $\mathrm{C}$ & 0 & 1 & 0 & \\
\hline Rubiaceae & Chassalia pubescens Ridl. & $\mathrm{C}^{\dagger}$ & & & & \\
\hline Rubiaceae & $\begin{array}{l}\text { Coelospermum truncatum (Wall.) Baill. ex } \\
\text { K.Schum. }\end{array}$ & $\mathrm{C}$ & & & & \\
\hline Rubiaceae & Coptosapelta flavescens Korth. & & 1 & 0 & 0 & \\
\hline Rubiaceae & Coptosapelta griffithii Hook.f. & $\mathrm{C}$ & & & & \\
\hline Rubiaceae & Coptosapelta parviflora Ridl. & $\mathrm{C} \dagger$ & & & & \\
\hline Rubiaceae & Dibridsonia conferta (Korth.) K.M.Wong & $\mathrm{C}$ & 0 & 1 & 0 & \\
\hline Rubiaceae & Diplospora malaccensis Hook.f. & $\mathrm{C}$ & 1 & 1 & 0 & \\
\hline
\end{tabular}


Appendix I. Continuation.

\begin{tabular}{|c|c|c|c|c|c|c|}
\hline Family & Accepted Name & Ref. & $\mathbf{P}$ & $\mathbf{O}$ & $\mathbf{M}$ & NN \\
\hline Rubiaceae & Gaertnera grisea Hook.f. ex C.B.Clarke & $\mathrm{C}$ & 1 & 1 & 0 & \\
\hline Rubiaceae & Gaertnera obesa Hook.f. ex C.B.Clarke & $\mathrm{B}$ & 1 & 0 & 0 & \\
\hline Rubiaceae & Gaertnera viminea Hook.f. ex C.B.Clarke & $\mathrm{C}$ & 1 & 0 & 0 & \\
\hline Rubiaceae & Gardenia elata Ridl. var. elata & $\mathrm{C}$ & & & & \\
\hline Rubiaceae & $\begin{array}{l}\text { Gardenia subcarinata (Corner) Y.W.Low var. } \\
\text { subcarinata }\end{array}$ & & 1 & 1 & 0 & \\
\hline Rubiaceae & Gardeniopsis longifolia Miq. & $\mathrm{B} \dagger$ & & & & \\
\hline Rubiaceae & Geophila pilosa H.Pearson & $\mathrm{C} \uparrow$ & & & & \\
\hline Rubiaceae & Gynochthodes coriacea Blume & $\mathrm{C}$ & 1 & 1 & 1 & \\
\hline Rubiaceae & $\begin{array}{l}\text { Gynochthodes ridleyi (King \& Gamble) } \\
\text { Razafim. \& B.Bremer }\end{array}$ & $\mathrm{C}$ & 1 & 0 & 0 & \\
\hline Rubiaceae & $\begin{array}{l}\text { Gynochthodes rigida (Miq.) Razafim. \& } \\
\text { B.Bremer }\end{array}$ & & 0 & 1 & 0 & \\
\hline Rubiaceae & Hedyotis capitellata Wall. ex G.Don & $\mathrm{C}$ & 0 & 1 & 0 & \\
\hline Rubiaceae & Hedyotis verticillata (L.) Lam. & & 0 & 0 & 1 & \\
\hline Rubiaceae & Hydnophytum formicarum Jack & $\mathrm{C}$ & & & & \\
\hline Rubiaceae & Ixora concinna R.Br. ex Hook.f. & $\mathrm{C} \uparrow$ & & & & \\
\hline Rubiaceae & Ixora congesta Roxb. & $\mathrm{C}$ & 1 & 1 & 0 & \\
\hline Rubiaceae & Ixora lobbii Loudon & $\mathrm{C}$ & & & & \\
\hline Rubiaceae & Ixora pendula Jack var. pendula & $\mathrm{C}$ & 1 & 1 & 0 & \\
\hline Rubiaceae & Ixora umbellata Valeton ex Koord. \& Valeton & $\mathrm{C}$ & & & & \\
\hline Rubiaceae & Jackiopsis ornata (Wall.) Ridsdale & $\mathrm{C}$ & & & & \\
\hline Rubiaceae & Lasianthus appressus Hook.f. & $\mathrm{C}$ & & & & \\
\hline Rubiaceae & Lasianthus attenuatus Jack & $\mathrm{C}$ & 1 & 0 & 0 & \\
\hline Rubiaceae & Lasianthus chryseus Ridl. & $\mathrm{B}$ & & & & \\
\hline
\end{tabular}


Appendix I. Continuation.

\begin{tabular}{|c|c|c|c|c|c|c|}
\hline Family & Accepted Name & Ref. & $\mathbf{P}$ & $\mathbf{O}$ & $\mathbf{M}$ & $\mathbf{N N}$ \\
\hline Rubiaceae & Lasianthus ellipticus Wight & B & 1 & 1 & 0 & \\
\hline Rubiaceae & Lasianthus perakensis King \& Gamble & $\mathrm{C}$ & & & & \\
\hline Rubiaceae & Lasianthus reticulatus Blume & $\mathrm{C}$ & 1 & 1 & 0 & \\
\hline Rubiaceae & Lasianthus ridleyi King \& Gamble & $\mathrm{C}$ & 1 & 0 & 0 & \\
\hline Rubiaceae & Lasianthus cf. stipularis Blume & $\mathrm{C}$ & 1 & 0 & 0 & \\
\hline Rubiaceae & Lasianthus sp. & & 1 & 0 & 0 & \\
\hline Rubiaceae & Mycetia malayana (Wall. ex Ridl.) Craib & $\mathrm{C} \dagger$ & & & & \\
\hline Rubiaceae & Myrmecodia tuberosa Jack & $\mathrm{C} \dagger$ & & & & \\
\hline Rubiaceae & $\begin{array}{l}\text { Nauclea officinalis (Pierre ex Pit.) Merr. \& } \\
\text { Chun }\end{array}$ & $\mathrm{C}$ & 1 & 1 & 0 & \\
\hline Rubiaceae & Oldenlandia prostrata (Blume) Kuntze & $\mathrm{C}$ & 1 & 0 & 0 & \\
\hline Rubiaceae & Ophiorrhiza singaporensis Ridl. & $\mathrm{C}$ & 0 & 1 & 0 & \\
\hline Rubiaceae & Oxyceros bispinosus (Griff.) Tirveng. & $\mathrm{C}$ & 1 & 1 & 1 & \\
\hline Rubiaceae & Oxyceros drupaceus (C.F.Gaertn.) Ridsdale & $\mathrm{C} \dagger$ & & & & \\
\hline Rubiaceae & Oxyceros longiflorus (Lam.) T.Yamaz. & $\mathrm{C}$ & 0 & 1 & 0 & \\
\hline Rubiaceae & $\begin{array}{l}\text { Oxyceros penangianus (King \& Gamble) } \\
\text { Tirveng. }\end{array}$ & $\mathrm{C}$ & & & & \\
\hline Rubiaceae & Paederia verticillata Blume & $\mathrm{C}^{\dagger}$ & & & & \\
\hline Rubiaceae & Pertusadina eurhyncha (Miq.) Ridsdale & $\mathrm{C}$ & 1 & 0 & 0 & \\
\hline Rubiaceae & Porterandia anisophylla (Jack ex Roxb.) Ridl. & $\mathrm{C}$ & 1 & 1 & 0 & \\
\hline Rubiaceae & Prismatomeris glabra (Korth.) Valeton & $\mathrm{C}$ & 1 & 1 & 0 & \\
\hline Rubiaceae & Psychotria cantleyi Ridl. & $\mathrm{C}$ & & & & \\
\hline Rubiaceae & Psychotria deltata I.M.Turner & A & & & & \\
\hline Rubiaceae & Psychotria griffithii Hook.f. & $\mathrm{C}$ & & & & \\
\hline Rubiaceae & Psychotria helferiana Kurz & $\mathrm{C}$ & 0 & 1 & 0 & \\
\hline
\end{tabular}


Appendix I. Continuation.

\begin{tabular}{|c|c|c|c|c|c|c|}
\hline Family & Accepted Name & Ref. & $\mathbf{P}$ & $\mathbf{O}$ & $\mathbf{M}$ & NN \\
\hline Rubiaceae & Psychotria malayana Jack & $\mathrm{C}$ & & & & \\
\hline Rubiaceae & Psychotria ovoidea Wall. ex Hook.f. & $\mathrm{C}$ & 1 & 1 & 1 & \\
\hline Rubiaceae & Psychotria penangensis Hook.f. & $\mathrm{C}$ & 1 & 1 & 0 & \\
\hline Rubiaceae & Psychotria ridleyi King \& Gamble & $\mathrm{C}$ & 1 & 0 & 0 & \\
\hline Rubiaceae & Psychotria rostrata Blume & $\mathrm{C}$ & 1 & 0 & 0 & \\
\hline Rubiaceae & Psychotria sarmentosa Blume & & 1 & 1 & 0 & \\
\hline Rubiaceae & Psychotria megacoma Miq. & $\mathrm{C}$ & & & & \\
\hline Rubiaceae & Psydrax nitida (Craib) K.M.Wong & & 1 & 0 & 0 & \\
\hline Rubiaceae & $\begin{array}{l}\text { Psydrax lucidulus (Miq.) Mahyuni \& } \\
\text { K.M.Wong }\end{array}$ & $\mathrm{C}$ & & & & \\
\hline Rubiaceae & Saprosma glomerulatum King \& Gamble & $\mathrm{C}$ & 1 & 1 & 0 & \\
\hline Rubiaceae & $\begin{array}{l}\text { Schradera membranacea (King) Puff, } \\
\text { R.Buchner \& Greimler }\end{array}$ & $\mathrm{C}$ & & & & \\
\hline Rubiaceae & $\begin{array}{l}\text { Singaporandia macrophylla (R.Br. ex Hook.f.) } \\
\text { K.M.Wong }\end{array}$ & $\mathrm{C}$ & 1 & 1 & 0 & \\
\hline Rubiaceae & Tarenna adpressa (King) Merr. & $\mathrm{C}$ & & & & \\
\hline Rubiaceae & Tarenna costata (Miq.) Merr. & $\mathrm{C}$ & & & & \\
\hline Rubiaceae & Tarenna mollis (Wall. ex Hook.f.) B.L.Rob. & $\mathrm{C}$ & 1 & 0 & 0 & \\
\hline Rubiaceae & Tarenna odorata (Roxb.) B.L.Rob. & $\mathrm{C}$ & & & & \\
\hline Rubiaceae & Timonius flavescens (Jack) Baker & $\mathrm{C}$ & & & & \\
\hline Rubiaceae & Timonius wallichianus (Korth.) Valeton & $\mathrm{C}$ & 1 & 1 & 1 & \\
\hline Rubiaceae & Uncaria attenuata Korth. & $\mathrm{C} \dagger$ & & & & \\
\hline Rubiaceae & Uncaria callophylla Blume ex Korth. & $\mathrm{C} \dagger$ & & & & \\
\hline Rubiaceae & Uncaria cordata (Lour.) Merr. & $\mathrm{C}$ & & & & \\
\hline Rubiaceae & Uncaria gambir (W.Hunter) Roxb. & $\mathrm{C}$ & & & & $\mathrm{NN}$ \\
\hline
\end{tabular}


Appendix I. Continuation.

\begin{tabular}{|c|c|c|c|c|c|c|}
\hline Family & Accepted Name & Ref. & $\mathbf{P}$ & $\mathbf{O}$ & $\mathbf{M}$ & $\mathbf{N N}$ \\
\hline Rubiaceae & $\begin{array}{l}\text { Uncaria lanosa Wall. var. glabrata (Blume) } \\
\text { Ridsdale }\end{array}$ & $\mathrm{C}$ & 0 & 0 & 1 & \\
\hline Rubiaceae & $\begin{array}{l}\text { Uncaria longiflora (Poir.) Merr. var. pteropoda } \\
\text { (Miq.) Ridsdale }\end{array}$ & $\mathrm{C}$ & 1 & 1 & 1 & \\
\hline Rubiaceae & Uncaria cf. roxburghiana Korth. & $\mathrm{C}$ & 1 & 0 & 0 & \\
\hline Rubiaceae & Uncaria sp. & & 0 & 1 & 0 & \\
\hline Rubiaceae & $\begin{array}{l}\text { Urophyllum arboreum (Reinw. ex Blume) } \\
\text { Korth. }\end{array}$ & $\mathrm{C}$ & 1 & 0 & 0 & \\
\hline Rubiaceae & Urophyllum blumeanum (Wight) Hook.f. & $\mathrm{C}$ & 1 & 1 & 0 & \\
\hline Rubiaceae & Urophyllum corymbosum (Blume) Korth. & $\mathrm{C}+$ & & & & \\
\hline Rubiaceae & Urophyllum griffithianum (Wight) Hook.f. & $\mathrm{C}$ & 1 & 1 & 0 & \\
\hline Rubiaceae & Urophyllum hirsutum (Wight) Hook.f. & $\mathrm{C}$ & 1 & 1 & 0 & \\
\hline Rubiaceae & Urophyllum streptopodium Wall. ex Hook.f. & $\mathrm{C}$ & 1 & 1 & 0 & \\
\hline Rutaceae & Clausena excavata Burm.f. & & 0 & 1 & 1 & \\
\hline Rutaceae & $\begin{array}{l}\text { Glycosmis chlorosperma (Blume) Spreng. var. } \\
\text { chlorosperma }\end{array}$ & $\mathrm{C}$ & 1 & 1 & 0 & \\
\hline Rutaceae & Luvunga crassifolia Tanaka & $\mathrm{B}$ & 1 & 1 & 0 & \\
\hline Rutaceae & Maclurodendron porteri (Hook.f.) T.G.Hartley & $\mathrm{C}$ & 1 & 1 & 0 & \\
\hline Rutaceae & Melicope glabra (Blume) T.G.Hartley & $\mathrm{C}$ & 1 & 1 & 0 & \\
\hline Rutaceae & Melicope hookeri T.G.Hartley & $\mathrm{C}$ & 1 & 1 & 0 & \\
\hline Rutaceae & Melicope lunu-ankenda (Gaertn.) T.G.Hartley & $\mathrm{C}$ & 0 & 1 & 0 & \\
\hline Rutaceae & Melicope sp. A & & 0 & 1 & 0 & \\
\hline Rutaceae & Melicope sp. & & 1 & 0 & 0 & \\
\hline Sabiaceae & $\begin{array}{l}\text { Meliosma pinnata (Roxb.) Maxim. subsp. } \\
\text { ridleyi (King) Beusekom }\end{array}$ & $\mathrm{C}$ & 1 & 0 & 0 & \\
\hline Salicaceae & Casearia clarkei King var. clarkei & $\mathrm{C}$ & & & & \\
\hline
\end{tabular}


Appendix I. Continuation.

\begin{tabular}{|c|c|c|c|c|c|c|}
\hline Family & Accepted Name & Ref. & $\mathbf{P}$ & $\mathbf{O}$ & $\mathbf{M}$ & $\mathbf{N N}$ \\
\hline Salicaceae & Casearia lobbiana Turcz. & $\mathrm{C}$ & & & & \\
\hline Salicaceae & Flacourtia rukam Zoll. \& Moritzi & $\mathrm{C}$ & 1 & 0 & 0 & \\
\hline Salicaceae & Homalium grandiflorum Benth. & $\mathrm{C}$ & 0 & 1 & 0 & \\
\hline Salicaceae & Osmelia philippina (Turcz.) Benth. & $\mathrm{C}$ & 1 & 1 & 0 & \\
\hline Santalaceae & Scleropyrum pentandrum (Dennst.) Mabb. & $\mathrm{C}$ & & & & \\
\hline Santalaceae & Viscum articulatum Burm.f. & $\mathrm{C}$ & & & & \\
\hline Sapindaceae & $\begin{array}{l}\text { Dimocarpus longan Lour. var. malesianus } \\
\text { Leenh. }\end{array}$ & & 0 & 0 & 1 & \\
\hline Sapindaceae & Guioa pleuropteris (Blume) Radlk. & $\mathrm{C}$ & 1 & 1 & 0 & \\
\hline Sapindaceae & Guioa pubescens (Zoll. \& Moritzi) Radlk. & $\mathrm{C}$ & 1 & 1 & 0 & \\
\hline Sapindaceae & Lepisanthes fruticosa (Roxb.) Leenh. & A & & & & \\
\hline Sapindaceae & Lepisanthes rubiginosa (Roxb.) Leenh. & & 1 & 0 & 0 & \\
\hline Sapindaceae & Mischocarpus sundaicus Blume & & 1 & 0 & 0 & \\
\hline Sapindaceae & $\begin{array}{l}\text { Nephelium cuspidatum Blume var. eriopetalum } \\
\text { (Miq.) Leenh. }\end{array}$ & B & 1 & 1 & 0 & \\
\hline Sapindaceae & Nephelium lappaceum $\mathrm{L}$. & $\mathrm{C}$ & 1 & 1 & 1 & \\
\hline Sapindaceae & Nephelium laurinum Blume & & 1 & 0 & 0 & \\
\hline Sapindaceae & Nephelium ramboutan-ake (Labill.) Leenh. & $\mathrm{C}$ & 1 & 0 & 0 & \\
\hline Sapindaceae & Pometia pinnata J.R.Forst. \& G.Forst. & $\mathrm{C}$ & 1 & 0 & 0 & \\
\hline Sapindaceae & Trigonachras acuta (Hiern) Radlk. & $\mathrm{C}$ & 1 & 0 & 0 & \\
\hline Sapindaceae & Xerospermum laevigatum Radlk. & $\mathrm{C}$ & 1 & 0 & 0 & \\
\hline Sapindaceae & Xerospermum noronhianum (Blume) Blume & $\mathrm{C}$ & 1 & 1 & 0 & \\
\hline Sapotaceae & Donella lanceolata (Blume) Aubrév. & $\mathrm{C}$ & 1 & 1 & 0 & \\
\hline Sapotaceae & $\begin{array}{l}\text { Madhuca kingiana (Brace ex King \& Gamble) } \\
\text { H.J.Lam }\end{array}$ & $\mathrm{C}$ & 1 & 1 & 0 & \\
\hline
\end{tabular}


Appendix I. Continuation.

\begin{tabular}{|c|c|c|c|c|c|c|}
\hline Family & Accepted Name & Ref. & $\mathbf{P}$ & $\mathbf{O}$ & $\mathbf{M}$ & $\mathbf{N N}$ \\
\hline Sapotaceae & Madhuca malaccensis (C.B.Clarke) H.J.Lam & $\mathrm{C}$ & 1 & 0 & 0 & \\
\hline Sapotaceae & Madhuca sp. & & 0 & 1 & 0 & \\
\hline Sapotaceae & Palaquium gutta (Hook.) Baill. & $\mathrm{C}$ & 1 & 1 & 0 & \\
\hline Sapotaceae & Palaquium hexandrum (Griff.) Baill. & $\mathrm{C}$ & 1 & 0 & 0 & \\
\hline Sapotaceae & Palaquium impressionervium $\mathrm{Ng}$ & A & & & & \\
\hline Sapotaceae & Palaquium microphyllum King \& Gamble & $\mathrm{C}$ & 1 & 1 & 0 & \\
\hline Sapotaceae & Palaquium obovatum (Griff.) Engl. & $\mathrm{C}$ & 1 & 1 & 0 & \\
\hline Sapotaceae & Palaquium oxleyanum Pierre & & 1 & 1 & 0 & \\
\hline Sapotaceae & Palaquium rostratum (Miq.) Burck & $\mathrm{C}$ & & & & \\
\hline Sapotaceae & Payena lucida (G.Don) DC. & $\mathrm{C}$ & 1 & 1 & 0 & \\
\hline Sapotaceae & Payena maingayi C.B.Clarke & $\mathrm{C}$ & & & & \\
\hline Sapotaceae & Payena obscura Burck & $\mathrm{C}$ & 0 & 1 & 0 & \\
\hline Sapotaceae & Planchonella maingayi (C.B.Clarke) P.Royen & $\mathrm{C}$ & 1 & 1 & 0 & \\
\hline Sapotaceae & Planchonella obovata (R.Br.) Pierre & $\mathrm{C}$ & & & & \\
\hline Sapotaceae & Pouteria malaccensis (C.B.Clarke) Baehni & $\mathrm{C}$ & & & & \\
\hline Sapotaceae & Sarcosperma paniculatum (King) Stapf \& King & $\mathrm{C} \dagger$ & & & & \\
\hline Schisandraceae & Kadsura scandens Blume & $\mathrm{C}$ & 1 & 1 & 0 & \\
\hline Simaroubaceae & Brucea javanica (L.) Merr. & & 0 & 1 & 0 & \\
\hline Simaroubaceae & Eurycoma longifolia Jack & $\mathrm{C}$ & 1 & 1 & 0 & \\
\hline Smilacaceae & Smilax calophylla Wall. ex A.DC. & $\mathrm{C}$ & 1 & 1 & 0 & \\
\hline Smilacaceae & Smilax leucophylla Blume & $\mathrm{C}$ & 1 & 1 & 0 & \\
\hline Smilacaceae & Smilax megacarpa A.DC. & $\mathrm{B}$ & 1 & 0 & 0 & \\
\hline Smilacaceae & Smilax myosotiflora A.DC. & & 1 & 0 & 0 & \\
\hline
\end{tabular}


Appendix I. Continuation.

\begin{tabular}{|c|c|c|c|c|c|c|}
\hline Family & Accepted Name & Ref. & $\mathbf{P}$ & $\mathbf{O}$ & $\mathbf{M}$ & NN \\
\hline Smilacaceae & Smilax setosa Miq. & $\mathrm{C}$ & 1 & 1 & 1 & \\
\hline Staphyleaceae & $\begin{array}{l}\text { Dalrympelea sphaerocarpa (Hassk.) Nor- } \\
\text { Ezzaw. }\end{array}$ & $\mathrm{C}$ & & & & \\
\hline Stemonuraceae & Gomphandra quadrifida (Blume) Sleumer & $\mathrm{C}$ & 1 & 1 & 0 & \\
\hline Styracaceae & Styrax benzoin Dryand. var. benzoin & $\mathrm{C}$ & & & & \\
\hline Symplocaceae & Symplocos adenophylla Wall. ex G.Don & & 0 & 1 & 1 & \\
\hline Symplocaceae & Symplocos fasciculata Zoll. & $\mathrm{C}$ & 1 & 0 & 0 & \\
\hline Symplocaceae & $\begin{array}{l}\text { Symplocos odoratissima (Blume) Choisy ex } \\
\text { Zoll. }\end{array}$ & $\mathrm{C} \dagger$ & & & & \\
\hline Symplocaceae & Symplocos rubiginosa Wall. ex DC. & $\mathrm{C}$ & 1 & 1 & 0 & \\
\hline Theaceae & Camellia sp. A & & 0 & 1 & 0 & $\mathrm{NN}$ \\
\hline Theaceae & Gordonia penangensis Ridl. & & 1 & 0 & 0 & \\
\hline Theaceae & Gordonia singaporeana Wall. ex Ridl. & $\mathrm{C}$ & 1 & 1 & 0 & \\
\hline Theaceae & $\begin{array}{l}\text { Polyspora multinervis (King) Orel, Peter } \\
\text { G.Wilson, Curry \& Luu }\end{array}$ & $\mathrm{C}$ & 1 & 0 & 0 & \\
\hline Theaceae & Pyrenaria acuminata Planch. & $\mathrm{C}$ & 1 & 1 & 0 & \\
\hline Thymelaeaceae & Aquilaria malaccensis Lam. & $\mathrm{C}$ & 1 & 1 & 0 & \\
\hline Thymelaeaceae & Enkleia malaccensis Griff. & $\mathrm{C}$ & 1 & 1 & 0 & \\
\hline Thymelaeaceae & Gonystylus confusus Airy Shaw & $\mathrm{C}$ & 1 & 1 & 0 & \\
\hline Thymelaeaceae & Gonystylus maingayi Hook.f. & B & & & & \\
\hline Thymelaeaceae & Linostoma pauciflorum Griff. & $\mathrm{C}$ & 1 & 1 & 0 & \\
\hline Torricelliaceae & $\begin{array}{l}\text { Aralidium pinnatifidum (Jungh. \& de Vriese) } \\
\text { Miq. }\end{array}$ & $\mathrm{C}^{\dagger}$ & & & & \\
\hline Trigoniaceae & Trigoniastrum hypoleucum Miq. & $\mathrm{C}^{\dagger}$ & & & & \\
\hline Triuridaceae & Sciaphila maculata Miers & $\mathrm{C} \dagger$ & & & & \\
\hline Triuridaceae & Sciaphila tenella Blume & $\mathrm{C} \dagger$ & & & & \\
\hline
\end{tabular}


Appendix I. Continuation.

\begin{tabular}{|c|c|c|c|c|c|c|}
\hline Family & Accepted Name & Ref. & $\mathbf{P}$ & $\mathbf{O}$ & $\mathbf{M}$ & $\mathbf{N N}$ \\
\hline Urticaceae & Poikilospermum suaveolens (Blume) Merr. & $\mathrm{C}$ & 1 & 1 & 1 & \\
\hline Urticaceae & Pouzolzia zeylanica (L.) Benn. & $\mathrm{B}$ & & & & \\
\hline Violaceae & Rinorea anguifera (Lour.) Kuntze & $\mathrm{C}$ & 1 & 1 & 0 & \\
\hline Vitaceae & Ampelocissus ascendiflora Latiff & $\mathrm{C}$ & 1 & 0 & 0 & \\
\hline Vitaceae & Ampelocissus cinnamomea (Wall.) Planch. & $\mathrm{B} \uparrow$ & & & & \\
\hline Vitaceae & Ampelocissus elegans Gagnep. & $\mathrm{C}$ & 1 & 1 & 0 & \\
\hline Vitaceae & Ampelocissus gracilis (Wall.) Planch. & $\mathrm{C}$ & 1 & 0 & 0 & \\
\hline Vitaceae & Ampelocissus polystachya (Wall.) Planch. & $\mathrm{C}$ & 1 & 1 & 0 & \\
\hline Vitaceae & Cayratia mollissima (Wall.) Gagnep. & & 1 & 0 & 0 & \\
\hline Vitaceae & Cissus hastata Miq. & $\mathrm{C}$ & 0 & 1 & 0 & \\
\hline Vitaceae & Cissus nodosa Blume & $\mathrm{C}$ & 1 & 0 & 0 & \\
\hline Vitaceae & Cissus repens Lam. & & 1 & 0 & 0 & \\
\hline Vitaceae & Cissus rostrata (Miq.) Planch. & $\mathrm{C}$ & & & & \\
\hline Vitaceae & Leea indica (Burm.f.) Merr. & $\mathrm{C}$ & 0 & 1 & 1 & \\
\hline Vitaceae & Nothocissus spicifera (Griff.) Latiff & $\mathrm{C}$ & 1 & 0 & 0 & \\
\hline Vitaceae & Pterisanthes cissioides Blume & & 0 & 1 & 0 & \\
\hline Vitaceae & Pterisanthes polita (Miq.) M.A.Lawson & $\mathrm{C}$ & 0 & 1 & 0 & \\
\hline Vitaceae & Tetrastigma curtisii (Ridl.) Seuss. & $\mathrm{C}$ & 1 & 1 & 0 & \\
\hline Vitaceae & Tetrastigma dichotomum Planch. & & 1 & 1 & 0 & \\
\hline Vitaceae & Tetrastigma latiffii Veldkamp & $\mathrm{C}$ & 1 & 0 & 0 & \\
\hline Vitaceae & Tetrastigma rafflesiae (Miq.) Planch. & $\mathrm{C}$ & 1 & 0 & 1 & \\
\hline Zingiberaceae & Amomum hastilabium Ridl. & $\mathrm{C} \dagger$ & & & & \\
\hline Zingiberaceae & Amomum xanthophlebium Baker & B & & & & \\
\hline Zingiberaceae & Elettariopsis latiflora Ridl. & $\mathrm{C}$ & 1 & 0 & 0 & \\
\hline
\end{tabular}


Appendix I. Continuation.

\begin{tabular}{|c|c|c|c|c|c|c|}
\hline Family & Accepted Name & Ref. & $\mathbf{P}$ & $\mathbf{O}$ & $\mathbf{M}$ & $\mathbf{N}$ \\
\hline Zingiberaceae & Etlingera maingayi (Baker) R.M.Sm. & $\mathrm{B}$ & & & & \\
\hline Zingiberaceae & $\begin{array}{l}\text { Globba leucantha Miq. var. peninsularis } \\
\text { Holttum }\end{array}$ & $\mathrm{C}$ & 1 & 0 & 0 & \\
\hline Zingiberaceae & Globba variabilis Ridl. subsp. pusilla S.N.Lim & B & 0 & 1 & 0 & \\
\hline Zingiberaceae & Hornstedtia conica Ridl. & & 1 & 0 & 0 & \\
\hline Zingiberaceae & $\begin{array}{l}\text { Hornstedtia scyphifera (J.Koenig) Steud. var. } \\
\text { scyphifera }\end{array}$ & $\mathrm{C}$ & 1 & 1 & 1 & \\
\hline Zingiberaceae & Plagiostachys lateralis (Ridl.) Ridl. & $\mathrm{B} \dagger$ & & & & \\
\hline Zingiberaceae & Plagiostachys mucida Holttum & $\mathrm{C} \dagger$ & & & & \\
\hline Zingiberaceae & Scaphochlamys tenuis Holttum & B & & & & NN \\
\hline Zingiberaceae & Zingiber griffithii Baker & $\mathrm{C}$ & 0 & 1 & 0 & \\
\hline Zingiberaceae & Zingiber puberulum Ridl. var. puberulum & $\mathrm{C}$ & 1 & 0 & 0 & \\
\hline Zingiberaceae & Zingiber singapurense Škorničk. & A & & & & \\
\hline
\end{tabular}


Appendix II. Taxa recorded from BTNR but either omitted from Turner \& Chua (2011) or published after 2011, 120 additional species in all. The species are listed in alphabetical order of the families and then species.

\begin{tabular}{|c|c|c|}
\hline Family & Accepted Name & Reference \\
\hline Achariaceae & Ryparosa scortechinii King & King (1890); Ho et al. (2018) \\
\hline Amaranthaceae & Cyathula prostrata (L.) Blume & Ridley (1900) \\
\hline Anacardiaceae & Gluta malayana (Corner) Ding Hou & Khoo et al. (2018) \\
\hline Anacardiaceae & Mangifera odorata Griff. & Ridley (1900) \\
\hline Anacardiaceae & Mangifera gracilipes Hook.f. & Khoo et al. (2018) \\
\hline Annonaceae & Alphonsea johorensis J.Sinclair & Khoo et al. (2018) \\
\hline Annonaceae & Artabotrys scortechinii King & Chen et al. (2018) \\
\hline Annonaceae & $\begin{array}{l}\text { Dendrokingstonia nervosa (Hook.f. } \\
\& \text { Thomson) Rauschert }\end{array}$ & Lim et al. (2018) \\
\hline Apocynaceae & Genianthus maingayi Hook.f. & Ridley (1900); Keng (1990) \\
\hline Apocynaceae & Hoya obtusifolia Wight & Keng (1990); Rodda \& Lai (2018) \\
\hline Apocynaceae & Willughbeia coriacea Wall. & $\begin{array}{l}\text { Ridley (1900) as Willughbeia firma } \\
\text { Blume }\end{array}$ \\
\hline Araceae & Aglaonema nitidum (Jack) Kunth & Ridley (1900); Keng et al. (1998) \\
\hline Araceae & Cryptocoryne griffithii Schott & Ridley (1900); Keng et al. (1998) \\
\hline Araceae & Epipremnum pinnatum (L.) Engl. & Keng et al. (1998) \\
\hline Araceae & Rhaphidophora maingayi Hook.f. & Keng et al. (1998) \\
\hline Araceae & Scindapsus hederaceus Miq. & Ridley (1900) \\
\hline Araceae & $\begin{array}{l}\text { Scindapsus lucens Bogner \& } \\
\text { P.C.Boyce }\end{array}$ & Ho et al. (2018) \\
\hline Araceae & Scindapsus pictus Hassk. & Ridley (1900); Keng et al. (1998) \\
\hline Arecaceae & $\begin{array}{l}\text { Daemonorops angustifolia (Griff.) } \\
\text { Mart. }\end{array}$ & Keng et al. (1998) \\
\hline Arecaceae & $\begin{array}{l}\text { Daemonorops geniculata (Griff.) } \\
\text { Mart. }\end{array}$ & Ridley (1900) \\
\hline
\end{tabular}


Appendix II. Continuation.

\begin{tabular}{|c|c|c|}
\hline Family & Accepted Name & Reference \\
\hline Arecaceae & Pinanga pectinata Becc. & Keng et al. (1998) \\
\hline Asteraceae & Blumea riparia (Blume) DC. & $\begin{array}{l}\text { Ridley }(1900) \text { as Vernonia scandens } \\
\text { auct. non DC. ["Tombak-Tombak" is } \\
\text { the the Malay name of Blumea riparia]; } \\
\text { Keng (1990) }\end{array}$ \\
\hline Asteraceae & Gynura procumbens (Lour.) Merr. & $\begin{array}{l}\text { Ridley (1900) as Gynura sarmentosa } \\
\text { (Blume) DC.; Keng (1990) }\end{array}$ \\
\hline Bignoniaceae & $\begin{array}{l}\text { Fernandoa adenophylla (G.Don) } \\
\text { Steenis }\end{array}$ & van Steenis (1977) \\
\hline Burseraceae & $\begin{array}{l}\text { Dacryodes nervosa (H.J.Lam) } \\
\text { Leenh. }\end{array}$ & Khoo et al. (2018) \\
\hline Capparaceae & $\begin{array}{l}\text { Capparis micracantha DC. subsp. } \\
\text { korthalsiana (Miq.) M.Jacobs }\end{array}$ & $\begin{array}{l}\text { Keng (1990) as Capparis micracantha } \\
\text { DC }\end{array}$ \\
\hline Celastraceae & $\begin{array}{l}\text { Salacia viminea Wall. ex } \\
\text { M.A.Lawson }\end{array}$ & Keng (1990) \\
\hline Chrysobalanaceae & Parinari oblongifolia Hook.f. & Ridley (1900); Keng (1990) \\
\hline Combretaceae & Terminalia citrina (Gaertn.) Roxb. & Khoo et al. (2018) \\
\hline Commelinaceae & $\begin{array}{l}\text { Amischotolype gracilis (Ridl.) } \\
\text { I.M.Turner }\end{array}$ & $\begin{array}{l}\text { Ridley (1900) as Forrestia mollis } \\
\text { Hassk.; Keng et al. (1998) }\end{array}$ \\
\hline Connaraceae & Rourea fulgens Planch. & Ridley (1900) \\
\hline Convolvulaceae & Erycibe maingayi C.B.Clarke & Keng (1990) \\
\hline Convolvulaceae & $\begin{array}{l}\text { Merremia hederacea (Burm.f.) } \\
\text { Hallier f. }\end{array}$ & $\begin{array}{l}\text { Ridley (1900) as Ipomoea chryseides } \\
\text { Ker Gawl.; Keng (1990) }\end{array}$ \\
\hline Cyperaceae & $\begin{array}{l}\text { Cyperus leptocarpus (F.Muell.) } \\
\text { Bauters }\end{array}$ & $\begin{array}{l}\text { Ridley (1900) as Lipocarpha } \\
\text { microcephala (R.Br.) Kunth }\end{array}$ \\
\hline Cyperaceae & Diplacrum caricinum $R . B r$. & $\begin{array}{l}\text { Keng et al. (1998) as Scleria caricina } \\
\text { (R.Br.) Benth. }\end{array}$ \\
\hline Cyperaceae & Fimbristylis acuminata Vahl & Ridley (1900); Keng et al. (1998) \\
\hline Cyperaceae & $\begin{array}{l}\text { Fimbristylis dichotoma (L.) Vahl } \\
\text { subsp. dichotoma }\end{array}$ & $\begin{array}{l}\text { Ridley }(1900) \text { as Fimbristylis diphylla } \\
\text { var. pluristriata C.B.Clarke; Keng et al. } \\
\text { (1998) }\end{array}$ \\
\hline
\end{tabular}


Appendix II. Continuation.

\begin{tabular}{|c|c|c|}
\hline Family & Accepted Name & Reference \\
\hline Cyperaceae & Fimbristylis leptoclada Benth. & Ridley (1900); Keng et al. (1998) \\
\hline Cyperaceae & $\begin{array}{l}\text { Fimbristylis obtusata (C.B.Clarke) } \\
\text { Ridl. }\end{array}$ & $\begin{array}{l}\text { Ridley }(1900) \text { as Fimbristylis tenera } \\
\text { var. obtusata C.B.Clarke; Keng et al. } \\
\text { (1998) }\end{array}$ \\
\hline Cyperaceae & Fimbristylis pauciflora $\mathrm{R} . \mathrm{Br}$. & Keng et al. (1998) \\
\hline Cyperaceae & Fuirena umbellata Rottb. & Keng et al. (1998) \\
\hline Cyperaceae & Gahnia tristis Nees & Ridley (1900); Keng et al. (1998) \\
\hline Cyperaceae & Mapania longiflora C.B.Clarke & Keng et al. (1998) \\
\hline Cyperaceae & Scleria biflora Roxb. & Ridley (1900); Keng et al. (1998) \\
\hline Cyperaceae & Scleria corymbosa Roxb. & Keng et al. (1998) \\
\hline Cyperaceae & Scleria purpurascens Steud. & $\begin{array}{l}\text { Ridley (1900) as Scleria multifoliata } \\
\text { Boeckeler; Keng et al. (1998) }\end{array}$ \\
\hline Cyperaceae & Scleria sumatrensis Retz. & Keng et al. (1998) \\
\hline Cyperaceae & Scleria terrestris (L.) Fassett & $\begin{array}{l}\text { Ridley (1900) as Scleria radula Hance; } \\
\text { Keng et al. (1998) }\end{array}$ \\
\hline Dioscoreaceae & Dioscorea kingii R.Knuth & Ho et al. (2018) \\
\hline Dipterocarpaceae & Hopea ferruginea Parijs & Khoo et al. (2018) \\
\hline Dipterocarpaceae & $\begin{array}{l}\text { Vatica odorata (Griff.) Symington } \\
\text { subsp. odorata }\end{array}$ & Khoo et al. (2018) \\
\hline Elaeocarpaceae & $\begin{array}{l}\text { Elaeocarpus palembanicus (Miq.) } \\
\text { Corner }\end{array}$ & $\begin{array}{l}\text { Ridley (1900); Keng (1990), both as } \\
\text { Elaeocarpus hullettii King }\end{array}$ \\
\hline Euphorbiaceae & $\begin{array}{l}\text { Neoscortechinia philippinensis } \\
\text { (Merr.) Welzen }\end{array}$ & Khoo et al. (2018) \\
\hline Fabaceae & Intsia palembanica Miq. & Ali Ibrahim et al. (1997) \\
\hline Fabaceae & Sindora echinocalyx Prain & Khoo et al. (2018) \\
\hline Gesneriaceae & Aeschynanthus radicans Jack & Ridley (1900); Keng (1990) \\
\hline Hanguanaceae & $\begin{array}{l}\text { Hanguana corneri Škorničk. \& } \\
\text { P.C.Boyce }\end{array}$ & Leong-Škorničková \& Boyce (2015) \\
\hline
\end{tabular}


Appendix II. Continuation.

\begin{tabular}{|c|c|c|}
\hline Family & Accepted Name & Reference \\
\hline Hanguanaceae & $\begin{array}{l}\text { Hanguana neglecta Škorničk. \& } \\
\text { Niissalo }\end{array}$ & $\begin{array}{l}\text { Niissalo et al. (2014); Leong- } \\
\text { Škorničková \& Boyce (2015) }\end{array}$ \\
\hline Hanguanaceae & $\begin{array}{l}\text { Hanguana rubinea Škorničk. \& } \\
\text { P.C.Boyce }\end{array}$ & Leong-Škorničková \& Boyce (2015) \\
\hline Hanguanaceae & $\begin{array}{l}\text { Hanguana triangulata Škorničk. \& } \\
\text { P.C.Boyce }\end{array}$ & Leong-Škorničková \& Boyce (2015) \\
\hline Lamiaceae & Callicarpa longifolia Lam. & Ridley (1900); Keng (1990) \\
\hline Lamiaceae & Vitex negundo L. & Ridley (1900) \\
\hline Lauraceae & Cinnamomum javanicum Blume & Keng (1990) \\
\hline Lauraceae & $\begin{array}{l}\text { Cryptocarya nitens (Blume) Koord. } \\
\text { \& Valeton }\end{array}$ & de Kok (2015) \\
\hline Lauraceae & Dehaasia cuneata (Blume) Blume & Khoo et al. (2018) \\
\hline Lauraceae & Endiandra maingayi Hook.f. & Khoo et al. (2018) \\
\hline Lentibulariaceae & Utricularia aurea Lour. & $\begin{array}{l}\text { Ridley (1900) as Utricularia flexuosa } \\
\text { Vahl }\end{array}$ \\
\hline Linderniaceae & Lindernia crustacea (L.) F.Muell. & Keng (1990) \\
\hline Malpighiaceae & $\begin{array}{l}\text { Aspidopterys concava (Wall.) } \\
\text { A.Juss. }\end{array}$ & Keng (1990) \\
\hline Malvaceae & Sterculia cordata Blume & Keng (1990) \\
\hline Marantaceae & $\begin{array}{l}\text { Thaumatococcus daniellii (Benn.) } \\
\text { Benth. ex Eichler }\end{array}$ & Niissalo et al. (2016) \\
\hline Melastomataceae & $\begin{array}{l}\text { Diplectria divaricata (Willd.) } \\
\text { Kuntze }\end{array}$ & $\begin{array}{l}\text { Ridley (1900) as Anplectrum glaucum } \\
\text { (Jack) Triana }\end{array}$ \\
\hline Melastomataceae & $\begin{array}{l}\text { Memecylon acuminatum Sm. var. } \\
\text { acuminatum }\end{array}$ & $\begin{array}{l}\text { Ridley (1900) as Memecylon } \\
\text { acuminatum } \mathrm{Sm} .\end{array}$ \\
\hline Meliaceae & Aglaia crassinervia Kurz ex Hiern & Khoo et al. (2018) \\
\hline Meliaceae & Aglaia palembanica Miq. & Keng (1990); Ho et al. (2018) \\
\hline
\end{tabular}


Appendix II. Continuation.

\begin{tabular}{|c|c|c|}
\hline Family & Accepted Name & Reference \\
\hline Meliaceae & $\begin{array}{l}\text { Chisocheton pentandrus (Blanco) } \\
\text { Merr. subsp. paucijugus (Miq.) } \\
\text { Mabb. }\end{array}$ & Keng (1990) \\
\hline Meliaceae & Dysoxylum grande Hiern & Khoo et al. (2018) \\
\hline Meliaceae & Dysoxylum excelsum Blume & $\begin{array}{l}\text { Ridley (1900) as Dysoxylum turbinatum } \\
\text { King }\end{array}$ \\
\hline Moraceae & Antiaris toxicaria Lesch. & Keng (1990) \\
\hline Moraceae & Ficus heteropleura Blume & $\begin{array}{l}\text { Ridley (1900) as Ficus urophylla Wall. } \\
\text { ex Miq.; Keng (1990) }\end{array}$ \\
\hline Muntingiaceae & Muntingia calabura $\mathrm{L}$. & Ascher et al. (2019) \\
\hline Orchidaceae & Acriopsis ridleyi Hook.f. & Leong et al. (2018) \\
\hline Orchidaceae & Appendicula lucida Ridl. & Keng et al. (1998) \\
\hline Orchidaceae & Appendicula uncata Ridl. & Keng et al. (1998) \\
\hline Orchidaceae & Oberonia ciliolata Hook.f. & Ridley (1900) \\
\hline Orchidaceae & Peristylus lacertifer (Lind1.) J.J.Sm. & Keng et al. (1998) \\
\hline Orchidaceae & Pinalia floribunda (Lindl.) Kuntze & Leong et al. (2017) \\
\hline Orchidaceae & $\begin{array}{l}\text { Thrixspermum calceolus (Lindl.) } \\
\text { Rchb.f. }\end{array}$ & Ridley (1900) \\
\hline Passifloraceae & $\begin{array}{l}\text { Passiflora quadriglandulosa } \\
\text { Rodschied }\end{array}$ & Ho et al. (2018) \\
\hline Phyllanthaceae & Baccaurea minor Hook.f. & Ridley (1900); Keng (1990) \\
\hline Phyllanthaceae & Breynia discigera Müll.Arg. & Ridley (1900); Keng (1990) \\
\hline Phyllanthaceae & Phyllanthus reticulatus Poir. & Lim et al. (2018) \\
\hline Plantaginaceae & Adenosma inopinatum Prain & Keng (1990) \\
\hline Plantaginaceae & $\begin{array}{l}\text { Adenosma javanicum (Blume) } \\
\text { Koord. }\end{array}$ & Keng (1990) \\
\hline Plantaginaceae & Bacopa monnieri (L.) Pennell & $\begin{array}{l}\text { Ridley (1900) as Herpestes monniera } \\
\text { (L.) Kunth; Keng (1990) }\end{array}$ \\
\hline
\end{tabular}


Appendix II. Continuation.

\begin{tabular}{|c|c|c|}
\hline Family & Accepted Name & Reference \\
\hline Plantaginaceae & Limnophila villosa Blume & $\begin{array}{l}\text { Ridley (1900) as Limnophila } \\
\text { pulcherrima auct. non Hook.f.; Keng } \\
\text { (1990) }\end{array}$ \\
\hline Poaceae & $\begin{array}{l}\text { Cyrtococcum accrescens (Trin.) } \\
\text { Stapf }\end{array}$ & $\begin{array}{l}\text { Ridley (1900) as Panicum patens L.; } \\
\text { Keng et al. (1998) }\end{array}$ \\
\hline Poaceae & Echinochloa colona (L.) Link & Keng et al. (1998) \\
\hline Poaceae & Eragrostis brownii (Kunth) Nees & $\begin{array}{l}\text { Ridley (1900); Keng et al. (1998), both } \\
\text { as Eragrostis elongata (Willd.) J.Jacq. }\end{array}$ \\
\hline Poaceae & $\begin{array}{l}\text { Thysanolaena latifolia (Roxb. ex } \\
\text { Hornem.) Honda }\end{array}$ & Keng et al. (1998) \\
\hline Polypodiaceae & $\begin{array}{l}\text { Selliguea stenophylla (Blume) } \\
\text { Parris }\end{array}$ & $\begin{array}{l}\text { Ridley (1900) as Pleopeltis stenophylla } \\
\text { (Blume) T.Moore }\end{array}$ \\
\hline Putranjavaceae & $\begin{array}{l}\text { Drypetes crassipes Pax \& } \\
\text { K.Hoffm. }\end{array}$ & Khoo et al. (2018) \\
\hline Rhizophoraceae & Pellacalyx axillaris Korth. & Ridley (1900) \\
\hline Rosaceae & $\begin{array}{l}\text { Rubus moluccanus L. var. } \\
\text { moluccanus }\end{array}$ & Keng (1990) \\
\hline Rubiaceae & $\begin{array}{l}\text { Gaertnera obesa Hook.f. ex } \\
\text { C.B.Clarke }\end{array}$ & Ridley (1900) \\
\hline Rubiaceae & Gardeniopsis longifolia Miq. & Ridley (1900); Keng (1990) \\
\hline Rubiaceae & Lasianthus chryseus Ridl. & Keng (1990) \\
\hline Rubiaceae & Lasianthus ellipticus Wight & Ridley (1900) \\
\hline Rubiaceae & Psychotria deltata I.M.Turner & Turner \& Kumar (2018) \\
\hline Rutaceae & Luvunga crassifolia Tanaka & $\begin{array}{l}\text { Ridley (1900) as Luvunga } \\
\text { eleutherandra auct. non Dalzell }\end{array}$ \\
\hline Sapindaceae & $\begin{array}{l}\text { Lepisanthes fruticosa (Roxb.) } \\
\text { Leenh. }\end{array}$ & Khoo et al. (2018) \\
\hline Sapindaceae & $\begin{array}{l}\text { Nephelium cuspidatum Blume var. } \\
\text { eriopetalum (Miq.) Leenh. }\end{array}$ & $\begin{array}{l}\text { Ridley (1900) as Nephelium } \\
\text { eriopetalum Miq.; Keng (1990) as } \\
\text { Nephelium cuspidatum Blume }\end{array}$ \\
\hline
\end{tabular}


Appendix II. Continuation.

\begin{tabular}{|c|c|c|}
\hline Family & Accepted Name & Reference \\
\hline Sapotaceae & Palaquium impressionervium $\mathrm{Ng}$ & Khoo et al. (2018) \\
\hline Smilacaceae & Smilax megacarpa A.DC. & Ridley (1900) \\
\hline Tectariaceae & Tectaria nayarii Mazumdar & Ho et al. (2018) \\
\hline Thymelaeaceae & Gonystylus maingayi Hook.f. & Ridley (1901); Keng (1990) \\
\hline Urticaceae & Pouzolzia zeylanica (L.) Benn. & $\begin{array}{l}\text { Ridley (1900) as Pouzolzia indica } \\
\text { Gaudich.; Keng (1990) }\end{array}$ \\
\hline Vitaceae & $\begin{array}{l}\text { Ampelocissus cinnamomea (Wall.) } \\
\text { Planch. }\end{array}$ & Keng (1990); Ng et al. (2014) \\
\hline Zingiberaceae & Amomum xanthophlebium Baker & $\begin{array}{l}\text { Keng et al. (1998); Niissalo et al. } \\
\text { (2017) }\end{array}$ \\
\hline Zingiberaceae & $\begin{array}{l}\text { Etlingera maingayi (Baker) } \\
\text { R.M.Sm. }\end{array}$ & $\begin{array}{l}\text { Ridley (1900) as Hornstedtia maingayi } \\
\text { (Baker) Ridl.; Niissalo et al. (2017) }\end{array}$ \\
\hline Zingiberaceae & $\begin{array}{l}\text { Globba variabilis Ridl. subsp. } \\
\text { pusilla } \text { S.N.Lim }\end{array}$ & $\begin{array}{l}\text { Keng et al. (1998) as Globba variabilis } \\
\text { Ridl.; Niissalo et al. (2017) }\end{array}$ \\
\hline Zingiberaceae & Plagiostachys lateralis (Ridl.) Ridl. & $\begin{array}{l}\text { Ridley (1900); Keng et al. (1998); } \\
\text { Niissalo et al., (2017) }\end{array}$ \\
\hline Zingiberaceae & Scaphochlamys tenuis Holttum & Ali Ibrahim et al. (1997) \\
\hline Zingiberaceae & Zingiber singapurense Škorničk. & Niissalo et al. (2018) \\
\hline
\end{tabular}


Appendix III. List of species, along with their respective vouchers, newly recorded for BTNR in this study. Species are listed in alphabetical order of the families and then species. All specimens are deposited in SING unless otherwise stated.

\begin{tabular}{|c|c|c|}
\hline Family & Accepted Name & Voucher \\
\hline Acanthaceae & $\begin{array}{l}\text { Asystasia gangetica }(\mathrm{L} .) \text { T.Anderson } \\
\text { subsp. micrantha }(\mathrm{Nees}) \text { Ensermu }\end{array}$ & $\begin{array}{l}\text { BTLST51-08H; also observed at } \\
\text { LST48, LST49 \& LST52 }\end{array}$ \\
\hline Acanthaceae & $\begin{array}{l}\text { Strobilanthes reptans (G.Forst.) } \\
\text { Moylan ex Y.F.Deng \& J.R.I.Wood }\end{array}$ & BTMRP37-32H; BTLST51-07H \\
\hline Acanthaceae & Thunbergia fragrans Roxb. & BTLST48-06C \\
\hline Anacardiaceae & $\begin{array}{l}\text { Buchanania arborescens (Blume) } \\
\text { Blume }\end{array}$ & BTMRP39-32T; SING2005-56 \\
\hline Anacardiaceae & $\begin{array}{l}\text { Dracontomelon dao (Blanco) Merr. } \\
\& \text { Rolfe }\end{array}$ & BTTBS09-14T \\
\hline Anacardiaceae & Mangifera indica $\mathrm{L}$. & BTMRP38-08T; Tang \& Sidek 950 \\
\hline Anacardiaceae & $\begin{array}{l}\text { Melanochyla caesia (Blume) Ding } \\
\text { Hou }\end{array}$ & BTFVS45-11T; Tang \& Sidek 1003 \\
\hline Annonaceae & $\begin{array}{l}\text { Alphonsea maingayi Hook.f. \& } \\
\text { Thomson }\end{array}$ & $\begin{array}{l}\text { BT2015-052; BTTTS18-10T; } \\
\text { BTTTS19-45T; BTJFP22-23T; } \\
\text { SING2010-057; also observed at } \\
\text { MRP40 }\end{array}$ \\
\hline Annonaceae & Artabotrys costatus King & BTJFP26-37T \\
\hline Annonaceae & $\begin{array}{l}\text { Artabotrys maingayi Hook.f. \& } \\
\text { Thomson }\end{array}$ & BTCCP10-09T \\
\hline Annonaceae & Desmos chinensis Lour. & BTFVS43-13C \\
\hline Annonaceae & Desmos dumosus (Roxb.) Saff. & $\begin{array}{l}\text { BTMRP33-15C; BTMRP37-08C; } \\
\text { Ridley } 6305\end{array}$ \\
\hline Annonaceae & $\begin{array}{l}\text { Friesodielsia biglandulosa (Blume) } \\
\text { Steenis }\end{array}$ & BTTBS08-63C \\
\hline Annonaceae & $\begin{array}{l}\text { Friesodielsia latifolia (Hook.f. \& } \\
\text { Thomson) Steenis }\end{array}$ & BTTBS08-01C \\
\hline Annonaceae & $\begin{array}{l}\text { Maasia sumatrana (Miq.) Mols, } \\
\text { Kessler \& Rogstad }\end{array}$ & BTJFP24-21T; SING2010-082 \\
\hline Annonaceae & $\begin{array}{l}\text { Uvaria cuneifolia (Hook.f. \& } \\
\text { Thomson) L.L.Zhou, Y.C.F.Su \& } \\
\text { R.M.K.Saunders }\end{array}$ & BTJFP26-38C \\
\hline
\end{tabular}


Appendix III. Continuation.

\begin{tabular}{|c|c|c|}
\hline Family & Accepted Name & Voucher \\
\hline Annonaceae & Uvaria hirsuta Jack & BTJFP22-44C; SING2010-079 \\
\hline Annonaceae & Uvaria lobbiana Hook.f. \& Thomson & BTSVP02-10C \\
\hline Apocynaceae & Anodendron candolleanum Wight & $\begin{array}{l}\text { BTSVP05-51C; BTTBS08-12C; also } \\
\text { observed along JFP }\end{array}$ \\
\hline Apocynaceae & Hoya latifolia G.Don & $\begin{array}{l}\text { SING2016-111; also observed at } \\
\text { TTS21, JFS29, MRP37, FVS41 \& } \\
\text { FVS46 }\end{array}$ \\
\hline Apocynaceae & Strophanthus caudatus (L.) Kurz & BTMRP39-22C \\
\hline Apocynaceae & $\begin{array}{l}\text { Tabernaemontana corymbosa Roxb. } \\
\text { ex Wall. }\end{array}$ & $\begin{array}{l}\text { Tang \& Sidek 1027; also observed at } \\
\text { CCP17 }\end{array}$ \\
\hline Apocynaceae & $\begin{array}{l}\text { Urceola polyneura (Hook.f.) } \\
\text { D.J.Middleton \& Livsh. }\end{array}$ & $\begin{array}{l}\text { BTSVP04-28C; BTFVS41-41C; } \\
\text { BTTBS08-36T }\end{array}$ \\
\hline Aquifoliaceae & Ilex cymosa Blume & Kassim 293; also observed at JFP25 \\
\hline Araceae & $\begin{array}{l}\text { Dieffenbachia seguine (Jacq.) Schott } \\
\text { var. seguine }\end{array}$ & BTLST52-06H \\
\hline Araceae & $\begin{array}{l}\text { Epipremnum aureum (Linden ex } \\
\text { André) G.S.Bunting }\end{array}$ & BTLST52-08C \\
\hline Araceae & Lasia spinosa (L.) Thwaites & $\begin{array}{l}\text { BTLST50-09H; also observed at } \\
\text { LST51 \& LST52 }\end{array}$ \\
\hline Araceae & Rhaphidophora minor Hook.f. & BTMRP33-04C \\
\hline Araceae & Syngonium podophyllum Schott & $\begin{array}{l}\text { BTLST49-12C; also observed at } \\
\text { LST48 }\end{array}$ \\
\hline Arecaceae & Calamus ridleyanus Becc. & BT2016-036 \\
\hline Arecaceae & Elaeis guineensis Jacq. & $\begin{array}{l}\text { BTLST49-09T; also observed at } \\
\text { TTS20 \& LST48 }\end{array}$ \\
\hline Arecaceae & Eleiodoxa conferta (Griff.) Burret & BTTBS08-69S \\
\hline Arecaceae & Korthalsia flagellaris Miq. & BTMRP37-35C \\
\hline Arecaceae & $\begin{array}{l}\text { Nenga pumila (Blume) H.Wendl. var. } \\
\text { pachystachya (Blume) Fernando }\end{array}$ & BT2015-061 \\
\hline
\end{tabular}


Appendix III. Continuation.

\begin{tabular}{|c|c|c|}
\hline Family & Accepted Name & Voucher \\
\hline Arecaceae & $\begin{array}{l}\text { Plectocomia elongata Mart. ex } \\
\text { Blume }\end{array}$ & $\begin{array}{l}\text { BTSVP06-60C; also observed at } \\
\text { SVP05, TBS08 and along CCP }\end{array}$ \\
\hline Arecaceae & $\begin{array}{l}\text { Plectocomiopsis geminiflora (Griff.) } \\
\text { Becc. }\end{array}$ & BTSVP06-59C \\
\hline Arecaceae & $\begin{array}{l}\text { Ptychosperma macarthurii } \\
\text { (H.Wendl. ex H.J.Veitch) H.Wendl. } \\
\text { ex Hook.f. }\end{array}$ & BTLST48-13T \\
\hline Asparagaceae & Cordyline fruticosa (L.) A.Chev. & BTLST48-09S \\
\hline Asparagaceae & Dracaena elliptica Thunb. & $\begin{array}{l}\text { BTTTS18-28H; BTJFP26-43T; } \\
\text { Ridley } 3587 \text { (BM) }\end{array}$ \\
\hline Asparagaceae & Dracaena fragrans (L.) Ker Gawl. & $\begin{array}{l}\text { BTLST52-07T; also observed at } \\
\text { FVS47 }\end{array}$ \\
\hline Asparagaceae & Dracaena porteri Baker & $\begin{array}{l}\text { Ridley s.n., 1892; also observed at } \\
\text { JFS29 }\end{array}$ \\
\hline Asparagaceae & Dracaena sanderiana Mast. & BTLST48-10S \\
\hline Asteraceae & $\begin{array}{l}\text { Struchium sparganophorum (L.) } \\
\text { Kuntze }\end{array}$ & BTLST50-10H; Tang \& Sidek 919 \\
\hline Bignoniaceae & Spathodea campanulata P.Beauv. & ВТССР14-15T \\
\hline Calophyllaceae & $\begin{array}{l}\text { Calophyllum lanigerum Miq. var. } \\
\text { austrocoriaceum (Whitmore) } \\
\text { P.F.Stevens }\end{array}$ & BTTTS18-22T; Tang \& Sidek 1022 \\
\hline Calophyllaceae & Calophyllum macrocarpum Hook.f. & BTMRP34-07T; Khoo KMS81 \\
\hline Cannabaceae & Gironniera cf. hirta Ridl. & BT2015-067 \\
\hline Cannabaceae & Gironniera nervosa Planch. & $\begin{array}{l}\text { BTCCP10-46T; Mohd Noor MN431; } \\
\text { Mohd Noor MN759 }\end{array}$ \\
\hline Cardiopteridaceae & Gonocaryum gracile Miq. & BTCCP11-16T \\
\hline Celastraceae & Lophopetalum wightianum Arn. & BTFVS41-18T \\
\hline Celastraceae & Salacia maingayi M.A.Lawson & $\begin{array}{l}\text { BTJFP24-16C; also observed at } \\
\text { SVP04, TBS07 \& JFS29 }\end{array}$ \\
\hline
\end{tabular}


Appendix III. Continuation.

\begin{tabular}{|c|c|c|}
\hline Family & Accepted Name & Voucher \\
\hline Clusiaceae & $\begin{array}{l}\text { Garcinia atroviridis Griff. ex } \\
\text { T.Anderson }\end{array}$ & BTLST49-06T \\
\hline Clusiaceae & $\begin{array}{l}\text { Garcinia mangostana L. var. } \\
\text { malaccensis (Hook.f.) Nazre }\end{array}$ & BTFVS43-19T; Mohd Noor MN1529 \\
\hline Clusiaceae & Garcinia rostrata (Hassk.) Miq. & BTCCP16-10T; Hamzah s.n. \\
\hline Combretaceae & Combretum tetralophum C.B.Clarke & BT2016-039 \\
\hline Connaraceae & Connarus grandis Jack & $\begin{array}{l}\text { BTJFP25-08C; BTFVS45-15C; } \\
\text { SING2010-031 }\end{array}$ \\
\hline Connaraceae & Connarus monocarpus L. & $\begin{array}{l}\text { BTTTS19-17C; BTJFS29-07C; also } \\
\text { observed at SVP02 \& SVP06 }\end{array}$ \\
\hline Connaraceae & Connarus semidecandrus Jack & $\begin{array}{l}\text { BTSVP04-27C; BTLST50-01C; } \\
\text { Samsuri et al. EP16; also observed } \\
\text { along FVS, JFP, JFS, TBS \& TTS }\end{array}$ \\
\hline Connaraceae & $\begin{array}{l}\text { Rourea acutipetala Miq. subsp. } \\
\text { acutipetala }\end{array}$ & $\begin{array}{l}\text { BTSVP04-08C; BTJFP25-04C; also } \\
\text { observed at JFP23 \& JFS30 }\end{array}$ \\
\hline Connaraceae & $\begin{array}{l}\text { Rourea asplenifolia (G. Schellenb.) } \\
\text { Jongkind }\end{array}$ & BTCCP12-03C \\
\hline Connaraceae & Rourea mimosoides (Vahl) Planch. & BTFVS47-01C \\
\hline Convolvulaceae & Argyreia ridleyi (Prain) Ooststr. & BT2016-035 \\
\hline Cornaceae & Alangium frutescens Zoll. \& Moritzi & SING2016-179; BTFVS42-10C \\
\hline Cyperaceae & Hypolytrum nemorum (Vahl) Spreng. & $\begin{array}{l}\text { SING2019-102; Tang \& Sidek 1056; } \\
\text { also observed at TBS08 }\end{array}$ \\
\hline Cyperaceae & Scleria levis Retz. & BT2015-050 \\
\hline Dilleniaceae & Tetracera akara (Burm.f.) Merr. & BTTBS07-27C; BTCCP12-13C \\
\hline Dilleniaceae & Tetracera fagifolia Blume & $\begin{array}{l}\text { BTMRP40-20C; SING2010-028; also } \\
\text { observed at CCP10, JFP24, JFP25, } \\
\text { JFP26, JFS30, JFS32, LST50 \& } \\
\text { MRP39 }\end{array}$ \\
\hline Dilleniaceae & $\begin{array}{l}\text { Tetracera macrophylla Wall. ex } \\
\text { Hook.f. \& Thomson }\end{array}$ & $\begin{array}{l}\text { BTCCP16-01C; BTTTS20-22C; } \\
\text { BTFVS41-02C; SING2009-482; also } \\
\text { observed at TTS18, JFS28 \& JFS29 }\end{array}$ \\
\hline
\end{tabular}


Appendix III. Continuation.

\begin{tabular}{|c|c|c|}
\hline Family & Accepted Name & Voucher \\
\hline Dioscoreaceae & Dioscorea bulbifera $\mathrm{L}$. & BTTBS08-21C \\
\hline Dioscoreaceae & Dioscorea sansibarensis Pax & BTLST51-09C \\
\hline Dipterocarpaceae & Hopea sangal Korth. & $\begin{array}{l}\text { BT2016-009; BT2016-010; Khoo } \\
\text { KMS1; Khoo KMS61 }\end{array}$ \\
\hline Dipterocarpaceae & Shorea gibbosa Brandis & $\begin{array}{l}\text { BTTTS19-09T; BTTTS20-24T; Mohd } \\
\text { Nur } 3407\end{array}$ \\
\hline Ebenaceae & Diospyros confusa Bakh. & BTFVS46-01T; SING2010-008 \\
\hline Ebenaceae & Diospyros sumatrana Miq. & BTSVP05-08T; BTJFS32-10J \\
\hline Ebenaceae & Diospyros venosa Wall. ex A.DC. & $\begin{array}{l}\text { BTSVP03-15T; BTJFS32-11T; } \\
\text { SING2010-039; also observed at } \\
\text { SVP03, TTS19, JFP27, MRP34 \& } \\
\text { MRP36 }\end{array}$ \\
\hline Euphorbiaceae & Acalypha siamensis Oliv. ex Gage & BTMRP40-24S \\
\hline Euphorbiaceae & Cheilosa montana Blume & $\begin{array}{l}\text { BTFVS41-28T; Khoo et al. KMS58; } \\
\text { Corner SFN34985 }\end{array}$ \\
\hline Euphorbiaceae & $\begin{array}{l}\text { Claoxylon indicum (Reinw. ex } \\
\text { Blume) Hassk. }\end{array}$ & $\begin{array}{l}\text { BTLST48-11T; also observed at } \\
\text { SVP01 \& MRP33 [Ridley s.n., 1894, } \\
\text { reported by Turner \& Chua (2011) has } \\
\text { been redetermined as C. longifolium] }\end{array}$ \\
\hline Euphorbiaceae & $\begin{array}{l}\text { Hevea brasiliensis (Willd. ex } \\
\text { A.Juss.) Müll.Arg. }\end{array}$ & $\begin{array}{l}\text { BTLST50-08T; also observed at } \\
\text { JFP25, JFP26, JFP31 \& LST52 }\end{array}$ \\
\hline Euphorbiaceae & Macaranga hullettii King ex Hook.f. & $\begin{array}{l}\text { CCP15-32T; Hill H494; observed } \\
\text { along MRP }\end{array}$ \\
\hline Euphorbiaceae & Macaranga recurvata Gage & BTJFP25-10T; Ridley s.n., 1894 \\
\hline Fabaceae & $\begin{array}{l}\text { Archidendron jiringa }(\text { Jack }) \\
\text { I.C.Nielsen }\end{array}$ & $\begin{array}{l}\text { BTTBS09-02T; BTSVP01-10T; } \\
\text { BTMRP40-03J; Hill H523 }\end{array}$ \\
\hline Fabaceae & Callerya eriantha (Benth.) Schot & BTCCP10-07C \\
\hline Fabaceae & Dalbergia velutina Benth. & $\begin{array}{l}\text { BTCCP12-07C; also observed at } \\
\text { CCP16 \& JFP26 }\end{array}$ \\
\hline Fabaceae & Derris elliptica (Wall.) Benth. & BTLST52-01J \\
\hline
\end{tabular}


Appendix III. Continuation.

\begin{tabular}{|c|c|c|}
\hline Family & Accepted Name & Voucher \\
\hline Fabaceae & Spatholobus cf. ridleyi Prain & $\begin{array}{l}\text { BTTBS07-15C; BTJFP25-37C; also } \\
\text { observed at SVP04, TTS19, JFP24 \& } \\
\text { JFS32 }\end{array}$ \\
\hline Fagaceae & $\begin{array}{l}\text { Lithocarpus cf. gracilis (Korth.) } \\
\text { Soepadmo }\end{array}$ & BT2016-014 \\
\hline Flagellariaceae & Flagellaria indica $\mathrm{L}$. & BTSVP01-43C \\
\hline Gentianaceae & Cyrtophyllum fragrans (Roxb.) DC. & BTCCP12-30T \\
\hline Heliconiaceae & Heliconia psittacorum L.f. & BTLST52-09H; Tang \& Sidek 921 \\
\hline Hypericaceae & Cratoxylum maingayi Dyer & $\begin{array}{l}\text { Samsuri SA1323; also observed at } \\
\text { CCP15, JFP24 \& MRP39 }\end{array}$ \\
\hline Icacinaceae & Iodes cirrhosa Turcz. & BTFVS44-22C \\
\hline Icacinaceae & Iodes ovalis Blume & BTJFS29-15C; BTFVS47-08C \\
\hline Lauraceae & Actinodaphne pruinosa Nees & $\begin{array}{l}\text { BTJFS32-13T; also observed at } \\
\text { MRP35 \& MRP37 }\end{array}$ \\
\hline Lauraceae & $\begin{array}{l}\text { Alseodaphne nigrescens (Gamble) } \\
\text { Kosterm. }\end{array}$ & BTMRP34-29T \\
\hline Lauraceae & Cinnamomum iners Reinw. ex Blume & $\begin{array}{l}\text { BTLST48-08T; SING2011-247; also } \\
\text { observed along CCP, MRP, SVP \& } \\
\text { TBS plots }\end{array}$ \\
\hline Lauraceae & Cryptocarya cf. kurzii Hook.f. & BTJFP27-31T \\
\hline Lauraceae & Litsea costalis (Nees) Kosterm. & BTFVS43-21T; Mohd Noor MN1512 \\
\hline Lauraceae & Litsea robusta Blume & BTFVS45-21T \\
\hline Loganiaceae & Strychnos ignatii P.J.Bergius & BTSVP06-53C; SING2010-073 \\
\hline Lycopodiaceae & $\begin{array}{l}\text { Palhinhaea cernua (L.) Franco \& } \\
\text { Vasc. }\end{array}$ & $\begin{array}{l}\text { CCP12-29F; Liu SZ2002-77; Gurung } \\
7\end{array}$ \\
\hline Malpighiaceae & Hiptage sericea Hook.f. & $\begin{array}{l}\text { BTJFS30-34C; SING2012-470; } \\
\text { SING2014-200; SING2015-238; also } \\
\text { observed at SVP03 }\end{array}$ \\
\hline Malvaceae & Durio zibethinus L. & BTLST48-12T \\
\hline
\end{tabular}


Appendix III. Continuation.

\begin{tabular}{|c|c|c|}
\hline Family & Accepted Name & Voucher \\
\hline Malvaceae & Grewia laevigata Vahl & BTTBS08-68C \\
\hline Malvaceae & Sterculia parviflora Roxb. & BTSVP01-13T; BTTTS19-43T \\
\hline Melastomataceae & Memecylon cantleyi Ridl. & BTMRP39-19T \\
\hline Melastomataceae & Memecylon excelsum Blume & BTFVS41-47T \\
\hline Melastomataceae & Memecylon paniculatum Jack & BTJFS31-23T \\
\hline Meliaceae & Aglaia teysmanniana (Miq.) Miq. & $\begin{array}{l}\text { BTFVS45-18T; BTFVS45-22T; Khoo } \\
\text { KMS8 }\end{array}$ \\
\hline Meliaceae & $\begin{array}{l}\text { Chisocheton sarawakanus (C.DC.) } \\
\text { Harms }\end{array}$ & $\begin{array}{l}\text { BTFVS43-30T; Khoo \& Nik Faizu } \\
\text { KMS35 }\end{array}$ \\
\hline Menispermaceae & Tinospora macrocarpa Diels & BTSVP01-33C \\
\hline Moraceae & Artocarpus anisophyllus Miq. & BTTTS19-47T \\
\hline Moraceae & Artocarpus heterophyllus Lam. & BTFVS47-22T \\
\hline Moraceae & $\begin{array}{l}\text { Artocarpus nitidus Trécul subsp. } \\
\text { griffithii (King) F.M.Jarrett }\end{array}$ & BTTBS08-60T \\
\hline Moraceae & Ficus apiocarpa Miq. & $\begin{array}{l}\text { SING2010-746; also observed at } \\
\text { TBS09 }\end{array}$ \\
\hline Moraceae & Ficus pumila $\mathrm{L}$. & Ming A150; also observed at MRP40 \\
\hline Myristicaceae & Horsfieldia grandis (Hook.f.) Warb. & BTJFP26-25T \\
\hline Myristicaceae & Knema malayana Warb. & $\begin{array}{l}\text { BTJFP26-32T; also observed at } \\
\text { CCP14 \& JFP27 }\end{array}$ \\
\hline Myristicaceae & $\begin{array}{l}\text { Knema cf. sumatrana (Blume) } \\
\text { W.J.de Wilde }\end{array}$ & BTSVP04-11T \\
\hline Myristicaceae & Myristica crassa King & BTSVP05-53T; Tang \& Sidek 1041 \\
\hline Myristicaceae & Myristica iners Blume & BTSVP04-20T; BTSVP04-35T \\
\hline Myrtaceae & $\begin{array}{l}\text { Syzygium acuminatissimum (Blume) } \\
\text { DC. }\end{array}$ & BT2015-060; Hill H547; Ming A155 \\
\hline
\end{tabular}


Appendix III. Continuation.

\begin{tabular}{|c|c|c|}
\hline Family & Accepted Name & Voucher \\
\hline Myrtaceae & $\begin{array}{l}\text { Syzygium cf. glabratum (DC.) } \\
\text { Veldkamp }\end{array}$ & BTJFS30-05T \\
\hline Myrtaceae & $\begin{array}{l}\text { Syzygium claviflorum (Roxb.) Wall. } \\
\text { ex A.M.Cowan \& Cowan var. } \\
\text { claviflorum }\end{array}$ & $\begin{array}{l}\text { BTSVP05-16T; BTCCP13-35T; } \\
\text { BTMRP39-13J; also observed at } \\
\text { TBS07 \& TBS09 }\end{array}$ \\
\hline Myrtaceae & Syzygium pustulatum (Duthie) Merr. & $\begin{array}{l}\text { BTJFS28-18T; BTMRP40-08J; also } \\
\text { observed at TBS09, FVS46 and along } \\
\text { MRP; Mohd Noor MN1258 }\end{array}$ \\
\hline Myrtaceae & $\begin{array}{l}\text { Syzygium singaporense (King) Airy } \\
\text { Shaw }\end{array}$ & $\begin{array}{l}\text { Ngadiman SFN36355; also observed } \\
\text { at JFS31 }\end{array}$ \\
\hline Myrtaceae & $\begin{array}{l}\text { Syzygium syzygioides (Miq.) Merr. \& } \\
\text { L.M.Perry }\end{array}$ & $\begin{array}{l}\text { BTMRP35-06J; BTMRP37-31T; also } \\
\text { observed at LST51 \& LST52 }\end{array}$ \\
\hline Olacaceae & Erythropalum scandens Blume & BTFVS43-41C; at observed at TBS09 \\
\hline Oleaceae & Olea brachiata (Lour.) Merr. & BTSVP05-40T; BTJFS31-12T \\
\hline Opiliaceae & $\begin{array}{l}\text { Champereia manillana (Blume) } \\
\text { Merr. }\end{array}$ & BTSVP06-12T \\
\hline Oxalidaceae & Averrhoa carambola $\mathrm{L}$. & BTLST49-13T \\
\hline Pandanaceae & $\begin{array}{l}\text { Freycinetia sumatrana Hemsl. var. } \\
\text { sumatrana }\end{array}$ & $\begin{array}{l}\text { SING2019-101; also observed at } \\
\text { TBS08, TBS09, JFS29 \& JFS31 }\end{array}$ \\
\hline Pandanaceae & Pandanus amaryllifolius Roxb. & BTLST49-10S \\
\hline Phyllanthaceae & Aporosa confusa Gage & $\begin{array}{l}\text { BTFVS42-20T; BTFVS46-15S; } \\
\text { Ridley } 6486\end{array}$ \\
\hline Phyllanthaceae & $\begin{array}{l}\text { Baccaurea motleyana (Müll.Arg.) } \\
\text { Müll.Arg. }\end{array}$ & BTSVP01-38T \\
\hline Phyllanthaceae & Glochidion singaporense Gage & BTJFS30-16T \\
\hline Piperaceae & Piper neesii (Miq.) P.K.Mukh. & $\begin{array}{l}\text { BTLST49-11C; also observed at } \\
\text { TSB08, MRP34 \& FVS41 }\end{array}$ \\
\hline Piperaceae & Piper sarmentosum Roxb. & $\begin{array}{l}\text { BTLST52-10H; also observed at } \\
\text { SVP01 }\end{array}$ \\
\hline Poaceae & $\begin{array}{l}\text { Gigantochloa hasskarliana (Kurz) } \\
\text { Backer ex K.Heyne }\end{array}$ & BTFVS47-15B \\
\hline
\end{tabular}


Appendix III. Continuation.

\begin{tabular}{|c|c|c|}
\hline Family & Accepted Name & Voucher \\
\hline Poaceae & Ottochloa nodosa (Kunth) Dandy & BTLST48-05H; Duistermaat S85 \\
\hline Polygalaceae & Xanthophyllum discolor Chodat & BTJFS29-10T; BTMRP37-24T \\
\hline Polygalaceae & Xanthophyllum ellipticum Korth. & BTCCP13-07T; Mohd Noor MN1059 \\
\hline Polygalaceae & $\begin{array}{l}\text { Xanthophyllum griffithii Hook.f. ex } \\
\text { A.W.Benn. subsp. erectum Meijden }\end{array}$ & $\begin{array}{l}\text { Mohd Noor MN1386; also observed } \\
\text { at TBS09 [Mohd Shah \& Samsuri } \\
\text { MS3956 reported by Turner \& Chua } \\
\text { (2011) has been redetermined as } X \text {. } \\
\text { eurhynchum] }\end{array}$ \\
\hline Primulaceae & Ardisia ridleyi King \& Gamble & BTJFS29-20T \\
\hline Rhamnaceae & Smythea lanceata Summerh. & BT2015-065 \\
\hline Rubiaceae & Aidia auriculata (Wall.) Ridsdale & $\begin{array}{l}\text { BTMRP36-28C; also observed at } \\
\text { FVS47 }\end{array}$ \\
\hline Rubiaceae & Coptosapelta flavescens Korth. & BTFVS41-06C; SING2009-504 \\
\hline Rubiaceae & $\begin{array}{l}\text { Gardenia subcarinata (Corner) } \\
\text { Y.W.Low var. subcarinata }\end{array}$ & $\begin{array}{l}\text { BT2015-058; BTJFS32-05T; } \\
\text { BTJFP24-36T; SING2010-055; } \\
\text { Ngadiman SFN34926 }\end{array}$ \\
\hline Rubiaceae & $\begin{array}{l}\text { Gynochthodes rigida (Miq.) Razafim. } \\
\& \text { B.Bremer }\end{array}$ & $\begin{array}{l}\text { BTTBS09-41C; also observed at } \\
\text { TBS08 \& CCP11 }\end{array}$ \\
\hline Rubiaceae & Hedyotis verticillata (L.) Lam. & BTLST48-03H \\
\hline Rubiaceae & Psychotria sarmentosa Blume & BTCCP11-15C; Tang \& Sidek 924 \\
\hline Rubiaceae & Psydrax nitida (Craib) K.M.Wong & BTFVS41-23T \\
\hline Rutaceae & Clausena excavata Burm.f. & $\begin{array}{l}\text { BTLST49-08S; also observed at } \\
\text { SVP01, SVP06, FVS47, LST48 \& } \\
\text { LST51 }\end{array}$ \\
\hline Sapindaceae & $\begin{array}{l}\text { Dimocarpus longan Lour. var. } \\
\text { malesianus Leenh. }\end{array}$ & BTLST49-07T; Ridley 4782 \\
\hline Sapindaceae & $\begin{array}{l}\text { Lepisanthes rubiginosa (Roxb.) } \\
\text { Leenh. }\end{array}$ & BTFVS41-05T \\
\hline Sapindaceae & Mischocarpus sundaicus Blume & BTJFS30-01T \\
\hline
\end{tabular}


Appendix III. Continuation.

\begin{tabular}{lll}
\hline Family & Accepted Name & Voucher \\
\hline Sapindaceae & Nephelium laurinum Blume & $\begin{array}{l}\text { BTJFS29-24T; also observed at } \\
\text { FVS41 }\end{array}$ \\
Sapotaceae & Palaquium oxleyanum Pierre & $\begin{array}{l}\text { BTSVP02-19T, BTSVP04-14T; } \\
\text { Sinclair SFN40036 }\end{array}$ \\
Simaroubaceae & Brucea javanica (L.) Merr. & BTTBS09-30T \\
Smilacaceae & Smilax myosotiflora A.DC. & $\begin{array}{l}\text { BTJFP26-49C; also observed at } \\
\text { MRP38; Hill H557/1 }\end{array}$ \\
Symplocaceae & $\begin{array}{l}\text { Symplocos adenophylla } \text { Wall. ex } \\
\text { G.Don }\end{array}$ & $\begin{array}{l}\text { BTLST51-04T; also observed at } \\
\text { LST51 }\end{array}$ \\
Theaceae & Gordonia penangensis Ridl. & BTJFP24-40T \\
Vitaceae & Cayratia mollissima (Wall.) Gagnep. & MRP35-17C; Hardial 643 \\
Vitaceae & Cissus repens Lam. & BTFVS45-30C \\
Vitaceae & Pterisanthes cissioides Blume & BT2015-035 \\
Vitaceae & Tetrastigma dichotomum Planch. & $\begin{array}{l}\text { BTFVS41-33C; BTFVS47-13C; } \\
\text { SING2012-185 }\end{array}$ \\
& & Leong-Škorničková et al. SING27 \\
& &
\end{tabular}

